Prepared for the U.S. Department of Energy

under Contract DE-AC05-76RL01830

\title{
Transport Test Problems for Radiation Detection Scenarios
}

\author{
MW Shaver \\ EA Miller \\ RS Wittman \\ BS McDonald
}

September 2012

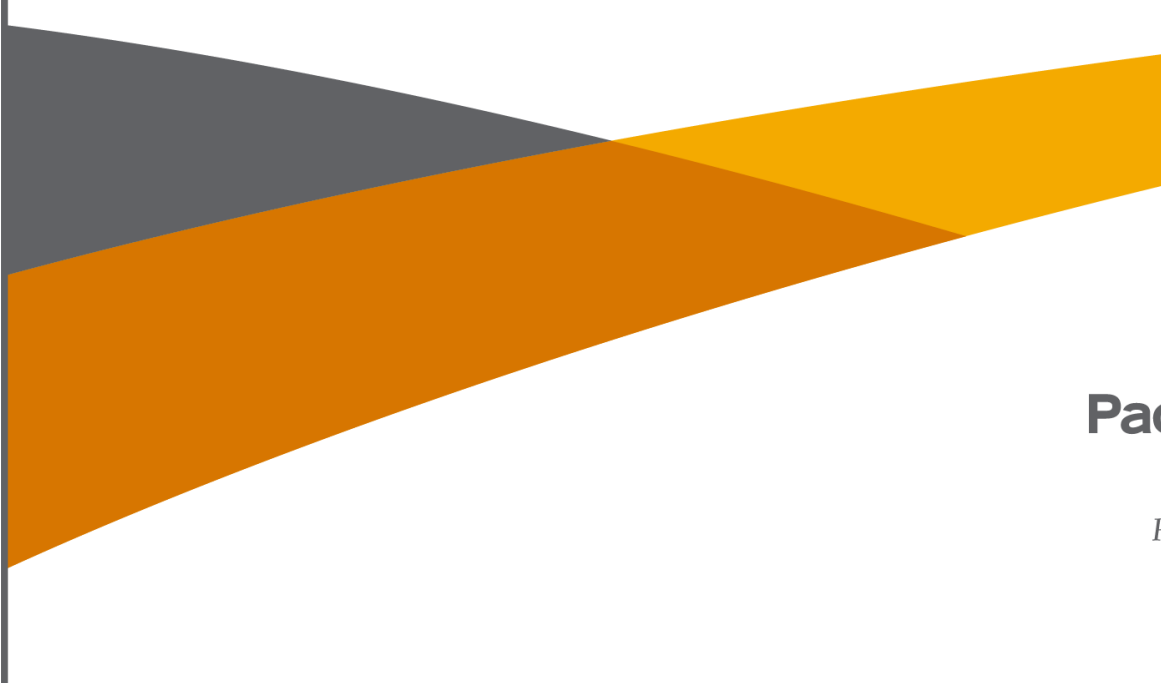




\title{
DISCLAIMER
}

This report was prepared as an account of work sponsored by an agency of the United States Government. Neither the United States Government nor any agency thereof, nor Battelle Memorial Institute, nor any of their employees, makes any warranty, express or implied, or assumes any legal liability or responsibility for the accuracy, completeness, or usefulness of any information, apparatus, product, or process disclosed, or represents that its use would not infringe privately owned rights. Reference herein to any specific commercial product, process, or service by trade name, trademark, manufacturer, or otherwise does not necessarily constitute or imply its endorsement, recommendation, or favoring by the United States Government or any agency thereof, or Battelle Memorial Institute. The views and opinions of authors expressed herein do not necessarily state or reflect those of the United States Government or any agency thereof.

\author{
PACIFIC NORTHWEST NATIONAL LABORATORY \\ operated by \\ BATTELLE \\ for the \\ UNITED STATES DEPARTMENT OF ENERGY \\ under Contract DE-AC05-76RL01830
}

Printed in the United States of America
Available to DOE and DOE contractors from the Office of Scientific and Technical Information,
P.O. Box 62, Oak Ridge, TN 37831-0062;
ph: (865) 576-8401
fax: $(865)$ 576-5728
email: reports@adonis.osti.gov

\begin{abstract}
Available to the public from the National Technical Information Service, U.S. Department of Commerce, 5285 Port Royal Rd., Springfield, VA 22161 ph: (800) 553-6847 fax: $(703) 605-6900$ email: orders@ntis.fedworld.gov online ordering: http://www.ntis.gov/ordering.htm
\end{abstract}

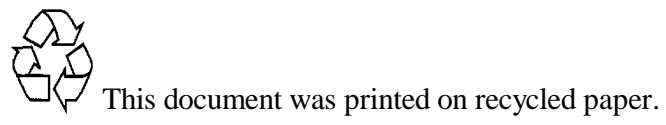




\section{Transport Test Problems for Radiation Detection Scenarios}

MW Shaver

EA Miller

RS Wittman

BS McDonald

September 2012

Prepared for

the U.S. Department of Energy

under Contract DE-AC05-76RL01830

Pacific Northwest National Laboratory

Richland, Washington 99352 



\section{Summary}

Modeling forms an important component of radiation detection development, allowing for testing of new detector designs, evaluation of existing equipment against a wide variety of potential threat sources, and assessing operation performance of radiation detection systems. This can, however, result in large and complex scenarios which are time consuming to model. A variety of approaches to radiation transport modeling exist with complementary strengths and weaknesses for different problems. This variety of approaches, and the development of promising new tools (such as ORNL's ADVANTG) which combine benefits of multiple approaches, illustrates the need for a means of evaluating or comparing different techniques for radiation detection problems.

This report presents a set of 9 test problems for comparing different types of radiation transport calculations, identifying appropriate tools for classes of problems, and testing and guiding the development of new methods. Cases are drawn primarily from existing or previous calculations with a preference for cases which include experimental data, or otherwise have results with a high level of confidence, are non-sensitive, and represent problem sets of interest to NA-22. From a technical perspective, cases are chosen to span a range of difficulty and to include gamma transport, neutron transport, or both and represent different important physical processes and a range of sensitivity to angular or energy fidelity.

Following test case identification, existing information about geometry, measurements, and previous calculations were assembled. Monte Carlo results (MCNP decks) were reviewed or created and re-run in order to attain accurate computational times and to verify agreement with experimental data, when present. Test case information was then conveyed to ORNL in order to guide testing and development of hybrid calculations. The results of those ADVANTG calculations were then sent to PNNL for compilation. This is a report describing the details of the selected test cases and results from available transport codes. Additional results from other transport codes will be added during the next year, along with additional cases if needed for software testing. Future work will extend the test problems to include more extensive crosscode comparisons as well as more refined metrics for evaluating the tradeoffs between operator time, computational time, and solution accuracy, to assemble a formalized benchmark set. 



\section{Acknowledgments}

The test problems summarized in this report represent a substantial effort in both radiation detection methods and transport model development by a number of staff from both PNNL and elsewhere. While the original work is referenced in this report, the original authors of the models and other contributors are thanked for sharing additional details such as MCNP input decks and for helpful direction in understanding and rerunning (or reproducing) their results. This list of authors of these input decks include includes Edward Siciliano, John Schweppe, Ben McDonald, Kim Burns, Sam Briggs, Mark Shaver, Rick Wittman, Andy Casella. Also, Mitch Woodring is thanked for sharing and clarifying his prototype radiation portal data. Gus Caffrey from INL is also thanked for providing models for the cask scanner and funding and direction for the RADSAT n, $y$ project that developed that case. Lastly, the authors would like to thank our ORNL collaborators (specifically Jeff Johnson and Scott Mosher), for guidance on useful test cases as well as information on the hybrid method they are developing. 



\section{Definitions, Acronyms, and Abbreviations}

ADVANTG $\underline{\text { Automate }} \underline{\mathbf{d}}$ Variance Reduction Generator - a Monte Carlo/Deterministic Hybrid transport code developed by ORNL, using Denovo, and MCNP

Attila A deterministic transport code developed by Transpire Inc.

CADIS $\quad \underline{\text { Consistent }} \underline{\text { Adjoint }}$ Driven Importance Sampling - a method for generating variance reduction parameters to accelerate the estimation of single-bin tallies

Denovo A 3-D, block-parallel discrete ordinates transport code developed at ORNL

Determinisitic In this document, refers to methods and codes that discretize the independent variables of the transport equation to construct linear algebraic systems of equations that are solved via iterative methods. This class of methods includes the discrete ordinates approximation.

FOM $\quad$ Figure of Merit - defined as $1 / R^{2} T$, where $\mathrm{R}$ is the tally relative error and $\mathrm{T}$ is the computational time

FW-CADIS Forward-Weighted CADIS - a method for generating variance reduction parameters to obtain relatively uniform statistical uncertainties across multiple tallies or space- and/or energy-dependent mesh tallies

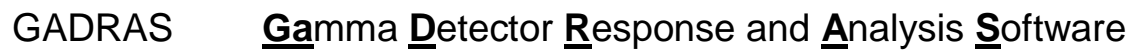

INL Idaho National Laboratory

LANL $\quad$ Los $\underline{\text { Alamos National Laboratories }}$

MCNP $\quad \underline{\text { Monte }} \underline{\text { Carlo }} \underline{\mathbf{N}}$-Particle - a continuous-energy neutron, photon, and electron transport code developed at Los Alamos National Laboratory.

Monte Carlo Methods and codes that simulate particle transport by sampling the individual events in particle's life history (e.g., emission by a source, free-streaming between collisions, collision, production of secondary particles, etc.)

ORNL $\quad$ Oak Ridge National Laboratory

PANDA Passive Nondestructive Assay of Nuclear Materials - NUREG/CR-5550/LA-UR90-732 written by LANL.

PNNL $\quad$ Pacific Northwest National Laboratory

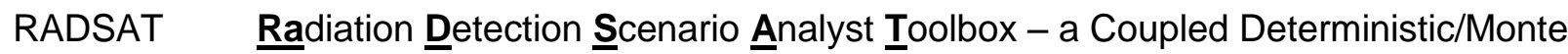
Carlo transport code developed by PNNL, where the bulk transport is done in Attila and the detector response is calculated in MCNP

VR $\quad$ Variance $\underline{R}$ eduction - procedures used to increase the precision of the estimates that can be obtained for a given number of iterations 



\section{Table of Contents}

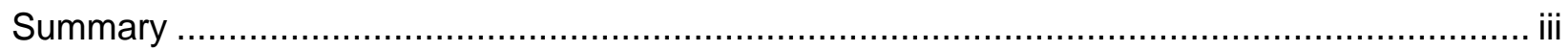

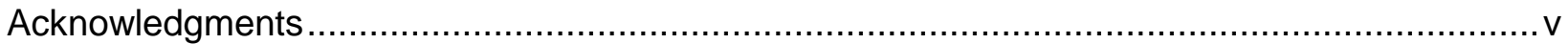

Definitions, Acronyms, and Abbreviations …................................................................ vii

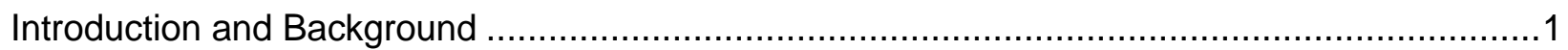

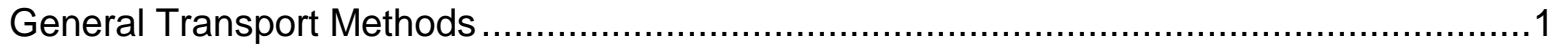

Stochastic/Monte Carlo Transport Methods.............................................. 1

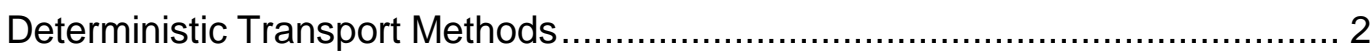

Coupled Transport Methods ..................................................................... 3

Hybrid Transport Methods ................................................................ 4

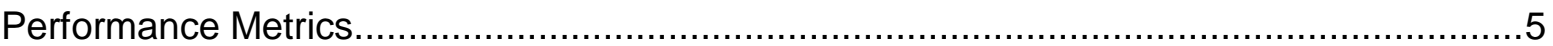

Code Quality (Solution Accuracy) ….................................................... 5

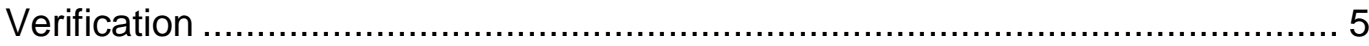

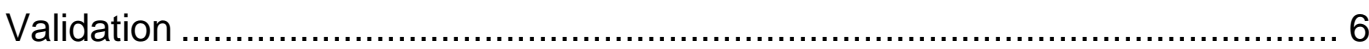

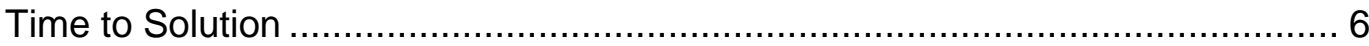

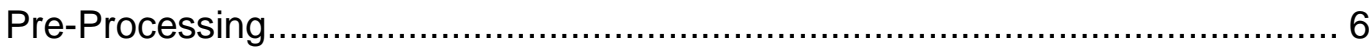

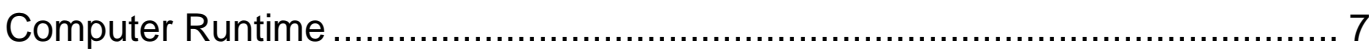

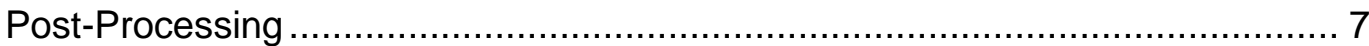

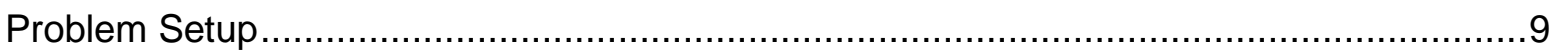

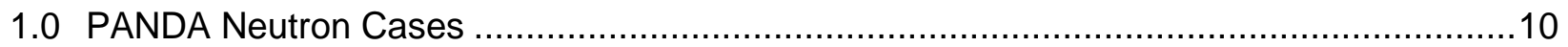

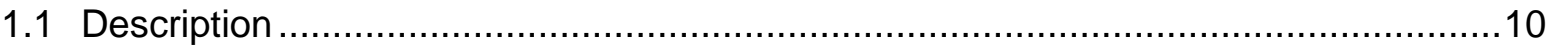

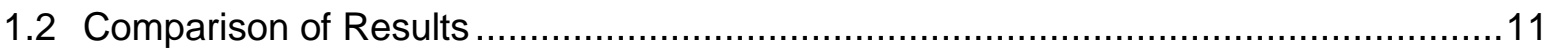

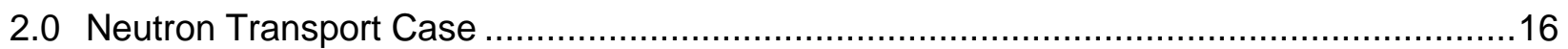

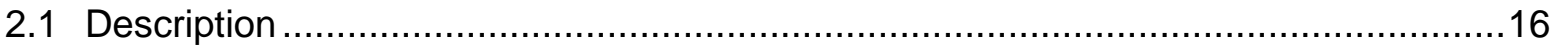

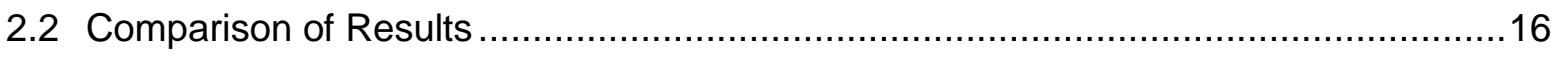

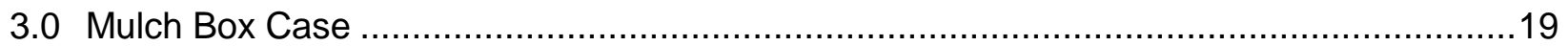

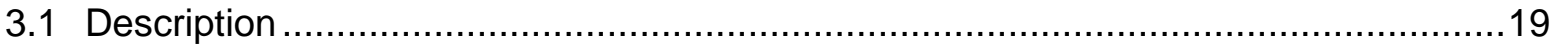

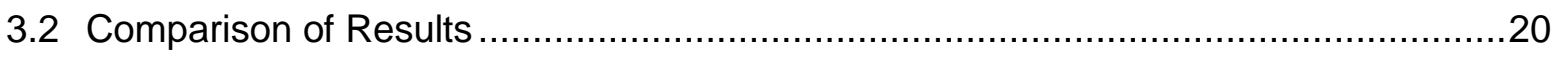

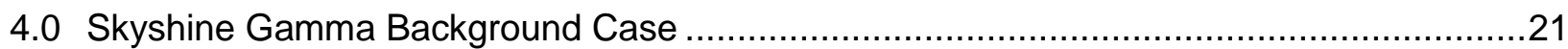

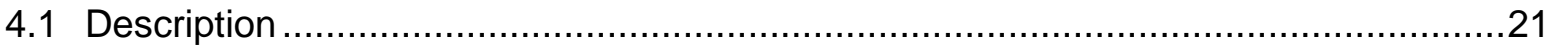

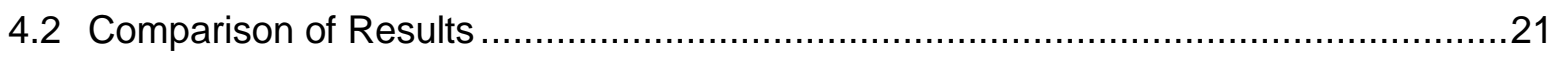

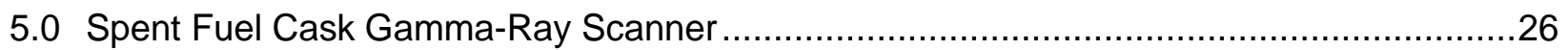

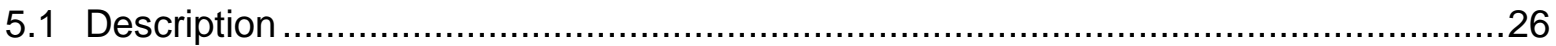

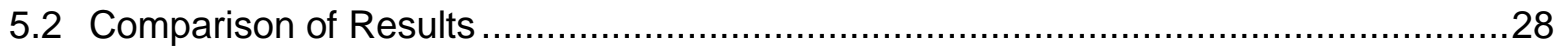

6.0 Generic Radiation Portal Monitor Case .......................................................................33

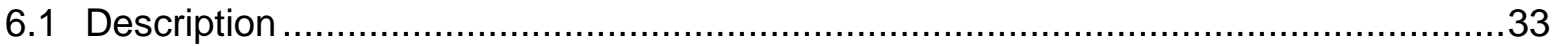

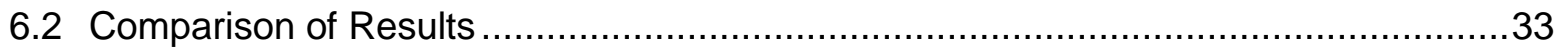

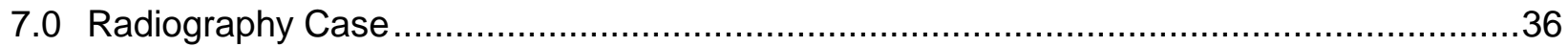




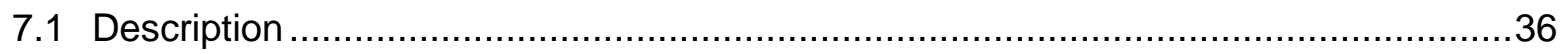

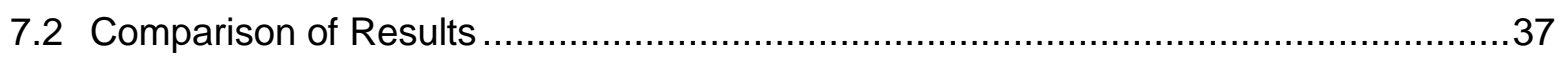

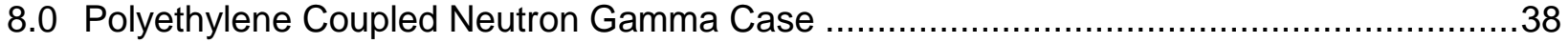

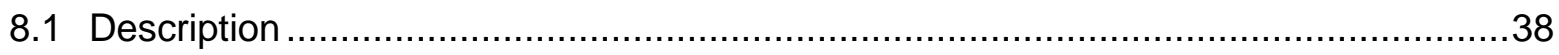

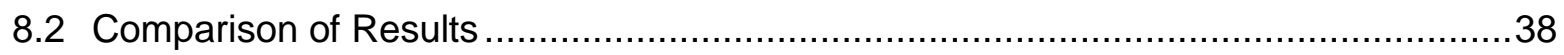

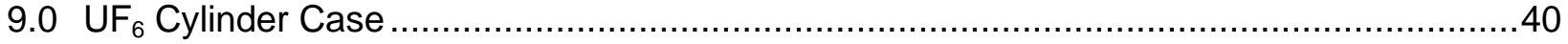

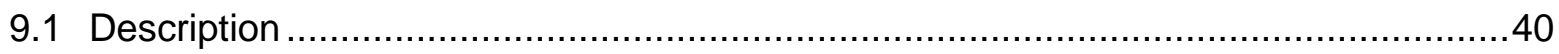

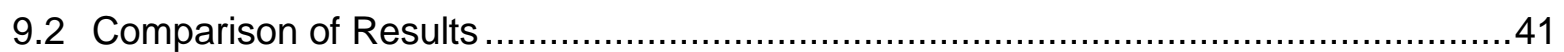

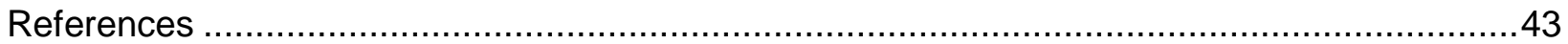




\section{List of Figures}

Figure 1. The geometry for the 14.4.1 PANDA case. [Stewart 1991] ..................................10

Figure 22. The geometry for the 14.4.2 PANDA case. [Stewart 1991] .................................10

Figure 3. The Attila geometry approximations for the 14.4.1 PANDA case. [Stewart

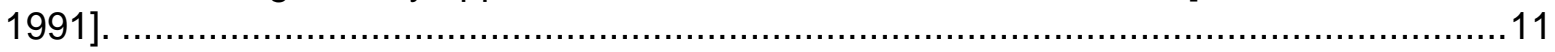

Figure 4. Results for the 14.4.1 PANDA case. [Stewart 1991] .........................................12

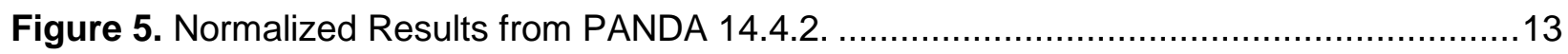

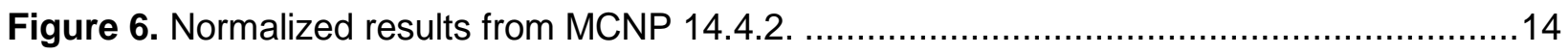

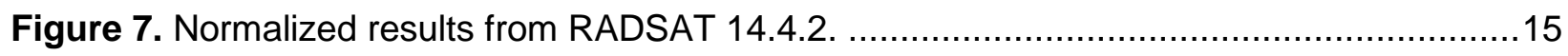

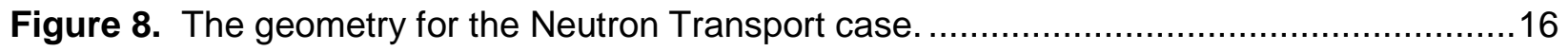

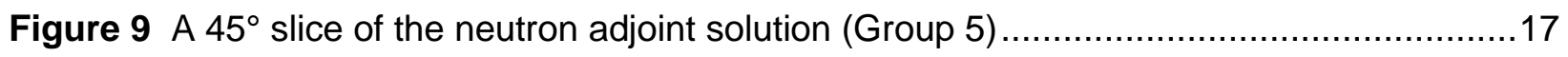

Figure 10 Runtime Comparisons for varying shielding thicknesses. ....................................18

Figure 11. The geometry for the Mulch Box case with a detailed cutout of the MCNP model of the detector in the upper right hand corner..............................................19

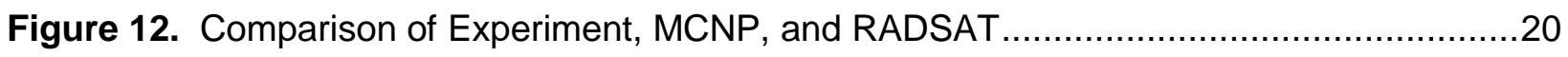

Figure 13. The geometry for the Skyshine Gamma Background Case. .................................21

Figure 142. Configuration Used in MCNP to Calculate the Hemispherical Surface

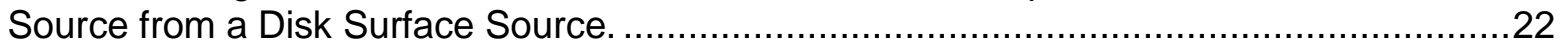

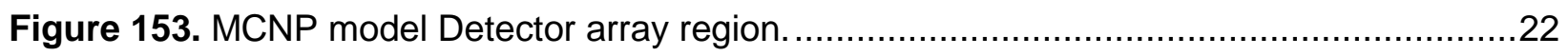

Figure 16. Comparison of the Calculated to Measured Gamma-Ray Background...................23

Figure 17. General tetrahedral mesh elements for air and ground......................................24

Figure 18. Single Detector used for Detector Response Function. ......................................24

Figure 19. Results for Small field (red) and Large field (black) Attila Model in RADSAT..........25

Figure 20. The geometry for the Spent Fuel Cask Gamma-Ray Scanner case [Caffrey 2009]. .26

Figure 21. Scanning Pattern of a Westinghouse MC-10 Spent Fuel Cask [Caffrey 2009]........26

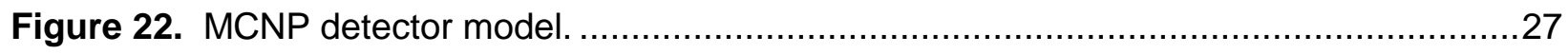

Figure 23. Configuration and loading of the Turkey Point spent fuel cask [Caffrey 2009]. ......27

Figure 24. MCNP Gamma-Ray Spent Fuel Cask Scanner Model showing region of lowest (blue) to highest (red) importance used for variance reduction..............................28

Figure 25. Solid Works CAD model of the spent fuel in the cask used for the Attila

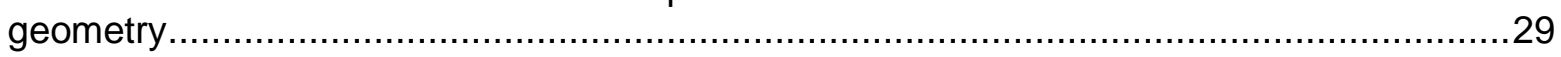

Figure 26. Comparison of Cask Scanner Detector Response in the full C2 slot......................29

Figure 27. Comparison of Cask Scanner Detector Response in the empty A4 slot...................30

Figure 28. Comparison of Cask Scanner Detector Response in the full C2 and empty A4 slots

Figure 29. The geometry for the Generic Radiation Portal Monitor case

Figure 30. Comparison of MCNP with ${ }^{60} \mathrm{Co}$ source measurements for data used as calibration. 
Figure 31. Comparison of MCNP with ${ }^{137} \mathrm{Cs}$ source measurements. ................................35

Figure 32. The geometry for the Radiography case ................................................. 36

Figure 33. Measured and simulated response for the $17.8 \mathrm{~cm}$-thick case. ..........................37

Figure 34. The geometry for the Polyethylene Coupled Neutron Gamma Case......................38

Figure 35. MCNP5 Results for Pulse-height Tally in HPGe Spectrometer ..............................39

Figure 36. The geometry for the $\mathrm{UF}_{6}$ cylinder case (MCNP model geometry on the right).......40

Figure 37. MCNP detector model geometry; (red) steel, (pink) poly and (green) Nal..............40

Figure 38. MCNP calculated detector response results. ................................................41

Figure 39. MCNP modeled versus measured data for a 4\% UF6 30B cylinder, with residuals plot on the bottom. 


\section{Introduction and Background}

In the last two decades, many new radiation detector designs have been developed for varied, non-traditional uses, specifically for national security related applications and scenarios. Since these systems and scenarios are new and different from the existing, traditional ones, little experimental data has been gathered. Experimental data may also be impossible to acquire in the case of analyzing conceptual designs, because they do not exist yet. As this new radiation detection equipment is built and deployed, the ability to predict the efficacy of these systems becomes very important. Of course, efficacy is best predicted through experimentation, but it is not practical to perform all such measurements because of time and cost constraints, as well as the availability of suitable threat sources. Many times, full prototypes are too expensive to build and test, so operational data is not always available, but some assurances are required before building them. For these scenarios, the best way to get the needed data is to model the detector systems with reliable and established computational tools.

Along with this trend of new applications of radiation detection, the scenario analysts continually perform larger calculations than ever before as computational capacity increases with microchip efficiency according to Moore's law. For example, methods developers have greatly improved variance reduction techniques and parallel processing of codes. With this new dependence on large-scale computing for detector development and analysis of detection scenarios comes a need for even larger calculations, thus increasing the importance of developing new simulation algorithms and methods.

\section{General Transport Methods}

For particle transport, there are two general categories of computational tools: stochastic and deterministic. Essentially, deterministic methods give exact results to an approximation (discretization) of the system, while Monte Carlo methods give approximate (statistical) results to an exact representation of the system, while the physics (cross sections) are essentially the same. Monte Carlo methods are typically the tool of choice for simulating the response of neutron and gamma-ray detectors operating in national security settings, but deterministic codes offer potential advantages in computational efficiency for many complex 3-D scenarios, particularly those with a high degree of attenuation or scattering. Because pulse-height tallies are inherently stochastic, Monte Carlo simulation of gamma-ray spectrometer response is straightforward. Pulse-height tallies are not an obvious extension of deterministic methods, so gamma-ray detection for deterministic methods is problematic. The idea has been around for a long time to combine the two in some way to gain the benefits of both, while trying to get around the limitations of both [Bell et al. 1970, Duderstadt et al. 1976, Lamarsh 1983, Parringron et al. 1996].

\section{Stochastic/Monte Carlo Transport Methods}

A common stochastic neutron transport code is Monte Carlo N-Particle (MCNP), which is developed at LANL. Of course, there are other codes that use the Monte Carlo methods (e.g. GEANT), but MCNP is the most prevalent and is the code used in these test cases to represent Monte Carlo methods. The Monte Carlo method is originally developed to help model particle transport for the Manhattan project in the first half of the twentieth century. The method is given 
its name because it resembled games of chance. It models representative particles for the duration of their lifetime using probability distribution functions at every interaction with the background medium [X-5 et al. 2001, Waters et al. 2002].

Monte Carlo codes continue to be used more frequently as computing speeds increases, due to their geometric flexibility, well-tested cross section libraries, and high accuracy through the direct simulation of fundamental physics processes. Variance reduction techniques and parallel processing have also decreased the run times of Monte Carlo codes and increases their usage. An advantage of using this technique is that very complex geometries can be modeled with high accuracy because it does not require a mesh structure. In addition, Monte Carlo methods have a continuous energy treatment for particle interaction cross-sections, as opposed to the discrete, multigroup approach commonly used in deterministic transport.

Because Monte Carlo codes are statistical in nature, their calculations do not give exact results. They give approximations of the results with associated statistical errors. To reduce these errors, the Monte Carlo technique requires a large number of particles to be simulated, which means it needs a large amount of time and computing power. As more particles enter a specific region, there are more data points in that region. With more points in the data set, variance in estimated quantities will decrease. In general, Monte Carlo codes accurately and efficiently calculate global, or system-wide, quantities, but do not work as well for detailed resolved parameters. However, Monte Carlo solutions are not global. The fluxes, reaction rates, and other characteristics of the radiation field are only obtained in volumes and on surfaces that are selected prior to a simulation. A major drawback of Monte Carlo methods is the extremely long run times required to achieve good statistical precision, particularly for problems with deep penetration, high degree of self-attenuation, or those of large physical scale.

\section{Deterministic Transport Methods}

Deterministic techniques for particle transport are used almost exclusively before the Monte Carlo technique became common and are still used frequently, although they are rarely used for radiation detection applications. Most detection simulations are done using Monte Carlo methods, in particular MCNP. However, deterministic codes which use finite elements to solve the transport equation are used in highly scattering scenarios like shielding calculations. Deterministic codes involve the discretization of the transport equation in each of its independent variables to generate a large system of linear equations. The solution of this linear system yields information about the particle population at every location in the discrete representation of the problem. This process can find solutions to problems that are previously unsolvable by hand as well as being fast and yielding very accurate results.

Many problems in radiation detection involve complicated scenarios with considerable scattering, and are extremely difficult and time consuming to run in MCNP. Deterministic calculations can provide significant speedups (10's to 100's of times faster) for some of these problems. In particular, problems with a low probability of any given photon reaching the detector -- heavily shielded or scattering problems - benefit from deterministic calculation, as do long dwell time problems. Deterministic problems solve the problem everywhere in space, at infinite time. However, pulse height tallies for detector response at present cannot be calculated deterministically.

The deterministic transport code used for the calculations in these test cases is called Attila, and is developed by Transpire Inc. Attila uses input to solve the first order form of the steady state 
linearized Boltzmann transport equation for each of its mesh cells. Attila solves this equation by discretizing it and solving iteratively for six variables in location, energy and direction. It utilizes finite difference, finite element, nodal, discrete ordinates (Sn) and Pn methods. As with the Monte Carlo technique, there are many other deterministic codes and methods, but Attila is used for the scenarios in this test case set [McGhee et al. 2007].

In general, deterministic methods tend to allow better resolution of local flux-related parameters, especially in low flux regions of the problem. Other advantages of deterministic methods are that they can be significantly faster than Monte Carlo methods for shielded and self-attenuating scenarios, they provide a solution everywhere, and the solution has no statistical noise. [Lewis et al. 1984] However, most deterministic codes rely on uniform Cartesian meshes, making them intractable for geometrically complex scenarios. In addition, discretization errors such as ray effects can be problematic in detection scenarios. While deterministic algorithms enable more comprehensive investigations of particle transport theory, they all generally possess an inherent shortcoming, (i.e. some discretization error). This error is a result of the limitation of computer storage, although it is large by past standards and continually increasing.

However, some kinds of problems are not well suited to deterministic simulation (at least, the discrete ordinates approach). Simple problems with little scatter may not benefit and timing or coincidence information is currently not included. Problems where the mesh must span a large range in length scales can be computationally expensive. Perhaps the biggest obstacle, though, to incorporating deterministic simulation directly into radiation detection calculations is found to be the level of user expertise required to run it. With a Monte Carlo simulation, barring errors in setup geometry, simulation error is primarily statistical and reflects precision. With deterministic simulation, convergence is determined by successively refining the discretization parameters (mesh size, number of angles in the scattering term, number of energy bins). Only by ensuring that the solution is stable upon further refinement is the user confident of an accurate answer; insufficiently converged runs can otherwise be difficult to identify, particularly for a non-expert.

\section{Coupled Transport Methods}

One combination of the two methods is referred to as the coupled method. In a previous NA-22 project, the Radiation Detection Scenario Analyst Toolbox (RADSAT) is developed using the coupled method. RADSAT is founded on a 3-dimensional deterministic radiation transport solver capable of efficiently computing the radiation field at all points in complex, large-scale problems (e.g. buildings). These results are then coupled to a Monte Carlo detector response simulator. The key challenge of this two-step coupled process is translating the angular flux information provided by a deterministic transport solution, to a definition of individual source particles for Monte Carlo transport. For a Monte Carlo source particle to be created, a particle's location, energy, and direction must be defined. All of this information can be derived from the discretized angular flux so long as the deterministic angular flux has sufficient fidelity in terms of angle, energy, and location for the detection scenario and spectrometer under investigation. [Burns et al. 2009, Casella et al. 2009, Shaver et al. 2009, Smith, et al. 2008]

To accomplish this, the geometry from the MCNP input deck is used to create a three dimensional solid model using Solid Works 3D (any 3D CADing program works). This simplified model is then converted into the Parasolid format, which is the required format for the Attila input. This Parasolid model is the basis for mesh structure that Attila defines as the system. Multigroup cross sections are then generated from material densities and compositions. Once 
all of the materials in the Solid Works model have associated cross section data, they are coupled together in Attila. Attila has a user-friendly graphical user interface (GUI) which is used to input the 3D model of the geometry. This model is then used to make a mesh of the system to discretize the geometry. Attila uses an arbitrary tetrahedral mesh structure. The multigroup cross section data is then assigned to each individual mesh component in the geometry. Mesh cells essentially are divided up sections, or volumes, of the geometry. The more cells that are specified, the smaller their volumes are, so the more detailed the results, but the longer the run time. Attila then solves the specified problem for each cell by iterating within the cells and coupling the problem among all of the cells.

\section{Hybrid Transport Methods}

Another approach for combining deterministic and Monte Carlo transport methods is the Consistent Adjoint Driven Importance Sampling (CADIS) method [Wagner et al. 1998]. The fundamental concept is to generate an approximate importance function from a fast-running deterministic adjoint calculation and use the importance map to construct variance reduction parameters that can accelerate tally convergence in the Monte Carlo simulation. The variance reduction parameters consist of space and energy-dependent weight window bounds and an importance-weighted (biased) source distribution. In the simulation, the weight windows are used to split or roulette particles that move toward relatively more or less important regions of phase space, respectively. The biased source ensures that particles are preferentially started where they are likely to contribute to the tally of interest. In addition, the source biasing parameters are consistent with the weight map; that is, source particles are sampled with weights that are at, or very near, the center of the window.

The CADIS method was developed to accelerate the estimation of an individual tally (e.g., a single cell tally with no energy bins). A newer hybrid method, the Forward-Weighted CADIS (FW-CADIS) method [Wagner et al. 2009], was developed to obtain relatively uniform statistical uncertainties across an arbitrary number of tallies (or tally bins). In the FW-CADIS method, a forward (as opposed to adjoint) deterministic calculation is initially performed and the results are used to construct an adjoint source that is weighted by the inverse of the forward flux in the regions of space and energy where the tallies are defined. Once the source has been computed, a deterministic adjoint calculation is performed and variance reduction parameters are computed using the CADIS methodology. The FW-CADIS method is flexible. It can be used to accelerate multiple, isolated tallies (e.g., cell or point-detector tallies) or it can be applied to large regions of space and/or energy.

The CADIS and FW-CADIS hybrid methods have been implemented in the ADVANTG code [Wagner et al. 2002, Mosher et al. 2009], which was developed to automate the process of generating variance reduction parameters for MCNP5. Given an MCNP input file and additional parameters for the deterministic calculation (e.g., spatial mesh, quadrature order, etc.), ADVANTG constructs an input for and drives the Denovo 3-D discrete ordinates package [Evans et al. 2010]. Denovo implements modern discretization schemes and solvers to generate solutions by the fastest and most robust methods available for structured grids. ADVANTG uses the output of Denovo to construct space and energy-dependent weight-window targets and a biased source, which are output as a WWINP file and SDEF cards, respectively. This output can be used directly with unmodified versions of MCNP5 and MCNPX. ADVANTG has been shown to produce highly-converged tally results in challenging detection problems [Mosher et al. 2009] and requires significantly less effort from the user than MCNP's stochastic weight window generator [Smith et al. 2005]. 


\section{Performance Metrics}

In this new landscape of method and algorithm development, confirmation of a code's performance is essential. In practice, the utility of a code clearly depends on its accuracy and the computational resources required, but code performance is a not a simple, single, or sometimes even quantitative metric. It is a series of metrics that are interrelated and problem specific. The two general categories of performance of a code are quality, sometimes referred to as "solution accuracy" (how good of an answer does it give?) and time to solution (how long does it take to get that answer?). However, solution accuracy is not necessarily fixed across a problem, and may vary spatially or with energy. Also, many times solution accuracy and time to solution are inversely proportional, meaning it may take longer to get a better answer.

A suite of test cases provides a basis for testing radiation transport codes on relevant problems and help identify gaps in existing techniques which can in turn drive algorithm and method development. Comparing various test cases to experimental data and/or existing codes can help to define performance of the code, but it is important to have clear expectations on a problem-by-problem basis of what, exactly, will be compared. This is done by knowledge of detection metrics used on specific problems. Of course, there is no guarantee that this approach will confirm the performance of a particular computational algorithm for all cases, but it is currently the best approach. Even with the comparison data given here, code developers and users must decide which metrics will be most beneficial for specific problems. A discussion of various performance metrics and factors influencing them as well as a diagram of the metrics factors follows.

\section{Code Quality (Solution Accuracy)}

Confirmation of a code's quality, or solution accuracy, depends on a variety of factors that traditionally are placed into two categories: verification, which ensures that the code works as intended, and validation, which ensures that the results match experimental data. However, estimating actual error in a code's output can only been done for a few methods, and usually these estimates are grossly approximate and possible even misleading. The best methods of validation and verification are comparison of other algorithms designed for similar applications and comparison to standards in a set of test cases, which are tied to experimental data, if possible.

\section{Verification}

The simulation software must go through a verification process to demonstrate that the equations are being solved correctly and that there are no "bugs" in the code, to insure that it does what is intended. One important method of verification is the ability to represent the problem sufficiently accurately within the code, and to encompass the relevant physics (appropriate cross sections, etc.). In some cases, a 1-D simulation may provide a sufficiently good representation of a problem, while many other cases require full 3-D representations. Therefore, verification includes internal/system design verification, functional requirement verification, and coding verification. This involves multiple reviews and beta testing of the code. This is sometime referred to as end users trying to "break" the code, or "make it crash", to find bugs that the developers may not know about. Running ADVANTG for the test cases by multiple users (both at ORNL and PNNL) is one of the last steps in this process. These verification steps are usually accomplished by the developers, or reviewers. 
The user supplied inputs also should go through a verification process, although usually a less formal one. Accuracy in the output depends on having an accurate model of the scenario. This idea is usually colloquially expressed through a corollary statement of "garbage in, garbage out". This encompasses having appropriate geometry, material, and source representations, approximations, convergence criteria or other refinement of the solution, making sure the appropriate output is requested, making sure variance reduction techniques don't change the results, and other such issues. For a Monte Carlo approach, this may include the number of particles thrown. For a deterministic approach, this may include the level of discretization of the variables that is applied. Separately, the user must also verify that the problem they are trying to solve with the code is in the specified range over which the code has been validated. In other words, to ensure solution accuracy, the code must work for the type of problem being solved. These steps are usually accomplished by the users.

\section{Validation}

Once the code and inputs are verified, models in the software can be validated over some specified range of conditions. This can be aided by a predefined set of test cases that are well understood to ensure that the results match experimental data. However, there are different metrics of what in the experimental data needs to match. For example, in a gamma spectroscopy problem, integrated counts under a few key energy peaks in the detector (perhaps after a background subtraction) are a common metrics. For other problems, total counts at the detector integrated over all energies may be the most relevant metric (although a minimum energy may still need to be defined). Other times the user might be interested in a whole spectrum or template matching. Also, the level of accuracy desired may change. For example, it may be more important to get an answer fast, than exactly correct. The problem specific question that must be asked is, how good is good enough?

For hybrid or Monte Carlo methods, accuracies based on counting statistics can be defined for specific metrics (per energy bin, or per all energies, etc.); MCNP's figure of merit goes a step further in incorporating the tradeoff (for Poisson statistics) between computational time and accuracy and is a particularly useful gauge of hybrid methods. However, deterministic simulations do not offer a similar metric. Solution accuracy or matching experimental data alone is not the only basis for comparison, since most of the 3-D transport codes should converge to close the same answer given enough time/computational detail.

\section{Time to Solution}

Confirmation of a code's time to solution is more than just the code's running time, or speed. In fact, the total time to a solution involves the time it takes to set up the input for the run (preprocessing), the time it takes the code to run (computer runtime), and the time it takes to analyze the results (post-processing).

\section{Pre-Processing}

The first step in running the code is the preprocessing of setting up the inputs. Factors that can influence this include how user friendly (or how easy the code is to use), whether it has a GUI, or input decks, how complex the problem is, and what level of experience, or expertise the user has. For example, MCNP takes some expertise, but proper use of variance reduction techniques, such as weight windowing takes more expertise yet. Finalizing the input includes 
building the geometry and putting in sources, as well as adjusting (attempting to optimize) the run parameters in order to improve speed or accuracy and debugging the input. For a new problem, this can often include multiple iterations in order to debug or to establish appropriate parameters. For a familiar problem, setup time can be significantly reduced, since it's just a matter of preparing the case (or building off an old one) rather than iterating to "figuring out" how to run it. For some cases the time it takes to properly set up variance reduction or optimize parameters can be greater than the savings in runtime.

\section{Computer Runtime}

Computer runtime is influenced by how fast the code itself runs, but also what resources are available on which to run it. Some codes can be parallelized on multiple processors, while others cannot. It is also influenced by the experience and expertise of the user, how complicated a problem is, and how the user sets up the problem can effect both the overhead to read the input into the code and possibly the time it takes to calculate the particle transport. The operator must generally choose the fidelity to which the code is run, understanding that usually a higher level of fidelity and accuracy result in a longer run time. The physics mechanisms that dominate the problem will also affect the runtime. How optimized the parameters are and how much variance reduction is used are a couple of examples of how runtime can be affected by the actions of the users.

The nature of these choices differ depending on the class of code being used: for Monte Carlo methods, an accurate solution is gradually accumulated through the simulation of increasing numbers of particles, while for a deterministic solution, an accurate solution is approached via increasingly fine discretization of parameters (generally space, energy, and angle). It is important to note that accuracy is not constant everywhere in a problem - some energies or locations may be more accurate than others - so even specifying a particular accuracy goal must be done with some care, and may involve problem specific choices as to which metrics are most relevant. For some codes, more careful problem setup can result in both reduced runtimes and higher accuracy.

At its simplest, computer runtime may be assessed as the total time solution normalized by processing capacity - akin to time per processor $\mathrm{MHz}$. This is what is done for the cases in this document. This of course does not take into account factors such as memory usage, bus speeds and disk speeds, or overhead associated with reading input decks and beginning a calculation (which can be significant, especially where a problem is relatively simple to calculate or where a method is relatively complex). Also, a coupled or hybrid code will have runtimes associated with both deterministic and Monte Carlo portions of the calculation which will need to be accounted for. The runtimes given in each test case below are normalized within the case to processor speed for a comparison, but are not normalized between cases, to stay consistent with the references for each case. Computational times, unless otherwise noted, are given for runs on a machine with 4 dual-core processors running at $2.66 \mathrm{GHz}$ (Intel Xeon 5150) and 32 GB memory. More information on the computers used in each test case can be found in the references they were taken from.

\section{Post-Processing}

Analyzing the output of the code through data analysis, normalization, plotting, and other techniques are often overlooked in discussions of time to a solution, but they are important. This can be affected by user experience and expertise, but it can also be influenced by the output options and formats of the code. 


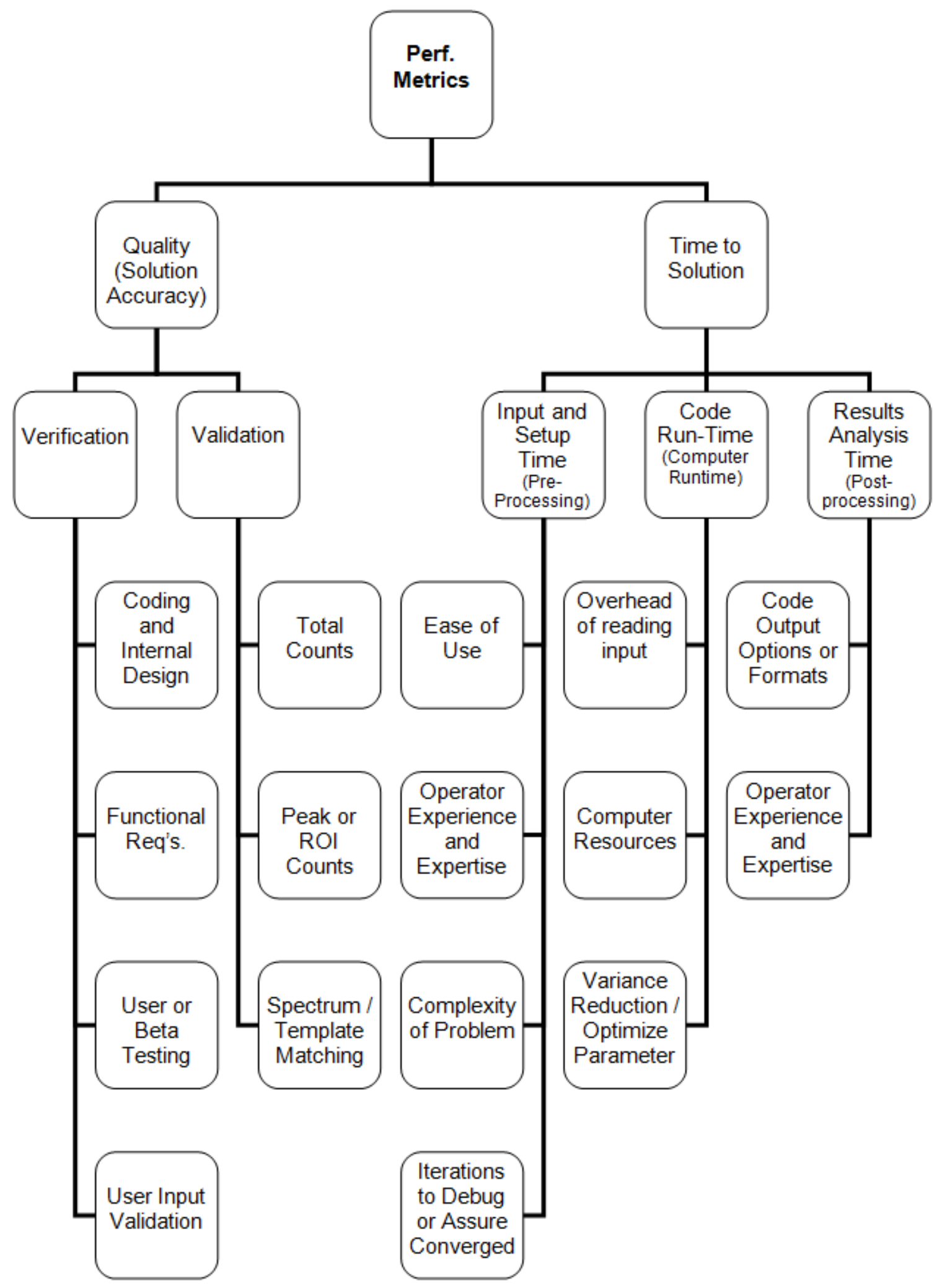




\section{Problem Setup}

The discussion above illustrates why using test cases is a well-established and recognized part of particle transport algorithm and method development. For these reasons, benchmarking activity has greatly increased in importance to the nuclear community recently. Extensive computational benchmarking sets have been developed and heavily used for criticality and for analytical methods of reactor analysis [Ganapol 2008]. However, no benchmarking sets have been made for radiation detection scenarios. In addition to providing a common standard for algorithm and method development, a computational radiation detection scenario benchmark set is a point of reference for detector developers and detector scenario analysts. While this approach provides a general framework for describing, understanding, and re-running the test cases, this does not yet include detailed cross-code comparisons or evaluations. Future work will broaden the cases to include other classes of transport calculations (low-dimension models, deterministic transport, and hybrid methods), and will include more extensive cross-code comparisons in order to better understand the tradeoffs in computation time, accuracy, and operator time. Therefore, the test cases described here will form the basis for a benchmark set in the future which will include the results from the hybrid method along with the Monte Carlo and Coupled methods described below.

This report presents 9 test problems to guide testing and development of hybrid calculations for the ADVANTG code at ORNL. These test cases can be used for comparing different types of radiation transport calculations, as well as for guiding the development of variance reduction methods. Cases are drawn primarily from existing or previous calculations with a preference for cases which include experimental data, or otherwise have results with a high level of confidence, are non-sensitive, and represent problem sets of interest to NA-22. From a technical perspective, cases are chosen to span a range of difficulty and to include gamma transport, neutron transport, or both and represent different important physical processes.

Following test case identification, existing information about geometry, measurements, and previous calculations were assembled. Monte Carlo results (MCNP decks) were reviewed or created and re-run in order to attain accurate computational times and to verify agreement with experimental data, when present. Test case information was then conveyed to ORNL in order to guide testing and development of hybrid calculations. The results of those ADVANTG calculations were then sent to PNNL for compilation. This is a report describing the details of the selected test cases and results from available transport codes. The later sections of this report provide a short description of the test cases and specifics such as source definition, geometry and materials.

The 9 Test Problems are:

1) PANDA Neutron Cases (n)

2) Neutron Transport (n)

3) Mulch Box ( $\mathrm{y}$ )

4) Skyshine Gamma Background ( $\mathrm{Y}$ )

5) Spent Fuel Cask Gamma-Scanner ( $\mathrm{Y}$ )

6) Radiation Portal Monitor ( $(y)$

7) Radiography ( $\mathrm{Y}$ )

8) Polyethylene Coupled Neutron Gamma $(n, y)$

9) $\mathrm{UF}_{6}$ Cylinder $(n, y)$ 


\subsection{PANDA Neutron Cases}

\subsection{Description}

These cases represent relatively simple neutron transport problems, with bare sources and moderated detectors. The cases are drawn from Chapter 14 of the Passive Nondestructive Assay of Nuclear Materials book, commonly referred to as PANDA, which describes some radiation detection related scenarios and is probably the closest there is to an existing neutron test case in the open literature. These cases are very good test cases because they are well defined, simple to model, and involve several different neutron spectra from the varying moderation of californium-252 sources [Stewart, 1991].

Chapter 14.4.1 describes a series of one inch detectors in a polyethylene moderator ring with a source in the middle. The number of detectors is changed in each case. The geometry is shown below in Figure 1.1. Chapter 14.4.2 describes optimizing the moderator thickness for thermal-neutron sensitive helium-3 tube detectors. This geometry is shown below in Figure 1.1, where the amount of polyethylene on both the front and back of the detector are varied between two and eight centimeters.

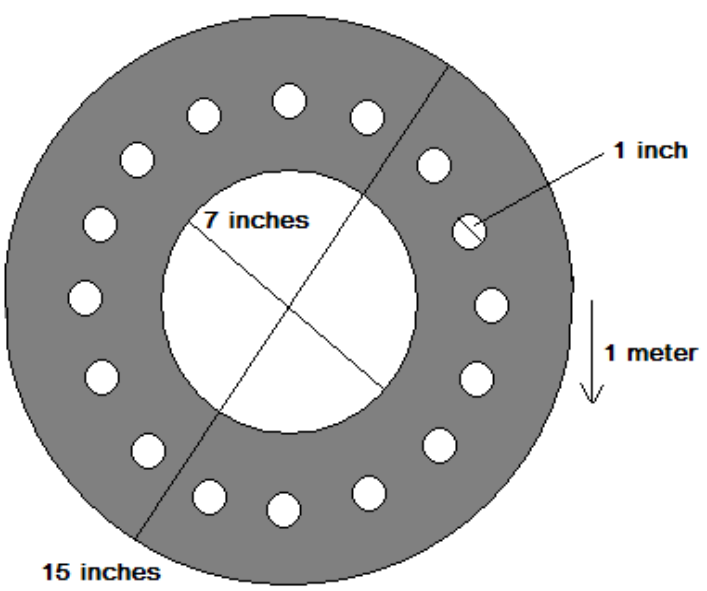

Figure 1. The geometry for the 14.4.1 PANDA case. [Stewart 1991].

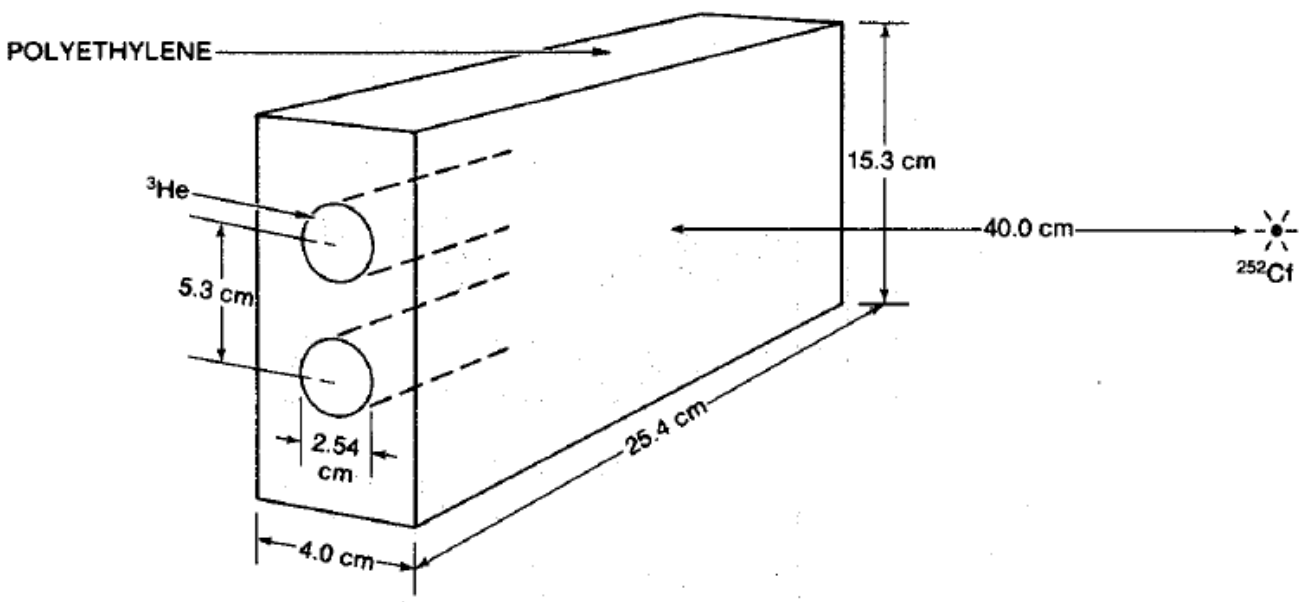

Figure 22. The geometry for the 14.4.2 PANDA case. [Stewart 1991]. 


\subsection{Comparison of Results}

For the PANDA Chapter 14.4.1 case each MCNP case was run with $10^{6}$ particles. To spatially discretize the geometry in Attila by using tetrahedral meshes, the cylinders were converted to hexagons. This change in the geometry can be seen below in Figure 1.3. The global mesh size was $40 \mathrm{~cm}$, the mesh in the moderator is $10 \mathrm{~cm}$ and the mesh size in the detectors is $2 \mathrm{~cm}$. The case was run with S8P2 Triangle Chebychev Labotto and Galerkin Scattering and upscatter acceleration turned on. The SCALE44 group neutron cross sections are used.

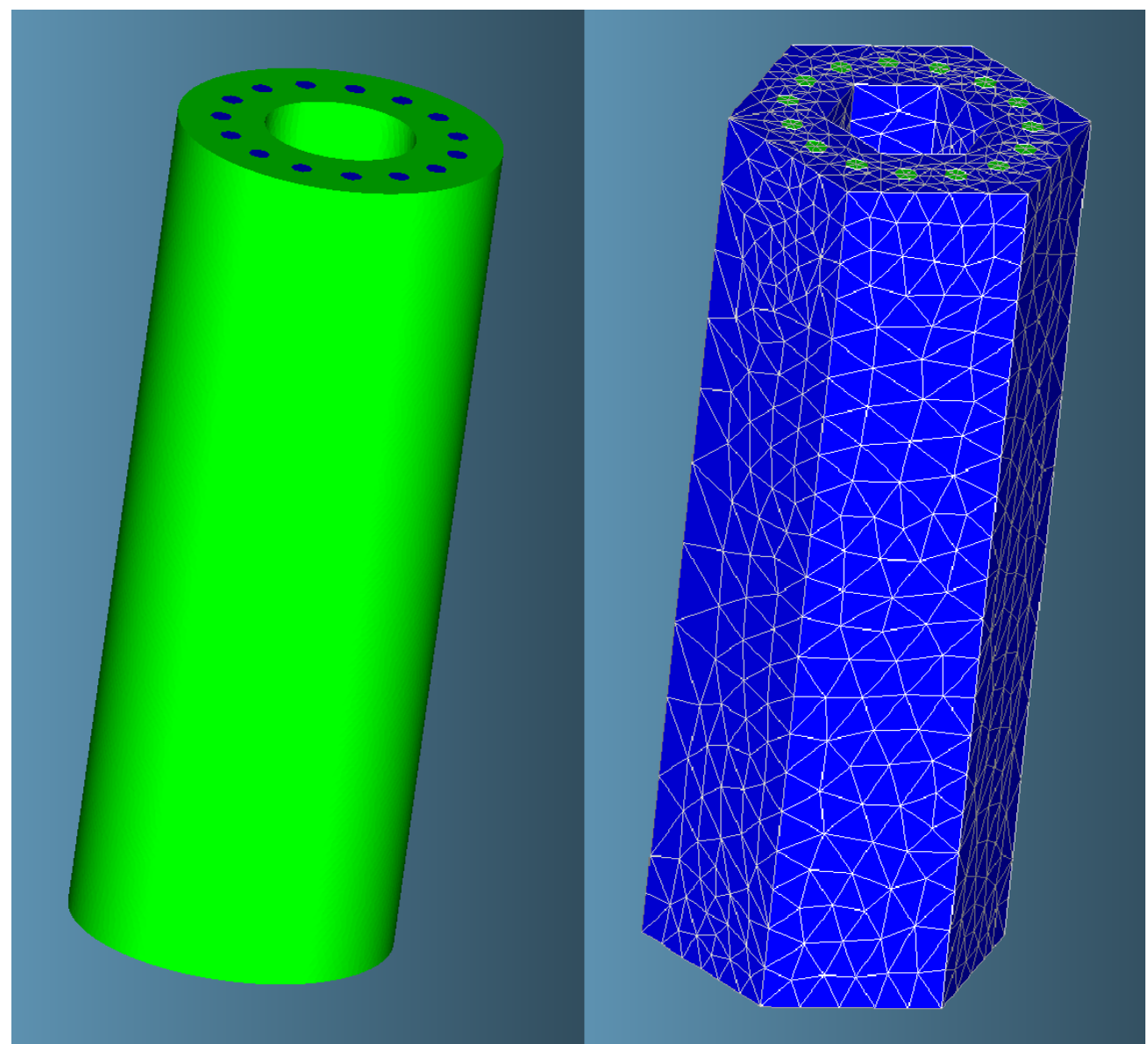

Figure 3. The Attila geometry approximations for the 14.4.1 PANDA case. [Stewart 1991].

The results as presented in PANDA 14.4.2 along with the comparisons from MCNP and RADSAT are shown below in Figure 1.4. The average difference of the MCNP cases is $+3 \%$, while the average difference of the RADSAT cases was $-5 \%$. The MCNP cases took on average 3.5 minutes. All of the MCNP cases took approximately the same amount of time to run. The RADSAT cases were stopped after 10 outer iterations for each case, which took between 5 and 11 hours to complete. In Attila, the more detectors modeled, the more meshes needed, so the longer the runtimes. This clearly shows that to converge on the same answer MCNP takes orders of magnitude less time to complete the cases. 


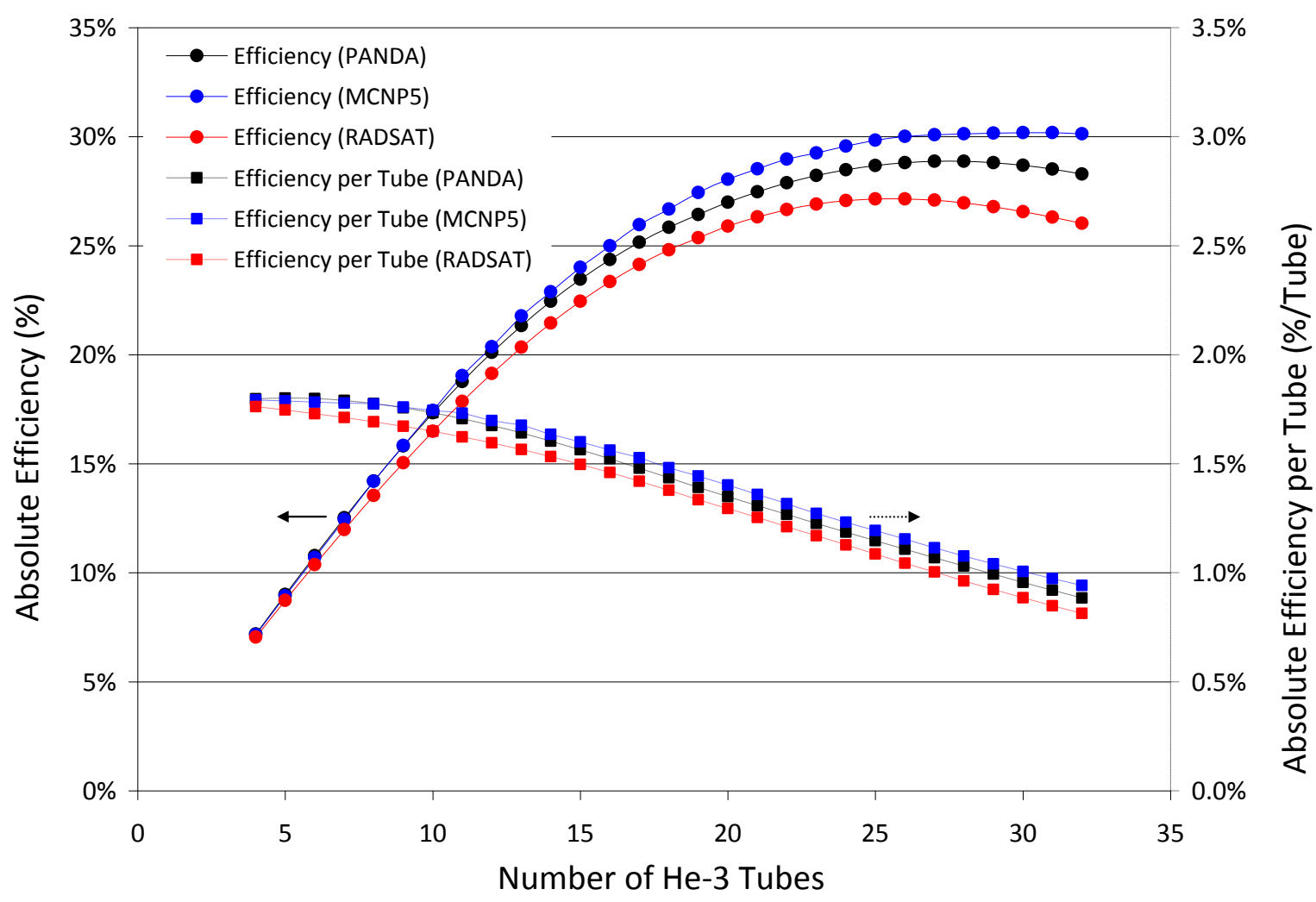

Figure 4. Results for the 14.4.1 PANDA case. [Stewart 1991].

The results as presented in PANDA 14.4.2 are shown below in Figure 1.5. Note that the lack of smoothness in the shape of the contour plot indicates some amount of uncertainty in the results, however, the uncertainties are not given in PANDA and their source is unkown. Comparison to the PANDA results are given in Tables 1.1 and 1.2 below as relative deviation from the PANDA values for all moderator thicknesses. Cases where the absolute relative deviation is less than $5 \%$ are color coded green indicating good agreement, cases whose deviation are between $5 \%$ and $10 \%$ are yellow indicating marginal agreement, and cases whose deviation are greater than $10 \%$ are red, indicating poor of an agreement. Note that all runtimes have been adjusted by the processor speed to fairly compare runtimes between codes run on different computers.

Each MCNP case is run with $10^{8}$ particles. The results from the MCNP cases are shown below in Figure 1.6 and Table 1.1. The deviation from the PANDA cases are all less than $10 \%$, with the highest being $9 \%$ and the average deviation being only $2 \%$ per case. 


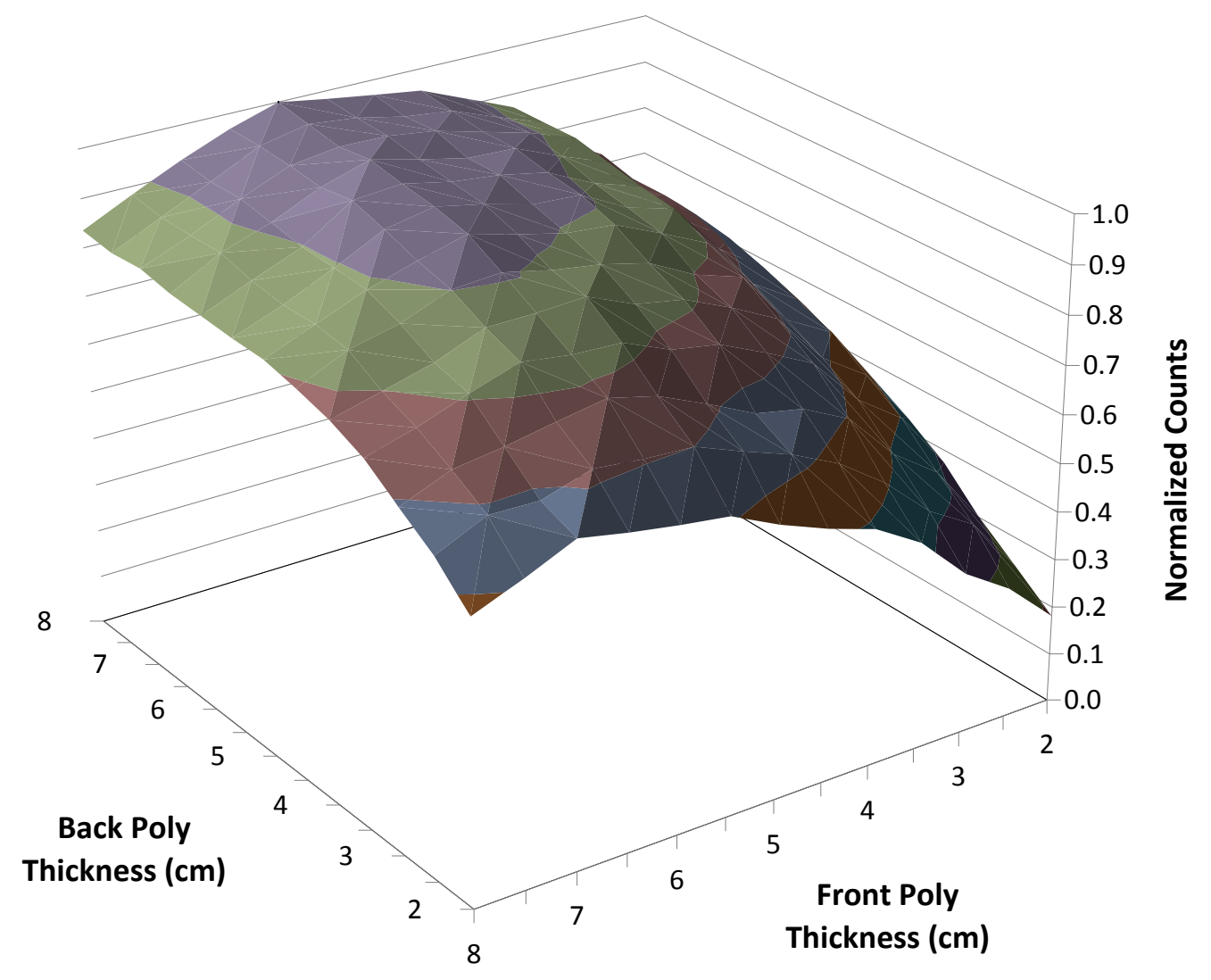

Figure 5. Normalized Results from PANDA 14.4.2.

The cross section set that is used for the RADSAT case in Attila is the SCALE 44 set, which is made for light water reactors. Therefore, there are many thermal groups, making those neutrons well represented, while fast neutrons are not. These cases are run with S8P1 Triangle Chebychev Labotto quadrature and Galerkin scattering. The Attila case had a global mesh size of 1 , a mesh size of 0.1 in the helium tubes, and $0.01 \mathrm{~cm}$ in the polyethylene shield. The results from the RADSAT cases are shown below in Figure 1.4 and Table 1.2. The RADSAT deviation from the PANDA cases are average of $4 \%$ with a maximum of $17 \%$. However, for the half of the cases with the most moderator, the average deviation is $1 \%$ and the maximum is $4 \%$. The average for the half of the cases with the least amount of moderator is $7 \%$, with a maximum of $17 \%$. This illustrates the RADSAT is doing a very good job with the thermal neutrons, but not with the fast neutrons. This is due to the cross section set that is used as described earlier.

The shortest runtime in MCNP is the $2 \mathrm{~cm} \times 2 \mathrm{~cm}$ moderator at 169 minutes and the longest runtime is the $8 \mathrm{~cm} \times 8 \mathrm{~cm}$ moderator at 217 minutes. The average runtime for the cases is 192 minutes. The runtime in RADSAT is 38 minutes per run. However, to increase the number of energy groups to get the same statistical agreement as MCNP, it is estimated that the run times would be 3.6 times longer, making them around 70 minutes per run, or $\sim 1.5$ times faster than MCNP. This would also include much more time up front generating a problem specific cross section set, rather than using the existing SCALE44 set, greatly increasing the total time to solution. Also, with minimal variance reduction, runtimes in MCNP could be decreases to faster than the RADSAT runs. The ADVANTG runs yielded essentially identical results to MCNP and were on average a factor of twelve slower than MCNP. Therefore, RADSAT has the fastest run time, but the total time to solution is the fastest in MCNP when taking into account having to make a special cross section library for the RADSAT runs. 


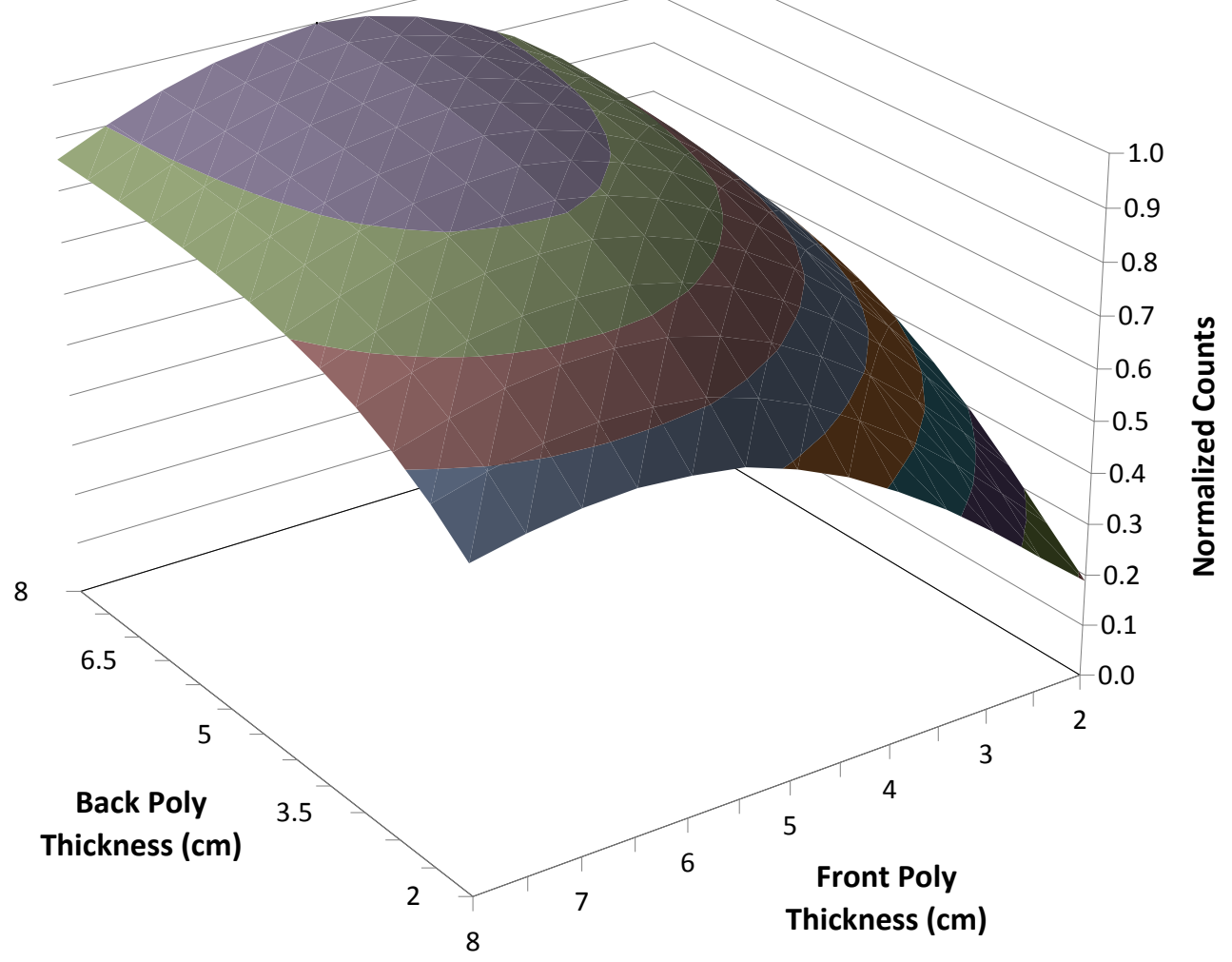

Figure 6. Normalized results from MCNP 14.4.2.

Table 1.1. MCNP results compared to PANDA 14.4.2.

\begin{tabular}{|c|c|c|c|c|c|c|c|c|c|c|c|c|c|c|}
\hline \multicolumn{2}{|c|}{$\begin{array}{l}\text { Rel. } \\
\text { Diff. }\end{array}$} & \multicolumn{13}{|c|}{ FRONT POLY THICKNESS (cm) } \\
\hline & & 2 & 2.5 & 3 & 3.5 & 4 & 4.5 & 5 & 5.5 & 6 & 6.5 & 7 & 7.5 & 8 \\
\hline \multirow{13}{*}{ 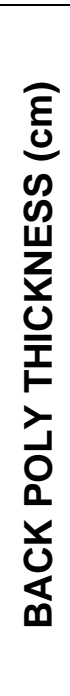 } & 2 & $4 \%$ & $2 \%$ & $4 \%$ & $2 \%$ & $0 \%$ & $5 \%$ & $5 \%$ & $3 \%$ & $3 \%$ & $1 \%$ & $2 \%$ & $2 \%$ & $6 \%$ \\
\hline & 2.5 & $7 \%$ & $4 \%$ & $4 \%$ & $1 \%$ & $0 \%$ & $2 \%$ & $3 \%$ & $1 \%$ & $2 \%$ & $1 \%$ & $4 \%$ & $1 \%$ & $4 \%$ \\
\hline & 3 & $9 \%$ & $1 \%$ & $4 \%$ & $1 \%$ & $2 \%$ & $7 \%$ & $5 \%$ & $3 \%$ & $0 \%$ & $2 \%$ & $3 \%$ & $2 \%$ & $3 \%$ \\
\hline & 3.5 & $5 \%$ & $1 \%$ & $3 \%$ & $1 \%$ & $1 \%$ & $1 \%$ & $4 \%$ & $1 \%$ & $1 \%$ & $1 \%$ & $1 \%$ & $2 \%$ & $2 \%$ \\
\hline & 4 & $6 \%$ & $0 \%$ & $4 \%$ & $1 \%$ & $1 \%$ & $1 \%$ & $3 \%$ & $1 \%$ & $1 \%$ & $0 \%$ & $3 \%$ & $2 \%$ & $1 \%$ \\
\hline & 4.5 & $6 \%$ & $0 \%$ & $5 \%$ & $1 \%$ & $2 \%$ & $2 \%$ & $3 \%$ & $3 \%$ & $0 \%$ & $0 \%$ & $2 \%$ & $0 \%$ & $1 \%$ \\
\hline & 5 & $5 \%$ & $1 \%$ & $5 \%$ & $0 \%$ & $1 \%$ & $1 \%$ & $3 \%$ & $2 \%$ & $2 \%$ & $0 \%$ & $1 \%$ & $1 \%$ & $1 \%$ \\
\hline & 5.5 & $3 \%$ & $1 \%$ & $4 \%$ & $1 \%$ & $2 \%$ & $1 \%$ & $3 \%$ & $2 \%$ & $1 \%$ & $1 \%$ & $2 \%$ & $3 \%$ & $3 \%$ \\
\hline & 6 & $4 \%$ & $0 \%$ & $4 \%$ & $0 \%$ & $2 \%$ & $1 \%$ & $2 \%$ & $2 \%$ & $1 \%$ & $2 \%$ & $2 \%$ & $3 \%$ & $3 \%$ \\
\hline & 6.5 & $5 \%$ & $1 \%$ & $4 \%$ & $0 \%$ & $1 \%$ & $1 \%$ & $3 \%$ & $2 \%$ & $1 \%$ & $0 \%$ & $2 \%$ & $2 \%$ & $3 \%$ \\
\hline & 7 & $3 \%$ & $2 \%$ & $6 \%$ & $1 \%$ & $1 \%$ & $2 \%$ & $2 \%$ & $2 \%$ & $0 \%$ & $0 \%$ & $1 \%$ & $3 \%$ & $3 \%$ \\
\hline & 7.5 & $5 \%$ & $3 \%$ & $5 \%$ & $0 \%$ & $2 \%$ & $2 \%$ & $3 \%$ & $2 \%$ & $0 \%$ & $0 \%$ & $2 \%$ & $3 \%$ & $4 \%$ \\
\hline & 8 & $4 \%$ & $1 \%$ & $5 \%$ & $1 \%$ & $2 \%$ & $1 \%$ & $2 \%$ & $2 \%$ & $1 \%$ & $0 \%$ & $1 \%$ & $2 \%$ & $3 \%$ \\
\hline
\end{tabular}




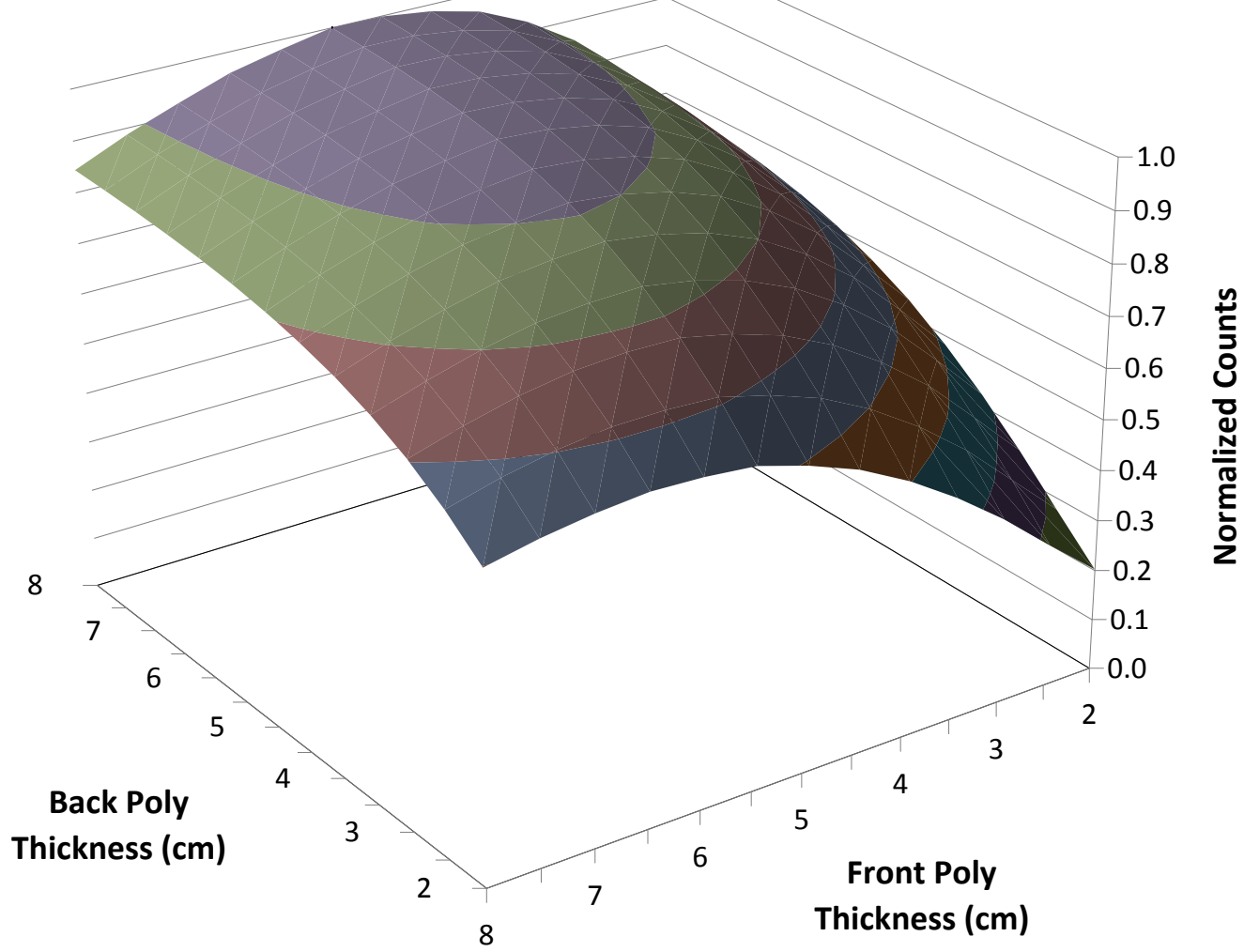

Figure 7. Normalized results from RADSAT 14.4.2.

Table 1.2. RADSAT results compared to PANDA 14.4.2.

\begin{tabular}{|c|c|c|c|c|c|c|c|c|c|c|c|c|c|c|}
\hline \multicolumn{2}{|c|}{$\begin{array}{l}\text { Rel. } \\
\text { Diff. }\end{array}$} & \multicolumn{13}{|c|}{ FRONT POLY THICKNESS (cm) } \\
\hline & & 2 & 2.5 & 3 & 3.5 & 4 & 4.5 & 5 & 5.5 & 6 & 6.5 & 7 & 7.5 & 8 \\
\hline \multirow{13}{*}{ 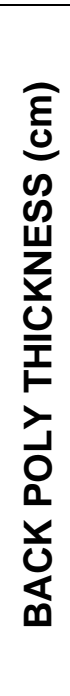 } & 2 & $12 \%$ & $4 \%$ & $10 \%$ & $2 \%$ & $3 \%$ & $7 \%$ & $6 \%$ & $3 \%$ & $2 \%$ & $0 \%$ & $4 \%$ & $0 \%$ & $4 \%$ \\
\hline & 2.5 & $15 \%$ & $2 \%$ & $9 \%$ & $5 \%$ & $3 \%$ & $4 \%$ & $3 \%$ & $1 \%$ & $1 \%$ & $0 \%$ & $2 \%$ & $1 \%$ & $2 \%$ \\
\hline & 3 & $17 \%$ & $5 \%$ & $10 \%$ & $3 \%$ & $5 \%$ & $9 \%$ & $6 \%$ & $3 \%$ & $0 \%$ & $1 \%$ & $1 \%$ & $0 \%$ & $2 \%$ \\
\hline & 3.5 & $13 \%$ & $5 \%$ & $8 \%$ & $3 \%$ & $4 \%$ & $3 \%$ & $5 \%$ & $1 \%$ & $1 \%$ & $2 \%$ & $1 \%$ & $0 \%$ & $0 \%$ \\
\hline & 4 & $14 \%$ & $6 \%$ & $10 \%$ & $4 \%$ & $4 \%$ & $3 \%$ & $4 \%$ & $1 \%$ & $0 \%$ & $1 \%$ & $1 \%$ & $1 \%$ & $1 \%$ \\
\hline & 4.5 & $14 \%$ & $6 \%$ & $11 \%$ & $3 \%$ & $6 \%$ & $4 \%$ & $3 \%$ & $3 \%$ & $1 \%$ & $2 \%$ & $0 \%$ & $1 \%$ & $0 \%$ \\
\hline & 5 & $12 \%$ & $7 \%$ & $10 \%$ & $4 \%$ & $4 \%$ & $3 \%$ & $4 \%$ & $2 \%$ & $2 \%$ & $1 \%$ & $1 \%$ & $0 \%$ & $0 \%$ \\
\hline & 5.5 & $11 \%$ & $7 \%$ & $9 \%$ & $3 \%$ & $5 \%$ & $3 \%$ & $4 \%$ & $2 \%$ & $2 \%$ & $2 \%$ & $0 \%$ & $1 \%$ & $1 \%$ \\
\hline & 6 & $12 \%$ & $6 \%$ & $9 \%$ & $4 \%$ & $6 \%$ & $3 \%$ & $3 \%$ & $2 \%$ & $2 \%$ & $3 \%$ & $1 \%$ & $1 \%$ & $1 \%$ \\
\hline & 6.5 & $12 \%$ & $7 \%$ & $9 \%$ & $4 \%$ & $5 \%$ & $3 \%$ & $4 \%$ & $2 \%$ & $2 \%$ & $1 \%$ & $0 \%$ & $0 \%$ & $2 \%$ \\
\hline & 7 & $10 \%$ & $8 \%$ & $12 \%$ & $3 \%$ & $4 \%$ & $4 \%$ & $3 \%$ & $2 \%$ & $1 \%$ & $1 \%$ & $0 \%$ & $1 \%$ & $1 \%$ \\
\hline & 7.5 & $13 \%$ & $9 \%$ & $10 \%$ & $5 \%$ & $5 \%$ & $4 \%$ & $4 \%$ & $2 \%$ & $1 \%$ & $1 \%$ & $0 \%$ & $1 \%$ & $2 \%$ \\
\hline & 8 & $12 \%$ & $7 \%$ & $11 \%$ & $5 \%$ & $5 \%$ & $3 \%$ & $3 \%$ & $2 \%$ & $2 \%$ & $1 \%$ & $0 \%$ & $1 \%$ & $1 \%$ \\
\hline
\end{tabular}




\subsection{Neutron Transport Case}

\subsection{Description}

This test case problem measures the response of a shielded He-3 neutron detector to a Cf-252 source, and provides a relatively simple neutron transport case with a range of shielding options. The source can either be a point source or a volume source and the detector can be separated by shields of various thicknesses as displayed in Figure 2.1, where three shields are $20 \mathrm{~cm}, 10 \mathrm{~cm}$, and $1 \mathrm{~cm}$. The three shields are typically polyethylene or air, but can be any material. While this case has no experimental results, it is used as neutron test case for verification and validation of the RADSAT software during its development and as a test case in the RADSAT tutorial.

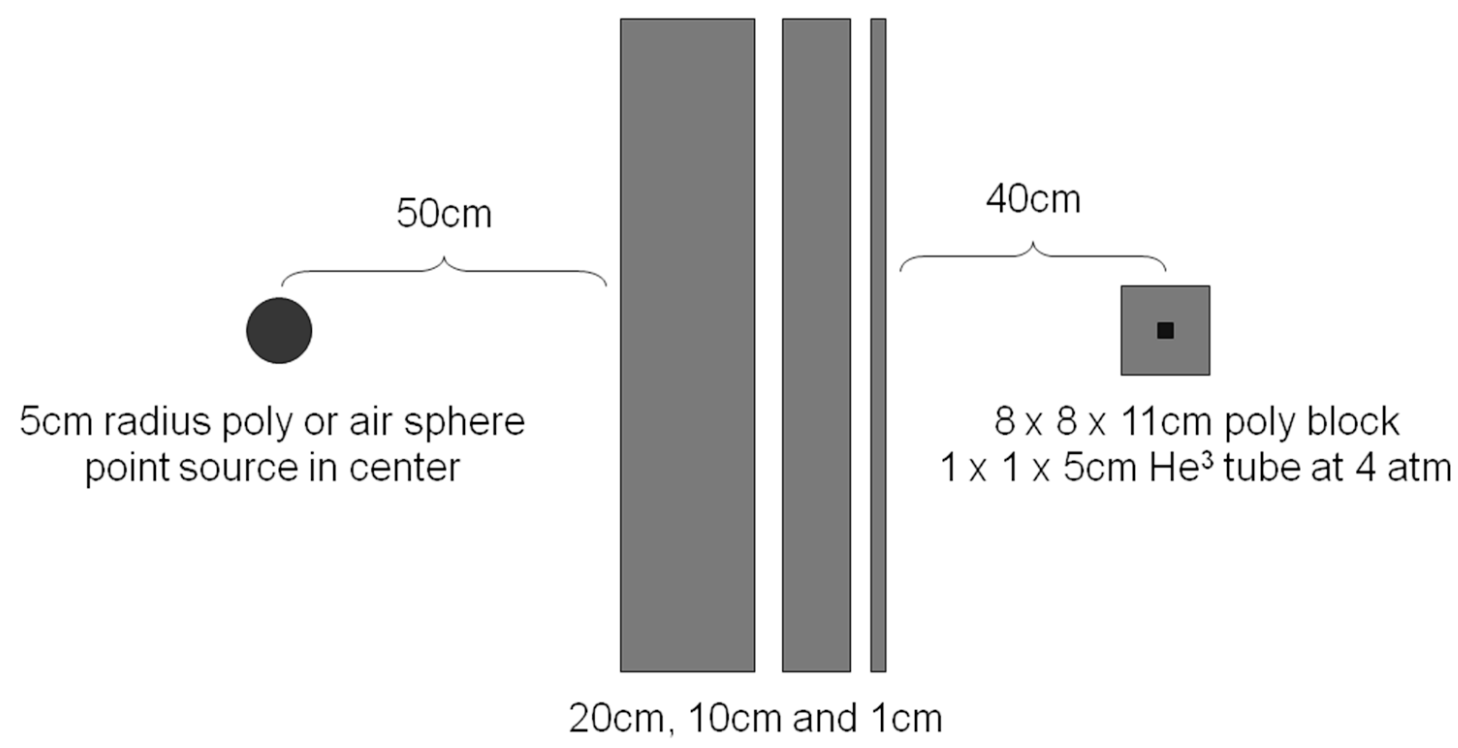

Figure 8. The geometry for the Neutron Transport case.

\subsection{Comparison of Results}

For the RADSAT case, the Triangular Chebyshev Legendre quadrature set with S10P2 is used. Attila mesh sizes used are: global $=1 \mathrm{~cm}$, polyethylene $=0.1 \mathrm{~cm}$, and ${ }^{3} \mathrm{He}=0.01 \mathrm{~cm}$. Runtimes for MCNP reflect the tracking of $10^{9}$ source neutrons. For the ADVANTG run, there were some issues with ray effects in the streaming problem of the adjoint calculation as can be seen below in Figure 2.2. 


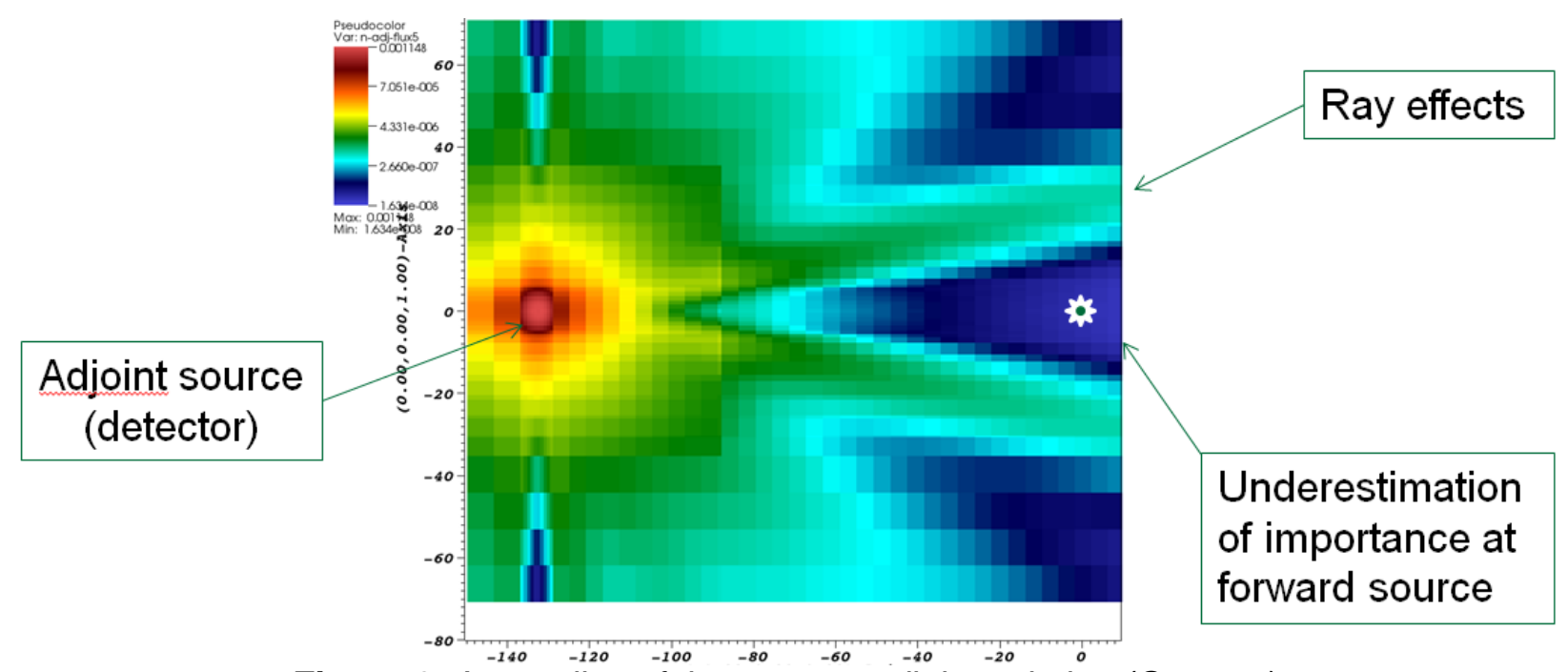

Figure 9 A $45^{\circ}$ slice of the neutron adjoint solution (Group 5)

The results are below in Table 2.1. As can be seen, they results are very similar and sometimes MCNP is faster, while other time ADVANTG is faster.

Table 2.1. Results Summary

\begin{tabular}{|c|c|c|c|c|}
\hline Test & $\begin{array}{c}\text { No } \\
\text { Shield }\end{array}$ & $\begin{array}{c}\text { Right (thin) } \\
\text { Shield }\end{array}$ & $\begin{array}{c}\text { Center (medium) } \\
\text { Shield }\end{array}$ & $\begin{array}{c}\text { Left (thick) } \\
\text { Shield }\end{array}$ \\
\hline $\begin{array}{c}\text { MCNP } \\
\text { Counts/cm } \\
\text { run-time }\end{array}$ & $\begin{array}{c}7.95 \times 10^{-7} \\
73 \mathrm{~min}\end{array}$ & $\begin{array}{c}6.78 \times 10^{-7} \\
73 \mathrm{~min}\end{array}$ & $\begin{array}{c}1.88 \times 10^{-7} \\
151 \mathrm{~min}\end{array}$ & $\begin{array}{c}2.68 \times 10^{-8} \\
305 \mathrm{~min}\end{array}$ \\
\hline $\begin{array}{c}\text { ADVANTG } \\
\text { Counts/cm } \\
\text { run-time }\end{array}$ & - & $\begin{array}{c}6.72 \times 10^{-7} \\
270 \mathrm{~min}\end{array}$ & $\begin{array}{c}1.91 \times 10^{-7} \\
5 \mathrm{~min}\end{array}$ & $\begin{array}{c}2.69 \times 10^{-8} \\
1.5 \mathrm{~min}\end{array}$ \\
\hline $\begin{array}{c}\text { Relative } \\
\text { Difference (\%) }\end{array}$ & - & $-0.86 \%$ & $1.5 \%$ & $0.48 \%$ \\
\hline $\begin{array}{c}\text { RADSAT } \\
\text { Counts/cm }\end{array}$ & $8.01 \times 10^{-7}$ & $6.99 \times 10^{-7}$ & $1.91 \times 10^{-7}$ & $2.51 \times 10^{-8}$ \\
run-time & $96 \mathrm{~min}$ & $105 \mathrm{~min}$ & $197 \mathrm{~min}$ & $293 \mathrm{~min}$ \\
\hline $\begin{array}{c}\text { Relative } \\
\text { Difference (\%) }\end{array}$ & $0.75 \%$ & $3.1 \%$ & $1.6 \%$ & $-6.3 \%$ \\
& $32 \%$ & $43 \%$ & $30 \%$ & $-3.9 \%$ \\
\hline
\end{tabular}

The plot below in Figure 2.3 shows that for little to no shielding, using analog MCNP is the fastest because it is such a simple problem, it is not worth the computational overhead of including the deterministic codes. This is especially true if the setup time is also taken into account. For very thin shields deterministic codes have to make very fine meshes, which increases the total number of meshes, and therefore, the runtime. The overhead of the adjoint solution only becomes effective in reducing run time with a thick enough shield. 


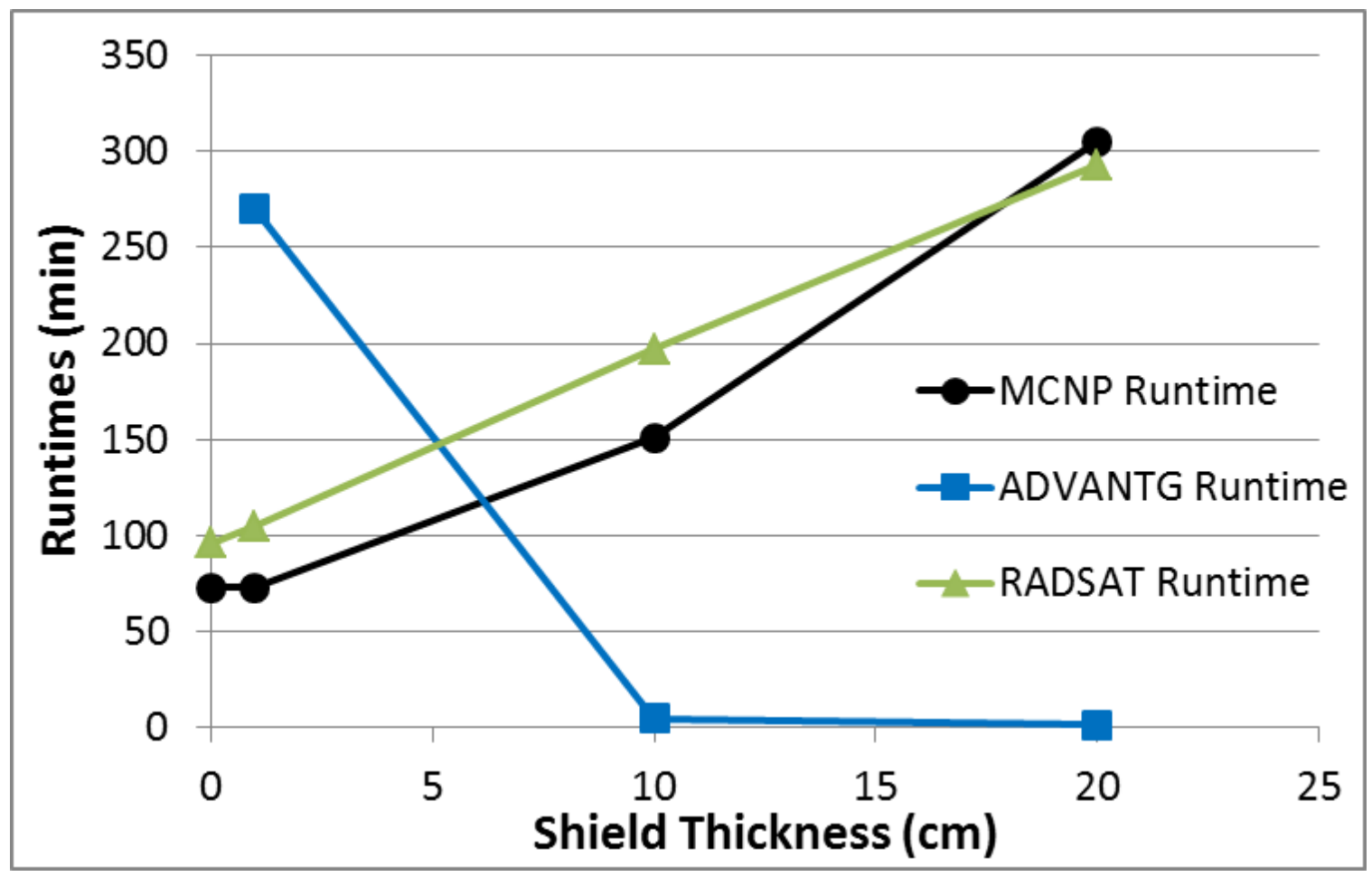

Figure 10 Runtime Comparisons for varying shielding thicknesses. 


\subsection{Mulch Box Case}

\subsection{Description}

This problem assumes a high performance germanium detector and a ${ }^{133} \mathrm{Ba}$ gamma source shielded by a wooden box containing low density mulch material. This problem represents a very basic shielding problem in gamma spectroscopy, with photon transport through approximately 1-2 mean free paths and requirements for preserving the energy structure of the source and computing a detector response function. The box rests on a slab of concrete with soil below as shown below in Figure 3.1. The experiment collected data over about 800 seconds. While this case has no experimental results, it is used as the gamma test case for verification and validation of the RADSAT software during its development and as a test case in the RADSAT tutorial [Smith, et al 2009]. The geometry and materials are selected to test the sufficiency of the approach to handle scattering and avoid spurious ray effects.

Another advantage of this test case is that the user can artificially dial up and down density of the mulch to change what physical process is most important to detector response. For example, with a low density mulch, most of the detector counts will go through the box and will be uncollided. At a medium density, counts will be contributed from uncollided photons traveling through the box, collided photons traveling through the box, and collided photons traveling around the box. At very high densities, the scattered photons will dominate the detector response.

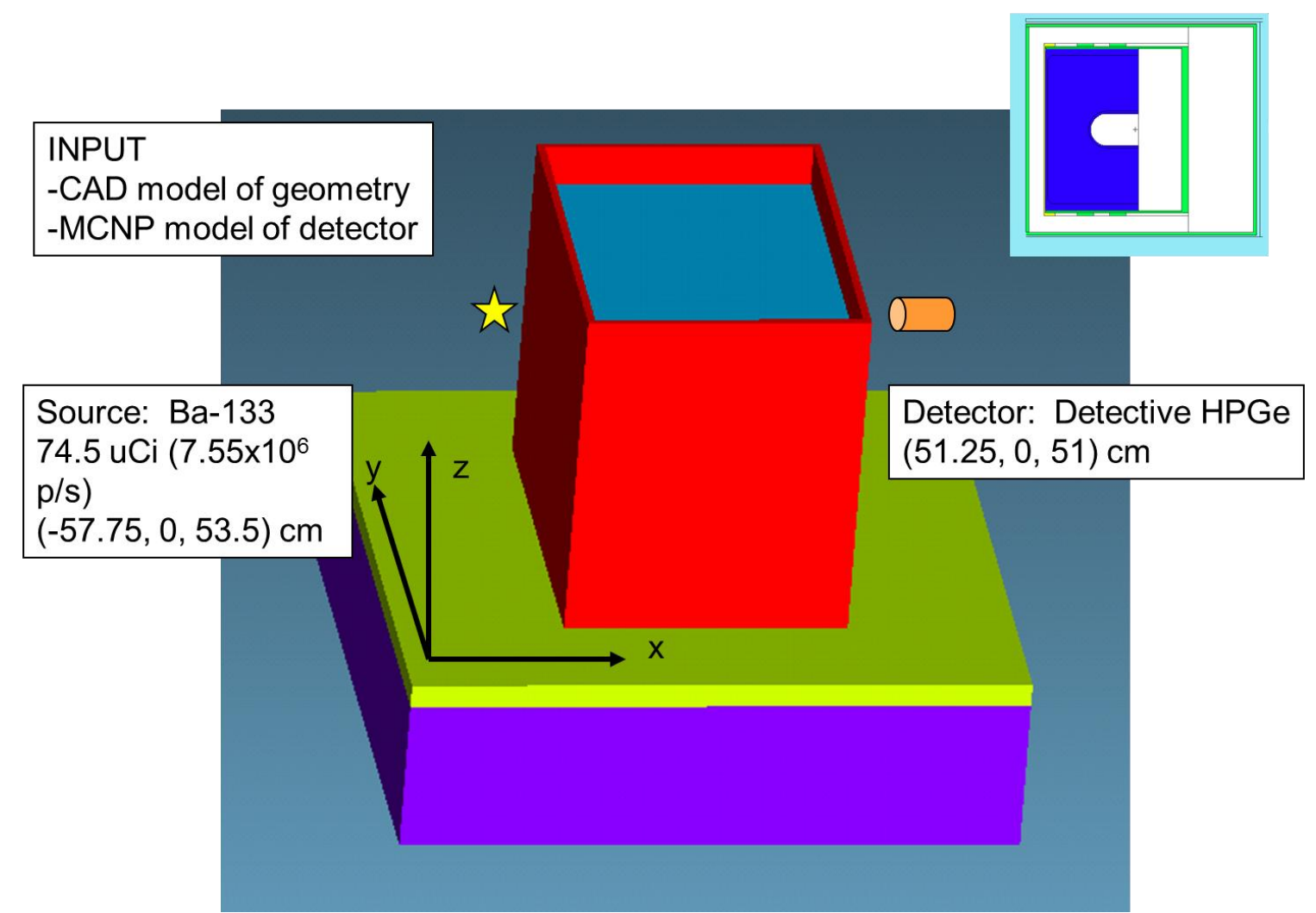

Figure 11. The geometry for the Mulch Box case with a detailed cutout of the MCNP model of the detector in the upper right hand corner. 


\subsection{Comparison of Results}

In RADSAT this case is run with S8P2 Triangle Chebychev Labotto quadrature and Galerkin scattering. The Attila case had a global mesh size of $0.3 \mathrm{~cm}$. For the ADVANTG run, all of the energy peaks converged faster than MCNP, but there were some issues with ray effects in the streaming problem of the adjoint calculation. The bulk transport in this case is a good example a configuration that is difficult for Monte Carlo methods and well suited for a forward deterministic calculation. While ADVANTG did speed up the Monte Carlo run, the pure deterministic transport of RADSAT was faster. Results shown below in Table 3.1 and Figure 3.2 .

Table 3.1. Summary of Results of peak counts, total counts, and runtime

\begin{tabular}{|c|c|c|c|c|c|c|c|}
\hline Method & $\begin{array}{c}\text { Total } \\
\text { (cps) }\end{array}$ & $\begin{array}{c}\mathbf{8 0 ~ k e V} \\
\text { (cps) }\end{array}$ & $\begin{array}{c}\mathbf{2 7 6 . 4 ~ k e V} \\
\text { (cps) }\end{array}$ & $\begin{array}{c}\mathbf{3 0 2 . 9} \mathbf{~ k e V} \\
\text { (cps) }\end{array}$ & $\begin{array}{c}\mathbf{3 5 6} \mathbf{~ k e V} \\
\text { (cps) }\end{array}$ & $\begin{array}{c}\mathbf{3 8 3 . 8} \text { keV } \\
\text { (cps) }\end{array}$ & $\begin{array}{c}\text { Run Time } \\
\text { (min) }\end{array}$ \\
\hline Experimental & 137 & 11.3 & 2.1 & 2.2 & 3.8 & 0.53 & 13.33 \\
\hline MCNP & 124 & 11.9 & 2.1 & 2.4 & 4.3 & 0.55 & 920 \\
\hline \% Difference & $-9.5 \%$ & $5.3 \%$ & $0.0 \%$ & $9.1 \%$ & $13 \%$ & $3.8 \%$ & $6,800 \%$ \\
\hline ADVANTG & 128 & 11.9 & 2.2 & 2.5 & 4.4 & 0.54 & 36 \\
\hline \% Difference & $-7.1 \%$ & $5.6 \%$ & $2.7 \%$ & $9.8 \%$ & $15 \%$ & $2.8 \%$ & $270 \%$ \\
\hline RADSAT & 121 & 10.5 & 2.0 & 2.2 & 3.8 & 0.56 & 9 \\
\hline$\%$ Difference & $-11.7 \%$ & $-7.08 \%$ & $-4.8 \%$ & $0.0 \%$ & $0.0 \%$ & $5.7 \%$ & $-32.5 \%$ \\
\hline
\end{tabular}

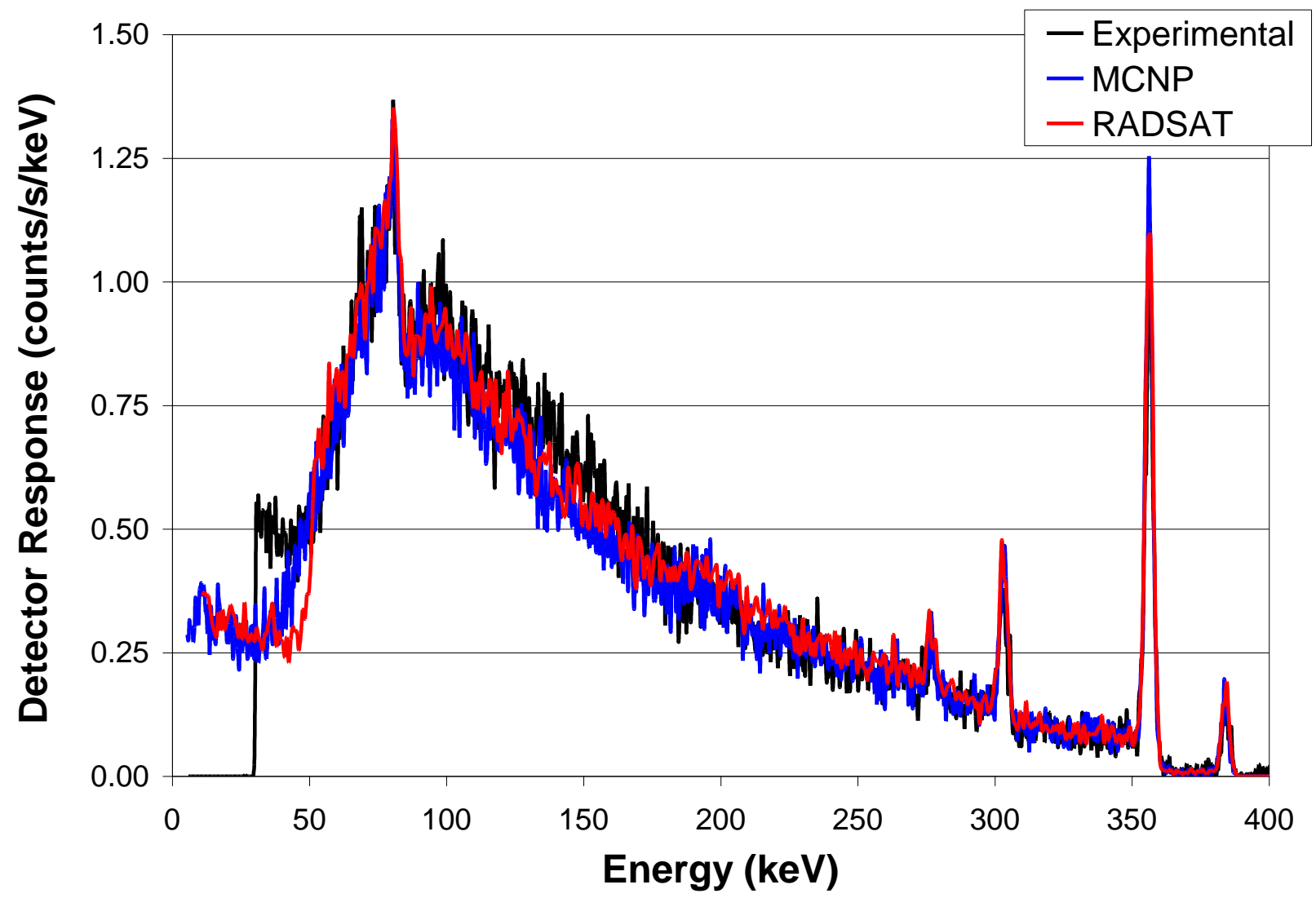

Figure 12. Comparison of Experiment, MCNP, and RADSAT 


\subsection{Skyshine Gamma Background Case}

\subsection{Description}

This case considers the large field backscatter of bremsstrahlung photons produced in soil from beta decay of ${ }^{40} \mathrm{~K},{ }^{235} \mathrm{U},{ }^{238} \mathrm{U}$, and ${ }^{232} \mathrm{Th}$ and their progeny. As a simulation problem, this represents a very large area scenario, with considerable scattering and long paths for transport (leading to a high sensitivity to particle angle). The purpose is to simulate the main contribution to low energy $(<500 \mathrm{keV})$ background observed in gamma detectors for radiation portal monitoring [Kernan, et al., 2009]. A visualization of the detector mounted $2.1 \mathrm{~m}$ above the ground on a small platform supported by a structure of PVC pipe is shown below in Figure 4.1. The air medium properties used in the calculations of temperature, relative humidity, and pressure are the measured values for time of the corresponding count-rate measurement: $T_{0}=$ $6.2^{\circ} \mathrm{C}, \mathrm{P}=1024.9-1005.82 \mathrm{mb}$. The air density is $0.00125 \mathrm{gm} / \mathrm{cc}$. These are consistent with the modeled composition of air.

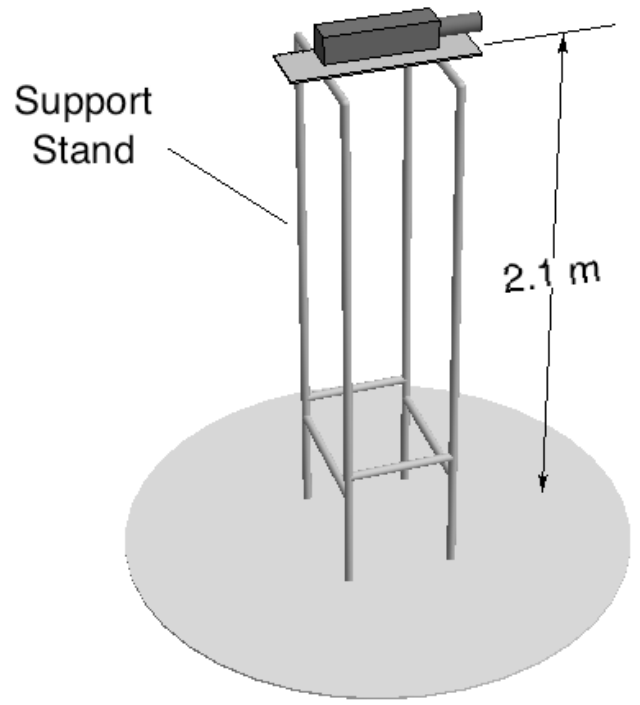

Figure 13. The geometry for the Skyshine Gamma Background Case.

\subsection{Comparison of Results}

In MCNP, the source is considered to be a 1,600 $\mathrm{m}$ radius disk surface source below a hemisphere of air with detectors represented on about a $50 \mathrm{~m}$ region shown below in Figure 4.2. The air volume is, therefore, $8.58 \mathrm{~km}^{3}$. The detector is modeled as a $9 \times 9$ array of $10.2-\mathrm{cm} \times$ 10.2-cm x 40.6-cm (4-in. x 4-in. x 16-in.) Nal(TI) detectors spaced $5 \mathrm{~m}$ apart shown below in Figure 4.3. For variance reduction the MCNP model assumes importance weighted hemispherical shells with weighting that varies by a factor of about 80 from the near-field to the far-field region. Additional statistical improvement is obtained by averaging over the 81 detector tallies. 


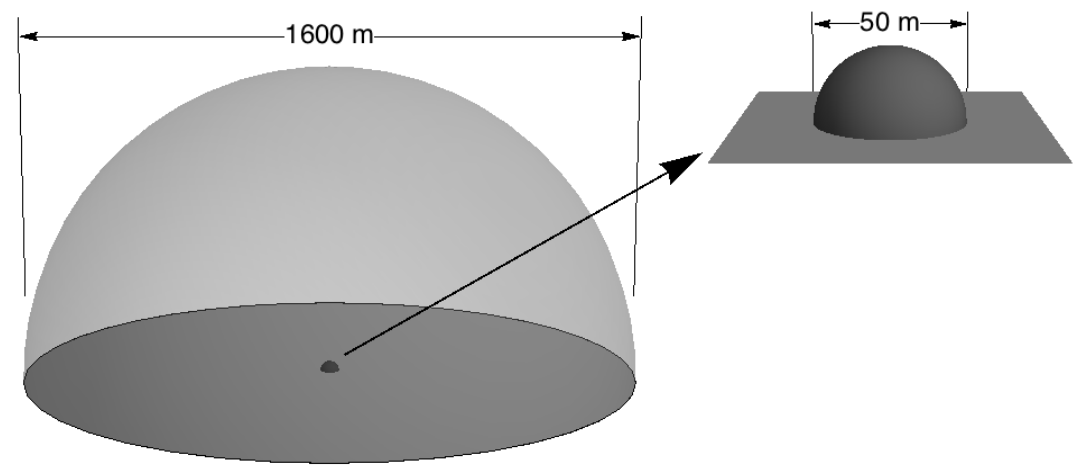

Figure 142. Configuration Used in MCNP to Calculate the Hemispherical Surface Source from a Disk Surface Source.

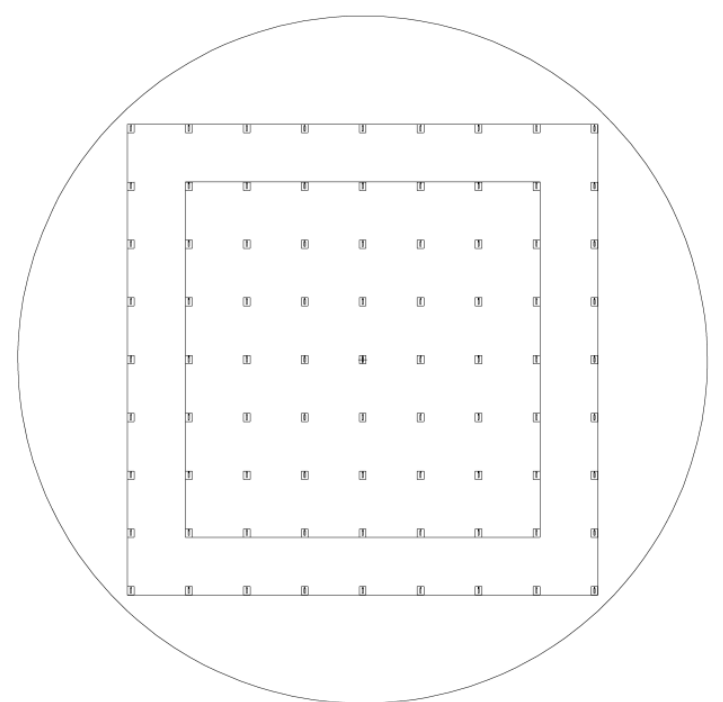

Figure 153. MCNP model Detector array region.

Figure 4.4 below shows the count rate plotted against the gamma-ray energy for a comparison of the calculated to measured gamma-ray background. The red curve is the sum of four 30 minute measurements. The blue curve is from the MCNP run of this case and is taken directly from the reference [Sandness, et al., 2011]. The output is for $10^{11}$ source particles. Because the source in this case is varied and has many features, instead of peak or total counts, a residuals plot is analyzed to look at the agreement of the calculation over all energy groups. The ADVANTG run yielded an essentially identical result to the MCNP run, but with a speedup of about a factor of 83 . 

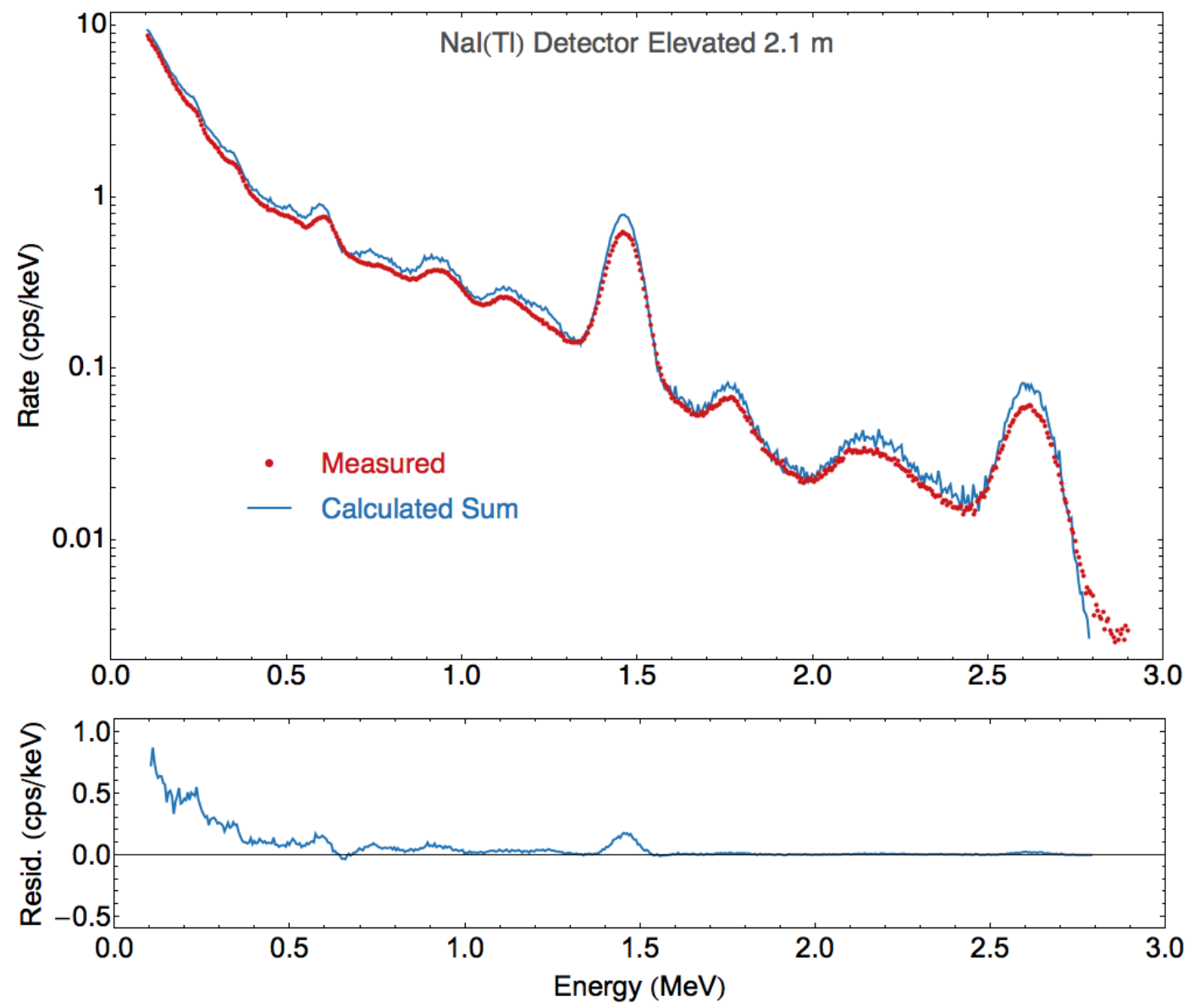

Figure 16. Comparison of the Calculated to Measured Gamma-Ray Background.

The geometry for the Attila model run is assumed to be a large block of air with a thin ground region containing the source. Figure 4.5 below shows the variable mesh scale at the bottom region of the cube of $500 \mathrm{~m}$ on each side, or a total volume of $0.125 \mathrm{~km}^{3}$, which is the maximum physical size allowed in Attila. There are no detectors present in the Attila model. The detector response is calculated with the angular flux solution at the detector position. The Attila model spatial mesh assumed about 360,000 cells with a variable mesh scale of $5 \mathrm{~m}$ near the ground to a mesh scale of $20 \mathrm{~m}$ in the air region. The angular resolution is P2 for the cross sections and S8 (Triangular Chebychev Legendre) for the transport quadrature. A total of 130 energy groups ranged from $0.25 \mathrm{MeV}$ to $3.3 \mathrm{MeV}$. On the same machine as the MCNP run the Attila solution for the angular flux throughout the geometry took about 2 hours. 


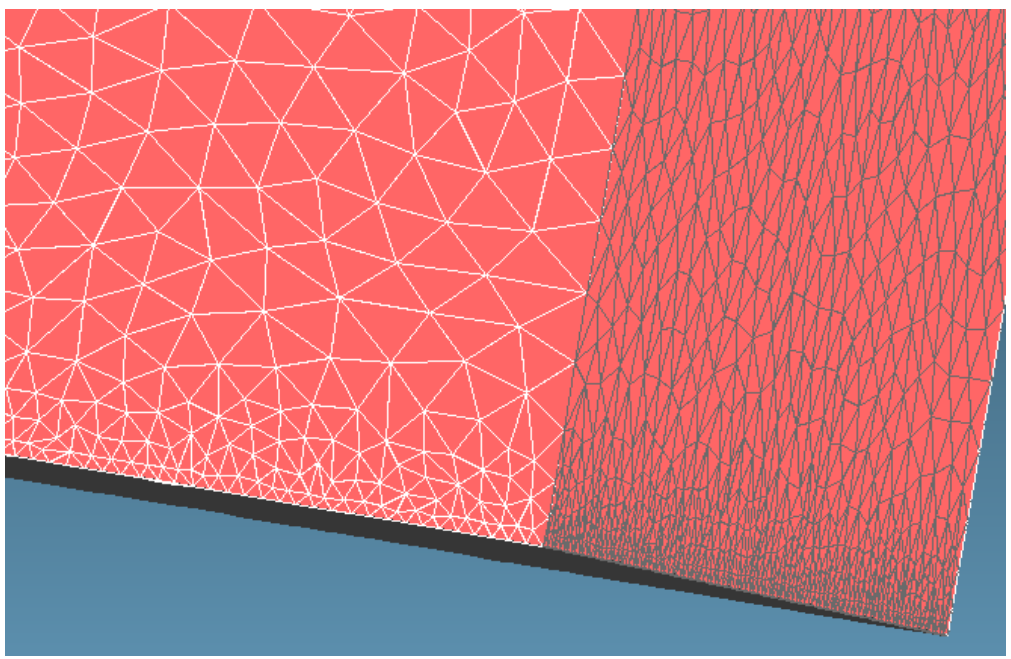

Figure 17. General tetrahedral mesh elements for air and ground.

A detector-only model, shown below in Figure 4.6 is built from the MCNP model and enclosed in an emission surface which reproduced the Attila calculated angular flux.

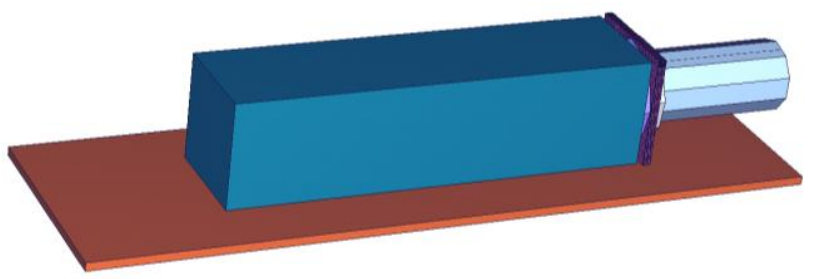

Figure 18. Single Detector used for Detector Response Function.

Figure 4.7 shows the results for detector counts. To achieve the same statistics as MCNP, the detector response function runs for about 6 hours on the same machine. As can be seen, the results have the same shape as the MCNP ad measured values, but are a much lower order of magnitude. Figure 4.7 shows the effect of the overall geometry size on the magnitude of the detector count rate. The volume of air in Attila is 68.6 times smaller than that in MCNP, so if Attila could have a volume that large (or using another deterministic code that can support a larger problem geometry), the runtime for Attila would be increased to around 138 hours and the detector response would still be 6 hours, making the total $\sim 144$ hours, as opposed to the $\sim 502$ hours for MCNP with variance reduction. 


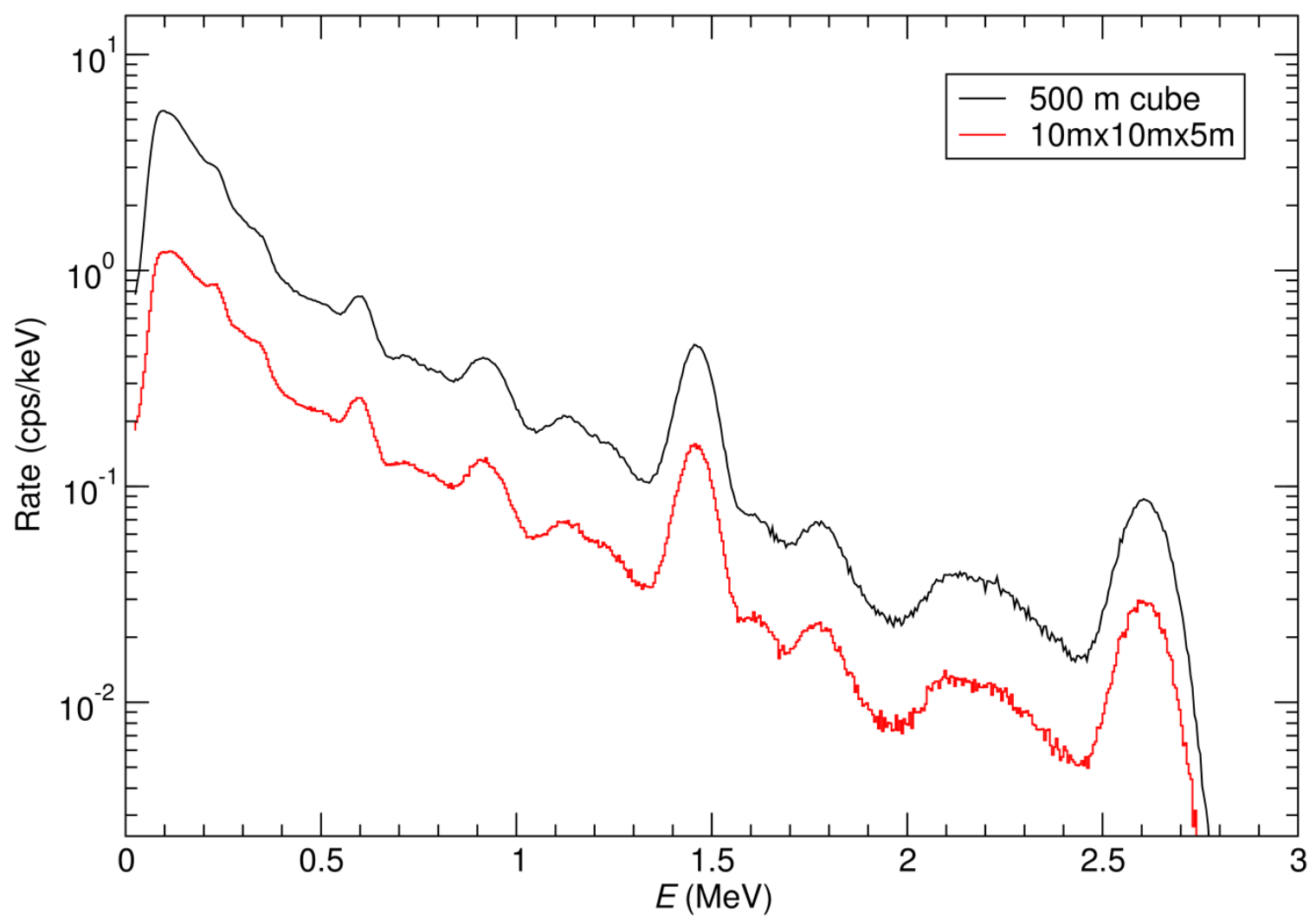

Figure 19. Results for Small field (red) and Large field (black) Attila Model in RADSAT.

Table 4.1. Comparison of Runtimes for the Sky Shine detector

\begin{tabular}{|c|c|}
\hline Method & Run Time (hours) \\
\hline INL - Experimental & 2 \\
\hline MCNPX (analog) & 3,687 \\
\hline Difference (\%) & $184,000 \%$ \\
\hline MCNPX (source energy biasing) & 502 \\
\hline Difference (\%) & $25,000 \%$ \\
\hline ADVANTG & 44 \\
\hline Difference (\%) & $2,110 \%$ \\
\hline RADSAT & 144 \\
\hline Difference (\%) & $7,100 \%$ \\
\hline
\end{tabular}




\subsection{Spent Fuel Cask Gamma-Ray Scanner}

\subsection{Description}

This case is a Spent Fuel Cask Gamma-Ray Scanner consisting of a highly collimated detector that measures the gamma-ray response above each spent fuel assembly location in the cask. This problem represents a considerably more complex scenario, with both gamma and neutron transport, a detailed and complex geometry, and a strongly collimated detector. The system was developed by Gus Caffrey and his team from INL. This case was documented previously in the RADSAT Test Case Implementation Final Report (Shaver 2010), as a test case of the RADSAT software to applications of interest to NA-22. A visualization of the scanner is shown below in Figure 5.1 and the pattern over which it moves to scan all locations is shown below in Figure 5.2. The combination of assembly self-shielding and the 14 inch steel lid (made to shield the radiation from the spent fuel to the outside) between the source and detector make this case very challenging to model and get accurate results.

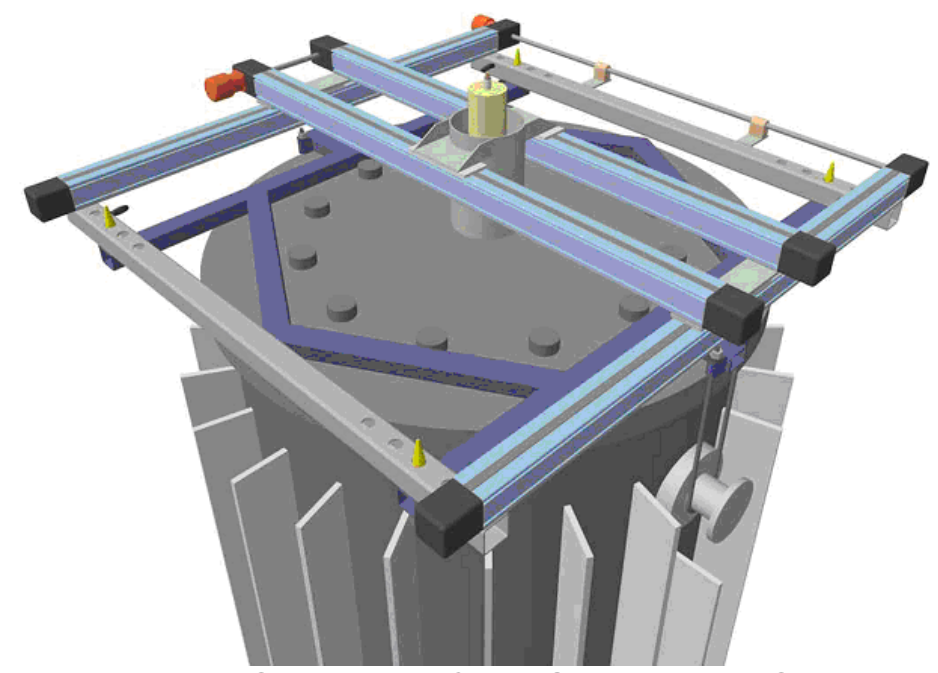

Figure 20. The geometry for the Spent Fuel Cask Gamma-Ray Scanner case [Caffrey 2009].

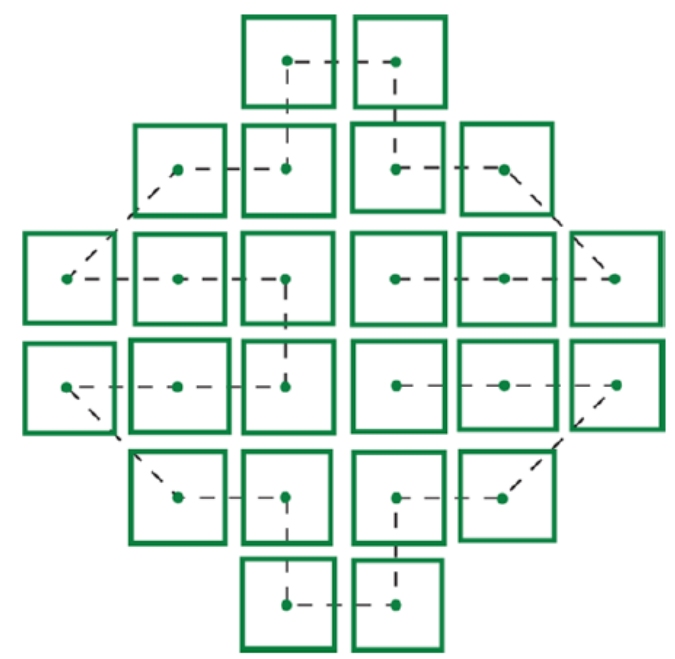

Figure 21. Scanning Pattern of a Westinghouse MC-10 Spent Fuel Cask [Caffrey 2009]. 
The detector is a HPGe $7.13 \times 5.86 \mathrm{~cm}$ cylinder (dark blue in Figure 5.3) with 8,000 channels. The purple region in the figure is the collimator, while the dark gray is the shielding. The specific scenario and experimental data was provided by INL is for Turkey Point Nuclear Reactor spent fuel in a Westinghouse MC-10 spent fuel cask. A diagram of the cask and assembly locations is shown below in Figure 5.4. The two assembly positions that are modeled for comparison are the full $\mathrm{C} 2$ and empty A4 slots. These positions are indicated by being circled on in Figure 5.4 and the position of the detector is directly over the circled assemblies. The source is an array of 30 year decayed PWR fuel assemblies.

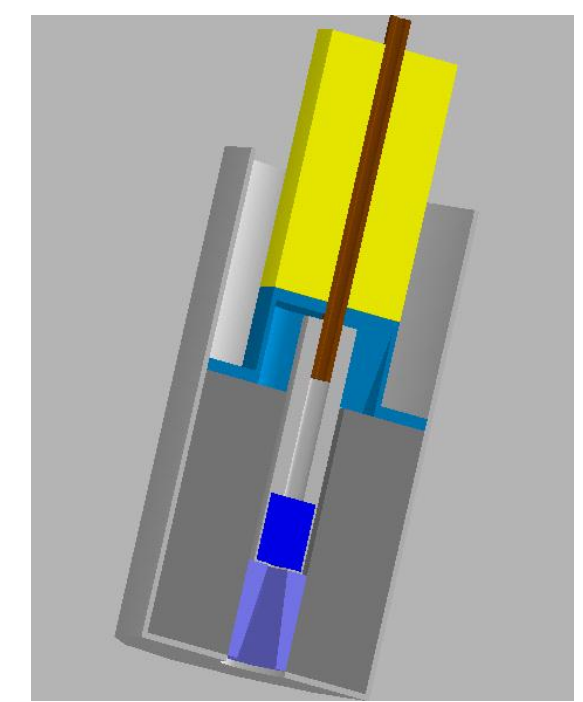

Figure 22. MCNP detector model.

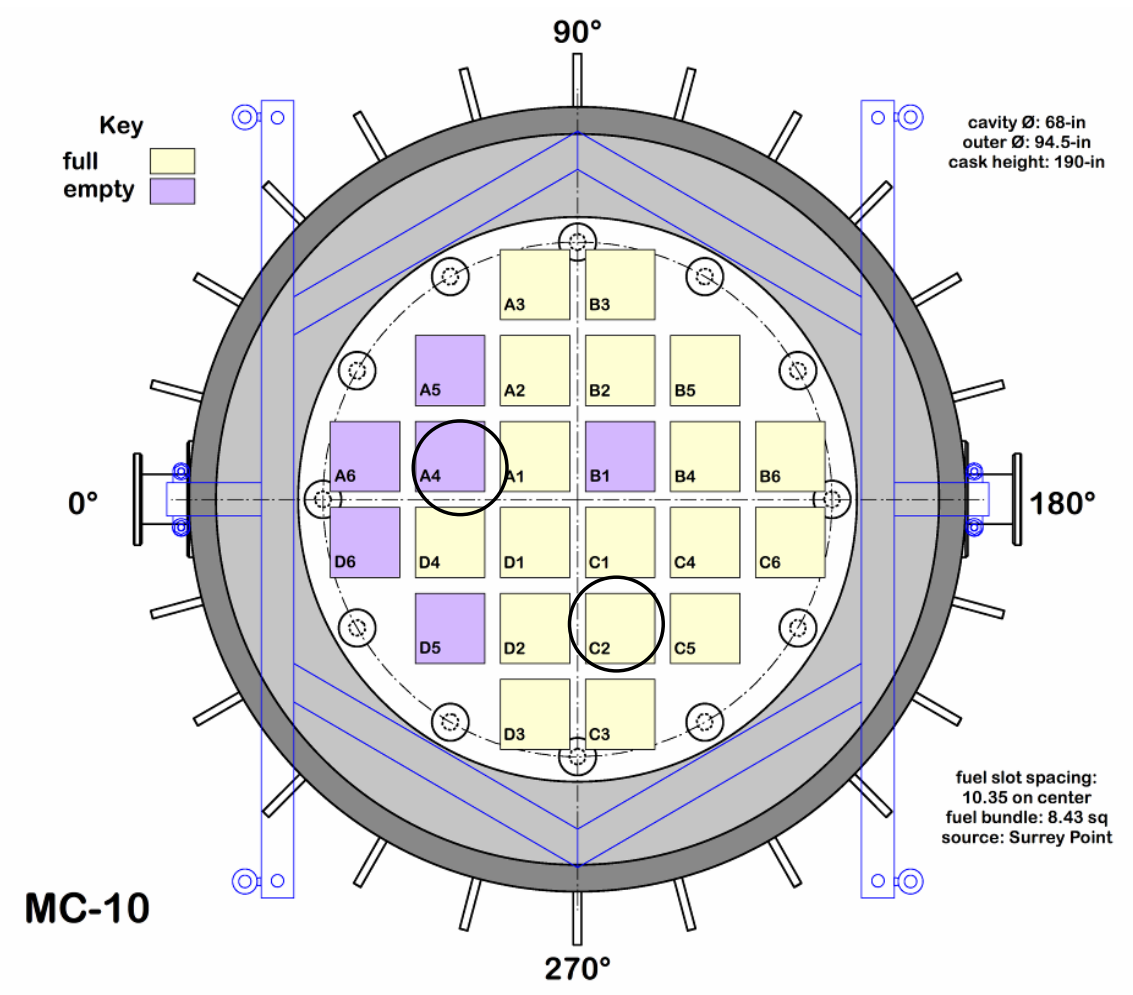

Figure 23. Configuration and loading of the Turkey Point spent fuel cask [Caffrey 2009]. 


\subsection{Comparison of Results}

The fuel assemblies are modeled as the homogeneous average of source and materials. For Monte Carlo runs, the 1,000 second live-time spectral measurement each with assembly source activities of about $10^{15}$ gammas per second would require the tracking of about $10^{20}$ source particles. No $n, y$ reactions are modeled as they were previously determined to not be important. To get around much of this difficulty, the Monte Carlo N-Particle eXtended (MCNPX) [Waters et al. 2002] model made by INL to evaluate the effectiveness of the detection system has multiple variance reduction techniques in it. First, due to self-shielding of the assemblies, only the top 75 $\mathrm{cm}$ of the fuel is modeled. Next, there is source biasing in the upward direction toward the scanner. Finally, there are weight windows in sections of the cask lid to increase the gamma importance as the location becomes closer to the detector. A visualization of the MCNP geometry and weight windows for variance reduction is shown below in Figure 5.5. The ADVANTG runs were much faster than either of the other two methods for both of these cases. This is a case where the hybrid methodology is clearly the fastest way to solve the problem.

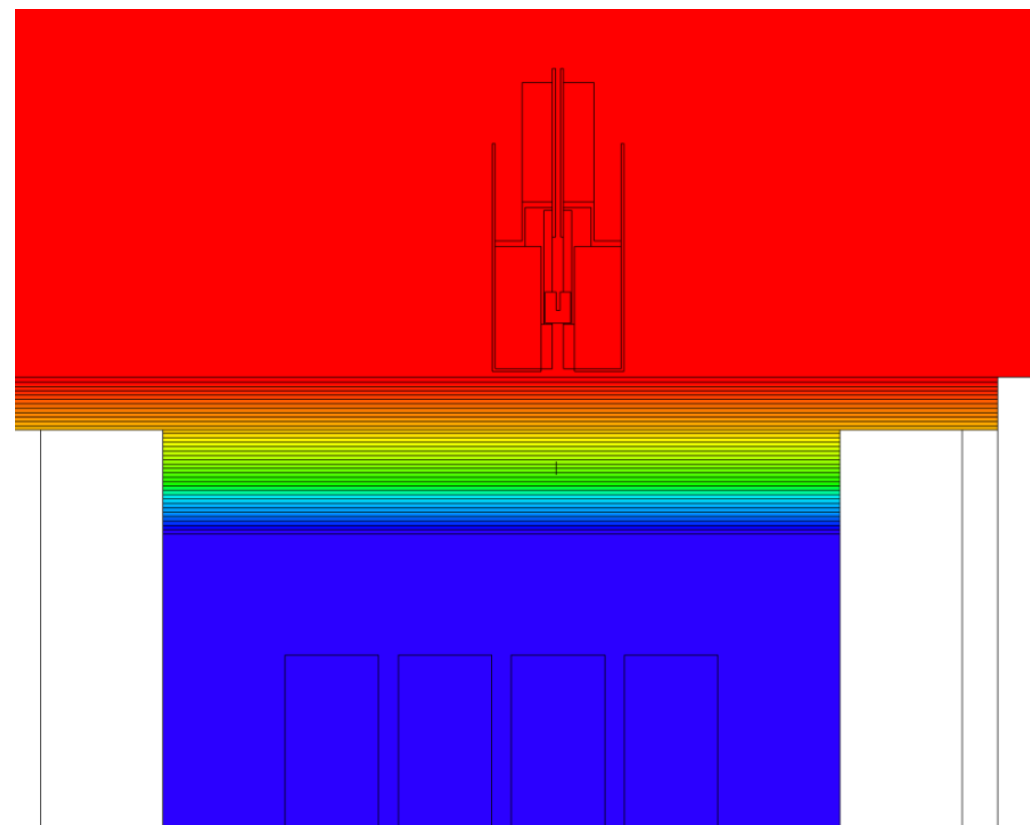

Figure 24. MCNP Gamma-Ray Spent Fuel Cask Scanner Model showing region of lowest (blue) to highest (red) importance used for variance reduction.

The MCNP model of the detector as shown above is used for both the RADSAT and MCNPX simulations. For the RADSAT simulation of the spent fuel cask scanner scenario, the mesh is meshed to a tightness of $0.1 \mathrm{~m}$ everywhere in the problem except for the lid, which is meshed to $0.05 \mathrm{~m}$. The cross section library is 101 groups and generated by the CEPXS module of RADSAT. The maximum outer and inner iterations are both 100, and the convergence criterion is 1E-4. The quadrature used is Triangle Chebychev Lobatto, with S8P2, and Galerkin Scattering turned on. A point source flux edit is taken by space and energy at points centered above each assembly. A visualization of the solid model for the RADSAT case is shown below in Figure 5.6. 

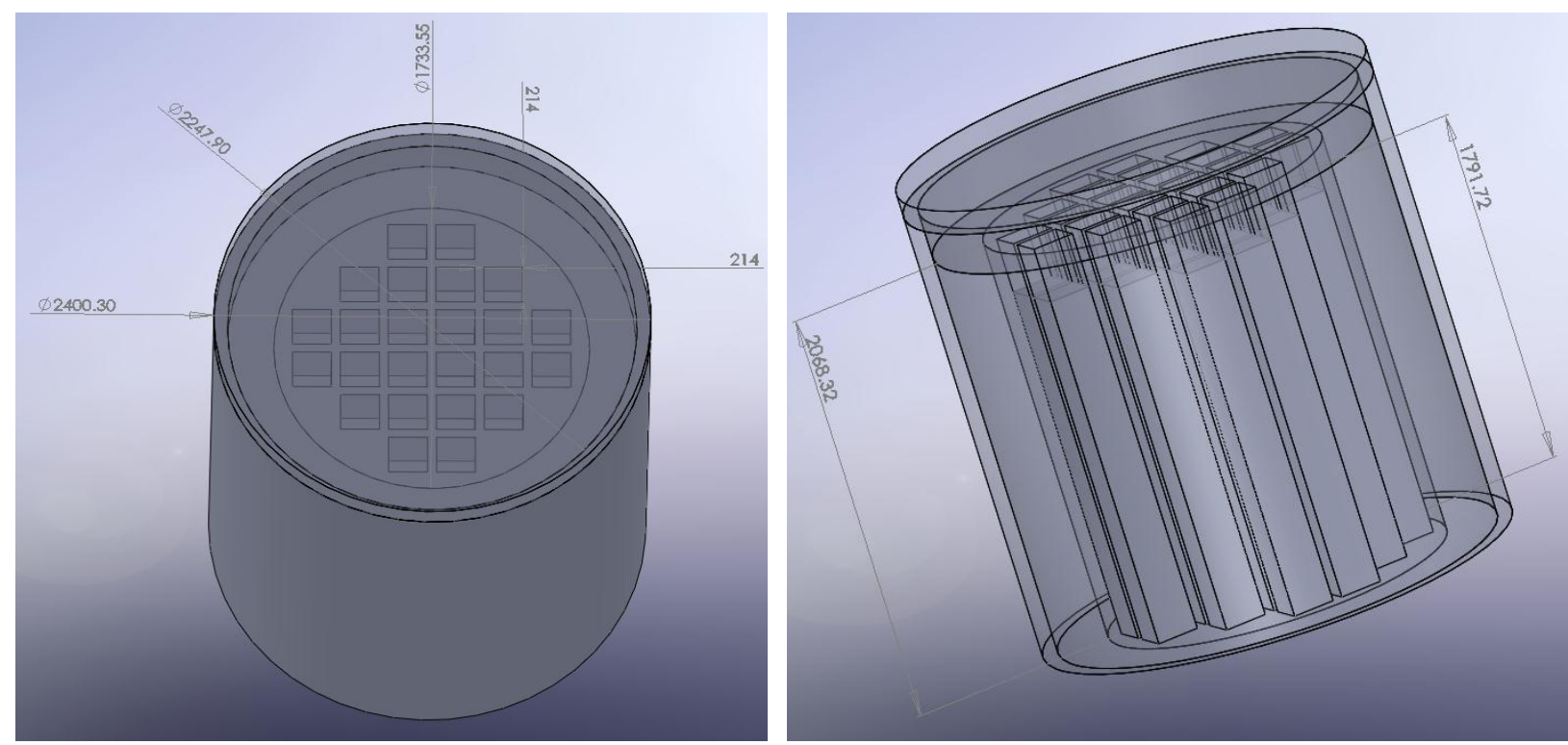

Figure 25. Solid Works CAD model of the spent fuel in the cask used for the Attila geometry

The spectral results of the experimental data, MCNPX and RADSAT for the full C2 assembly position are shown for comparison below in Figure 5.7. For analysis of the spent fuel, INL looks at the spectra between $700 \mathrm{keV}$ and $1100 \mathrm{keV}$ as well as certain peaks from different isotopes.

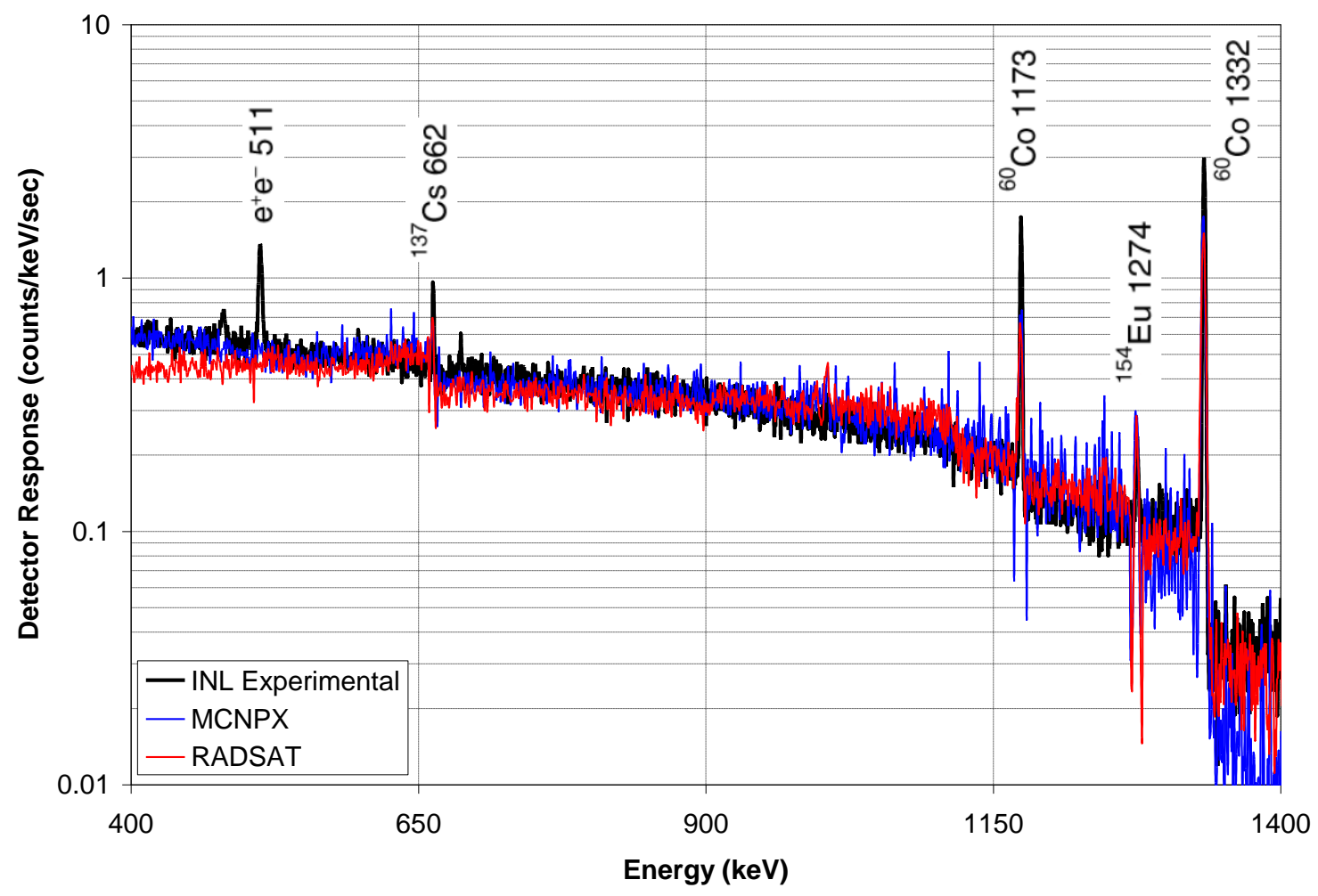

Figure 26. Comparison of Cask Scanner Detector Response in the full C2 slot. 
A comparison of the two simulations methods is compared to the experimental data in Table 5.1 below. The comparison metric is the integrated area under the energy region for the spectra and the integrated area under the peaks for the discrete isotope lines. As can be seen MCNPX and RADSAT yield consistent results with each other and with the experimental data, less than $15 \%$ deviation from experimental in all cases. In terms of run time, it took MCNPX over two days to simulate the 1,000 minutes of detector live time and RADSAT took just over one day to simulate the same detector live time. This means that RADSAT took less than $60 \%$ of the time of MCNPX to yield comparable answers. Both codes have been optimized for the best run time, including the MCNPX variance reduction mentioned previously.

Table 5.1. Comparison of Results for the Cask Scanner in the full C2 slot

\begin{tabular}{|c|c|c|c|c|c|c|}
\hline & $\begin{array}{c}\mathbf{7 0 0 - 1 , 1 0 0} \\
\text { keV (\%) }\end{array}$ & $\begin{array}{c}\mathbf{6 6 2} \mathbf{~ k e V} \\
\text { Peak (\%) }\end{array}$ & $\begin{array}{c}\text { 1173 keV } \\
\text { Peak (\%) }\end{array}$ & $\begin{array}{c}\mathbf{1 2 7 4} \text { keV } \\
\text { Peak (\%) }\end{array}$ & $\begin{array}{c}\text { 1332 keV } \\
\text { Peak (\%) }\end{array}$ & $\begin{array}{c}\text { Run Time } \\
\text { (min) }\end{array}$ \\
\hline INL - Experimental & - & - & - & - & - & 1,000 \\
\hline MCNPX & $14.3 \%$ & $14.7 \%$ & $-6.77 \%$ & $5.72 \%$ & $-8.20 \%$ & $\begin{array}{c}2,916 \\
191.6 \%\end{array}$ \\
\hline ADVANTG & $14.3 \%$ & $14.7 \%$ & $-6.77 \%$ & $5.72 \%$ & $-8.20 \%$ & $-97.1 \%$ \\
\hline RADSAT & $11.9 \%$ & $14.1 \%$ & $-10.6 \%$ & $-14.2 \%$ & $-10.5 \%$ & $68.7 \%$ \\
\hline
\end{tabular}

The spectral results of the experimental data, MCNPX and RADSAT for the empty A4 assembly position are shown for comparison below in Figure 5.8. For analysis of the spent fuel, INL looks at the spectra between $700 \mathrm{keV}$ and $1100 \mathrm{keV}$ as well as certain peaks from different isotopes.

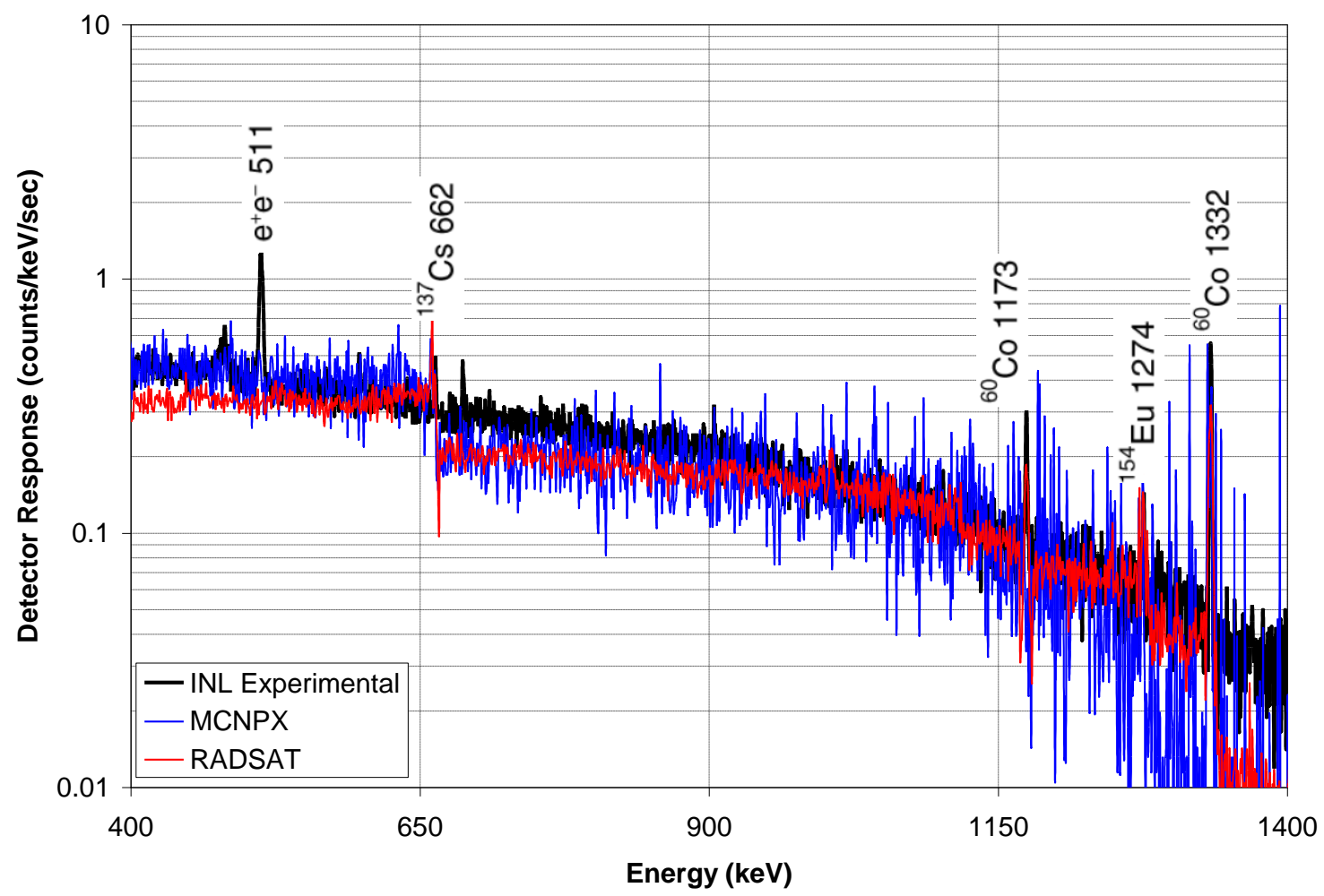

Figure 27. Comparison of Cask Scanner Detector Response in the empty A4 slot. 
A comparison of the two simulations methods is compared to the experimental data in Table 5.2 below. The comparison metric is the integrated area under the energy region for the spectra and the integrated area under the peaks for the discrete isotope lines. As can be seen MCNPX and RADSAT yield consistent results with each other and with the experimental data, less than $15 \%$ deviation from experimental in all cases. In terms of run time, it took MCNPX over two days to simulate the 1,000 minutes of detector live time and RADSAT took just over one day to simulate the same detector live time. This means that RADSAT took less than $60 \%$ of the time of MCNPX to yield comparable answers. Both codes have been optimized for the best run time, including the MCNPX variance reduction mentioned previously.

Table 5.2. Comparison of Results for the Cask Scanner in the empty A4 slot

\begin{tabular}{|c|c|c|c|c|c|c|}
\hline & $\begin{array}{c}\mathbf{7 0 0 - 1 , 1 0 0} \\
\text { keV (\%) }\end{array}$ & $\begin{array}{c}\mathbf{6 6 2} \mathbf{~ k e V} \\
\text { Peak (\%) }\end{array}$ & $\begin{array}{c}\mathbf{1 1 7 3} \mathbf{k e V} \\
\text { Peak (\%) }\end{array}$ & $\begin{array}{c}\mathbf{1 2 7 4} \mathbf{k e V} \\
\text { Peak (\%) }\end{array}$ & $\begin{array}{c}\text { 1332 keV } \\
\text { Peak (\%) }\end{array}$ & $\begin{array}{c}\text { Run Time } \\
\text { (min) }\end{array}$ \\
\hline INL - Experimental & - & - & - & - & - & 1,000 \\
\hline MCNPX & $2.09 \%$ & $12.3 \%$ & $-4.23 \%$ & $-14.5 \%$ & $1.44 \%$ & $2.1 \%$ \\
\hline ADVANTG & $2.09 \%$ & $12.3 \%$ & $-4.23 \%$ & $-14.5 \%$ & $1.44 \%$ & $-75 \%$ \\
\hline RADSAT & $-0.811 \%$ & $10.9 \%$ & $13.3 \%$ & $10.1 \%$ & $-1.59 \%$ & $-0.8 \%$ \\
\hline
\end{tabular}

Comparing the two assembly position cases (the full $\mathrm{C} 2$ slot and the empty A4 slot) produces Figure 5.9 below. It is apparent that both MCNPX and RADSAT faithfully represent the difference in magnitude in the spectra between the full and empty locations in the cask.

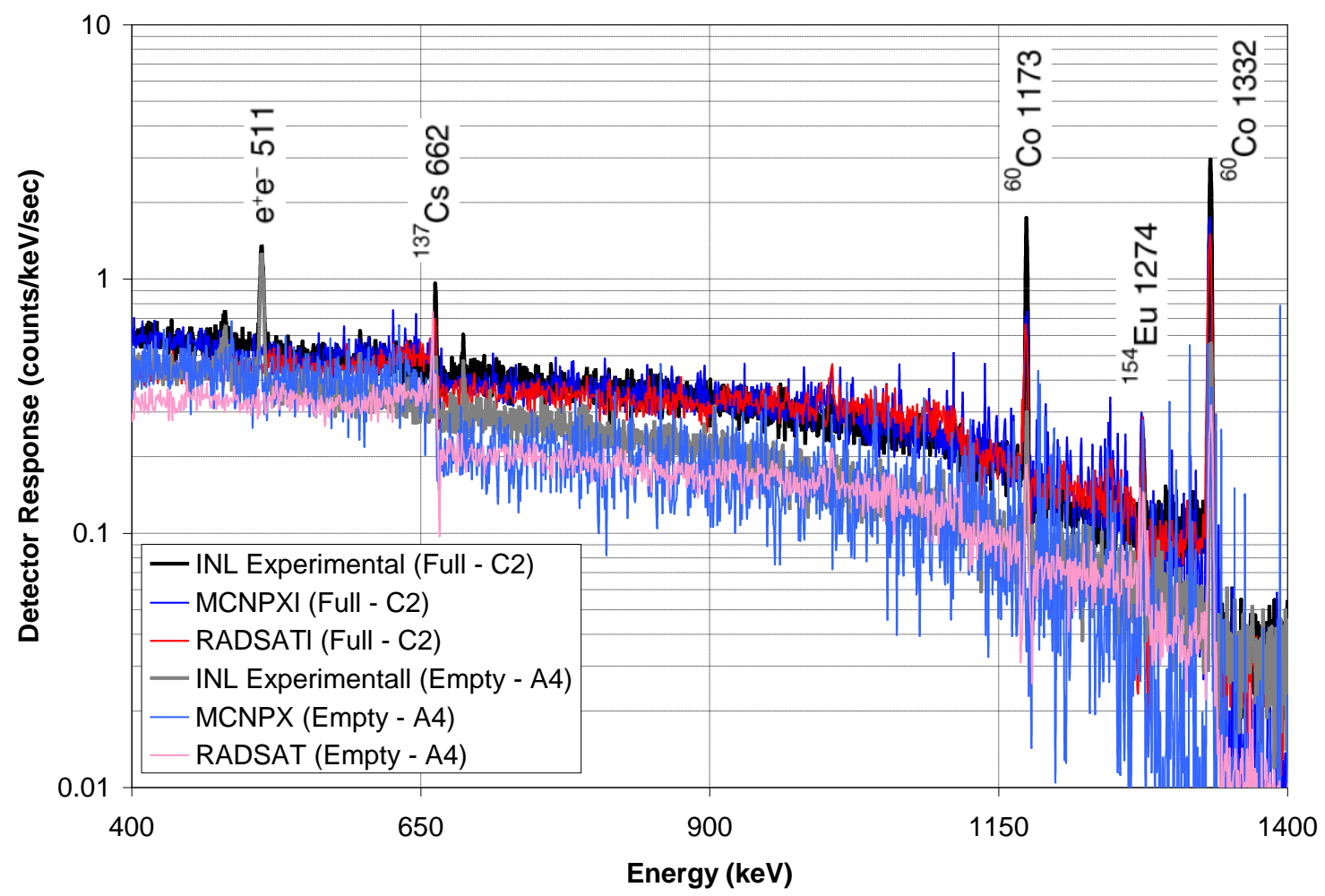

Figure 28. Comparison of Cask Scanner Detector Response in the full C2 and empty A4 slots 
It is important to note that there are certain features in the simulated data that are not apparent in the experimental data and vice versa. The $511 \mathrm{keV}$ peak does not show up in the simulated data because the positron annihilation after pair production is not modeled in the Monte Carlo simulations. Therefore, this peak does not show up in either MCNPX or RADSAT. Also, it is quite common for simulated gamma-ray detector responses show fewer counts than experimental data in the energies just higher than a peak. Again this feature is apparent for both MCNPX and RADSAT. Lastly, the peak heights do not completely match up in the simulated data because of the Gaussian energy broadening terms not being quite the same as the measured data. The metric compared is the integrated counts under each of the peaks or the region of interest. 


\subsection{Generic Radiation Portal Monitor Case}

\subsection{Description}

Another case extensively modeled by PNNL is the Radiation Portal Monitor. This could be both a neutron and gamma case as there are both neutron and gamma detectors, but only gamma detection is included here. This represents a photon transport problem, with spectroscopic detectors located over a range of positions. This case is a PNNL generic advanced spectroscopic portal (PGASP) monitor. The four Nal gamma detectors in this model are taken to be identical, individually (lead) shielded, 4"W x16"H x2"D crystals, positioned in a two-by-two array spaced vertically to span the full height available within each panel as shown below in Figure 6.1. A single panel is representative of PNNL RPM prototype detector system which is tested with various sources.

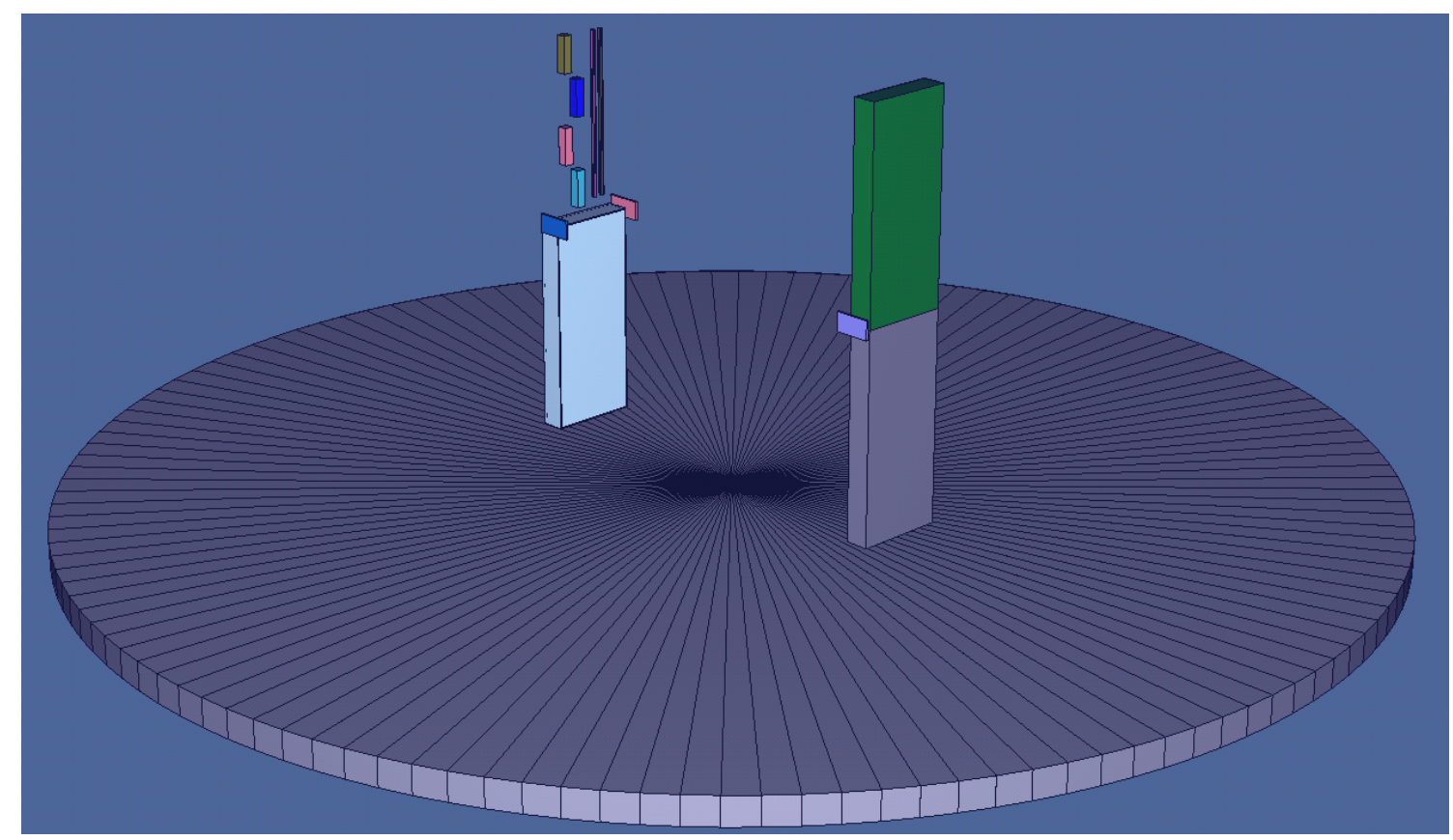

Figure 29. The geometry for the Generic Radiation Portal Monitor case.

\subsection{Comparison of Results}

The detector measurements are made over 5 minutes for sources at a distance of $2 \mathrm{~m}$ from the front of the panel door. Detector counts for a ${ }^{60} \mathrm{Co}$ source are used to calibrate the 1024 energy channels. Calibration and comparisons assume background subtracted data. Figure 6.2 and Table 6.1 show the comparison of the MCNP model results and the background subtracted data. The background is shown to highlight that the results in the low energy region may be farther from the calculation values, at least in part because of the large background subtraction. Also, because of this the total counts may not compare well, but the peak counts, which are what is important, do compare well (within $10 \%$ of experimental). The ADVANTG calculations yielded essentially identical answers in about $10 \%$ of the runtime. 


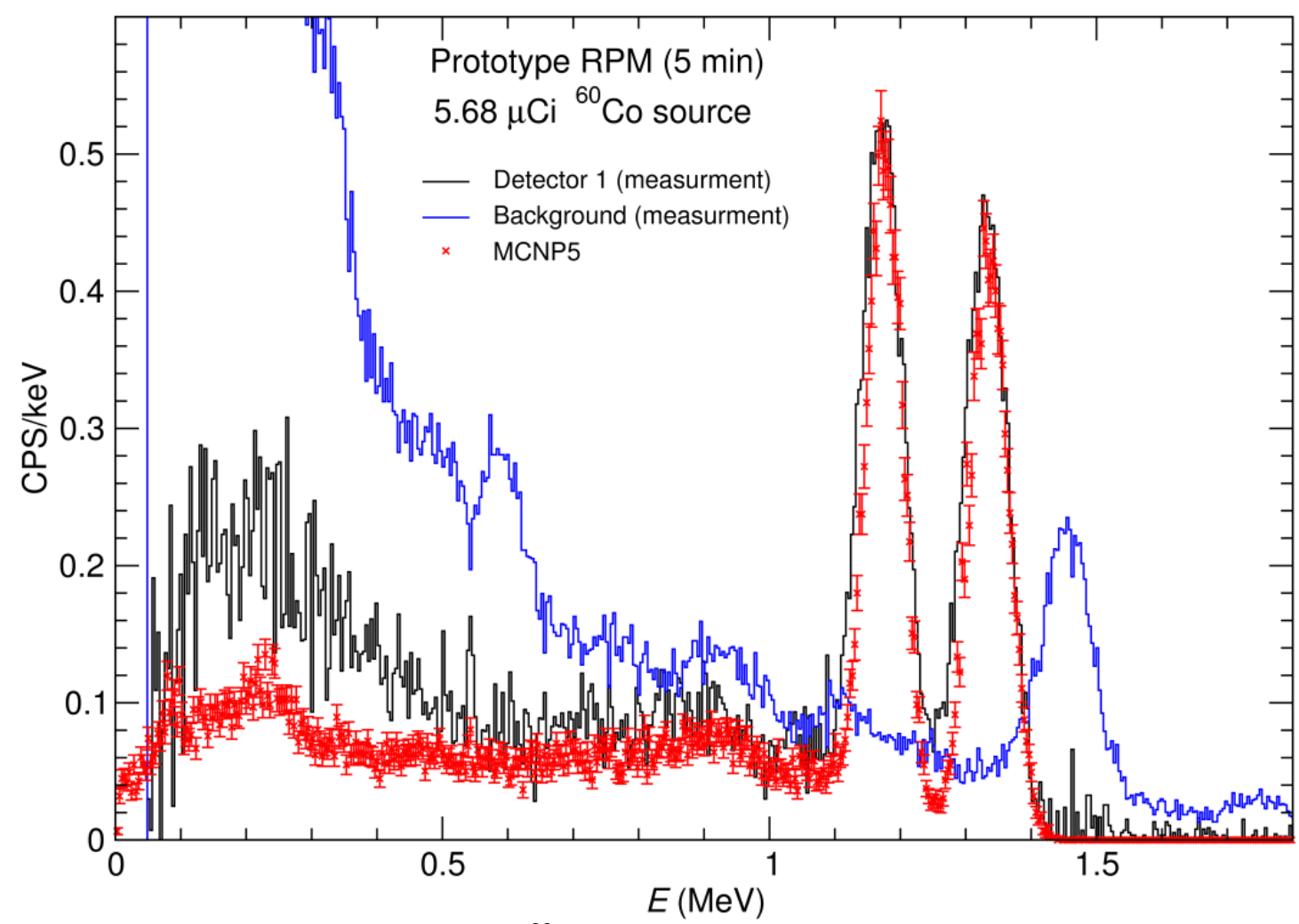

Figure 30. Comparison of MCNP with ${ }^{60} \mathrm{Co}$ source measurements for data used as calibration.

Table 6.1. Summary of Results for ${ }^{60} \mathrm{Co}$

\begin{tabular}{|c|c|c|c|c|}
\hline Method & $\begin{array}{c}\text { Total } \\
\text { (cps) }\end{array}$ & $\begin{array}{c}1173 \mathbf{~ k e V} \\
\text { (cps) }\end{array}$ & $\begin{array}{c}\mathbf{1 3 3 2} \mathbf{~ k e V} \\
\text { (cps) }\end{array}$ & $\begin{array}{c}\text { Run Time } \\
\text { (min) }\end{array}$ \\
\hline Experimental & 211 & 40 & 35 & - \\
\hline MCNP & 144 & 36 & 32 & 13.0 \\
\hline Diff (\%) & $-31.8 \%$ & $-10 \%$ & $-8.6 \%$ & - \\
\hline ADVANTG & 144 & 36 & 32 & 1.3 \\
\hline Diff (\%) & $0.0 \%$ & $0.0 \%$ & $0.0 \%$ & $-90 \%$ \\
\hline
\end{tabular}

The next case is a $10 \mu \mathrm{Ci}{ }^{137} \mathrm{Cs}$ source, but the age of the source is unknown. The results are shown below in Figure 6.3 and Table 6.2. The peak is reasonable for the position, but the height and width are in less agreement with the data. It is possible that some of the disagreement is from the uncertainty in the source activity. The decayed activity is assumed from other measurements made around the same time period, but it is not possible to confirm that these are precisely the same sources. Also, the widths of the peaks for both ${ }^{60} \mathrm{Co}$ and ${ }^{137} \mathrm{Cs}$ are slightly smaller in the modeled than the measured - this is likely because the GEB (Gaussian energy broadening) parameter in the MCNP model where not re-fit to the specific Nal crystals of this particular detector. The ADVANTG calculations yielded essentially identical answers in about $10 \%$ of the runtime. 


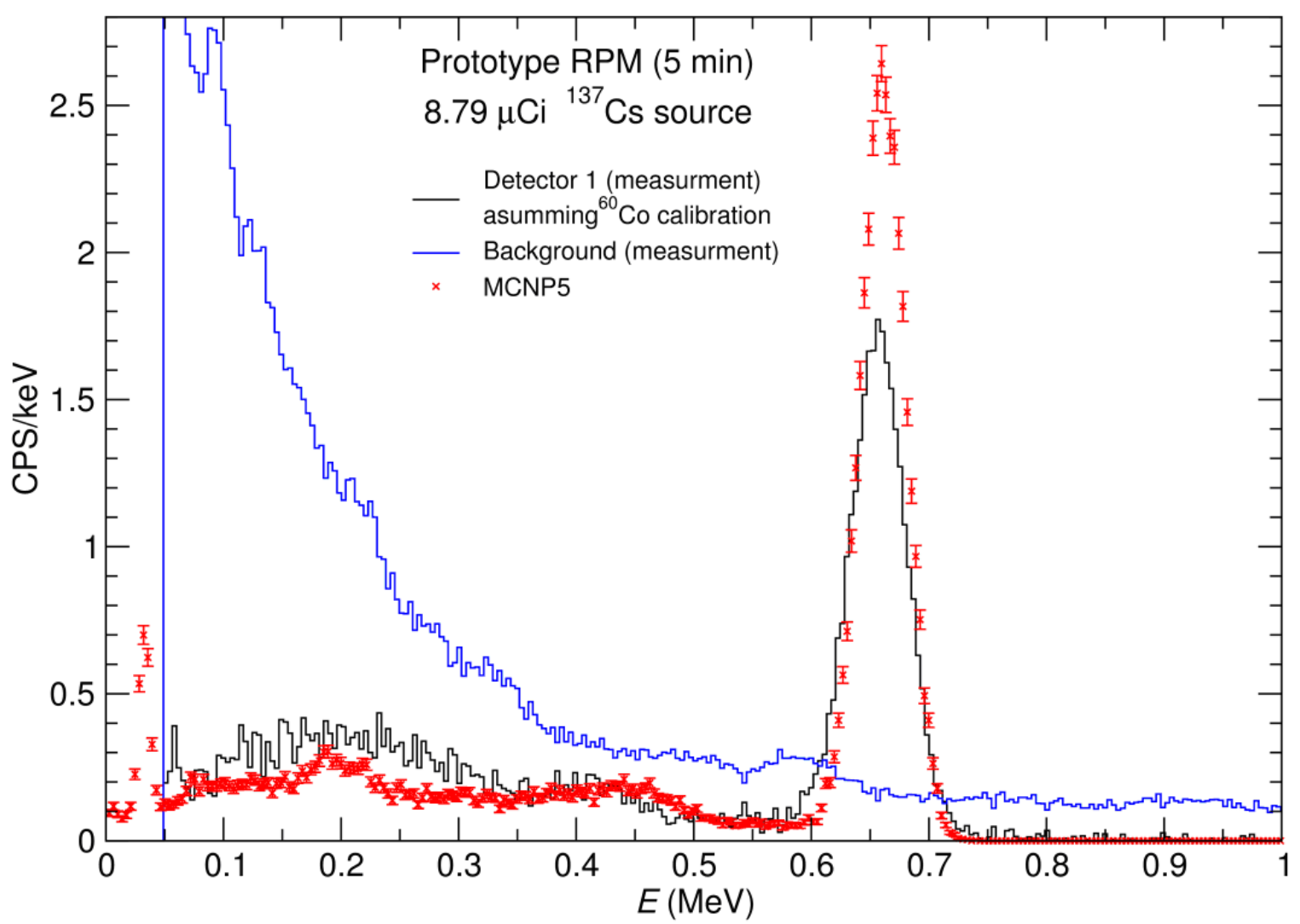

Figure 31. Comparison of MCNP with ${ }^{137} \mathrm{Cs}$ source measurements.

Table 6.2. Summary of Results for ${ }^{137} \mathrm{Cs}$

\begin{tabular}{|c|c|c|c|}
\hline Method & $\begin{array}{c}\text { Total } \\
\text { (cps) }\end{array}$ & $\begin{array}{c}662 \mathrm{keV} \\
\text { (cps) }\end{array}$ & $\begin{array}{c}\text { Run Time } \\
\text { (min) }\end{array}$ \\
\hline Experimental & 221 & 109 & - \\
\hline MCNP & 210 & 123 & 22.9 \\
\hline Diff (\%) & $-5.0 \%$ & $12.8 \%$ & - \\
\hline ADVANTG & 210 & 123 & 2.22 \\
\hline Diff (\%) & $0.0 \%$ & $0.0 \%$ & $-90 \%$ \\
\hline
\end{tabular}




\subsection{Radiography Case}

\subsection{Description}

This case represents a radiographic scan of a cargo container containing metal plates [Miller, et al., 2011]. It is included because it is much different than the standard detection case, but still of importance to scenarios of interest to NA-22. As a gamma transport problem, this case is both highly collimated (so strongly dependent on directionality) and requires high spatial resolution at the detector; radiography problems in general can include relatively high photon flux. The model consists of four major components: cargo truck, cargo (steel plate test frame), detector (on the radiography truck), and ${ }^{60} \mathrm{Co}$ source. The cargo truck consists of a truck bed modeled as a plate of $1-\mathrm{cm}$ thick carbon steel underneath a 2.54-cm-thick sheet of wood. Support beams are modeled beneath the steel truck bed in both lateral directions. The center of the cargo truck is located midway between source and detector. The radiography truck model consists of a steel plate for the truck bed, with two equipment boxes on either side of the detector housing (homogenized steel at a density of $1.0 \mathrm{~g} / \mathrm{cm}^{3}$ ), an $1800 \mathrm{~kg}$ steel counterweight, and a vertical support for the crane arm, which suspends the source and collimator. No tires are included on either truck. The ground is modeled as a cylindrical slab of concrete (18-meter diameter; $20-\mathrm{cm}$ thick); the surrounding environment is air with $50 \%$ relative humidity.

The ${ }^{60} \mathrm{Co}$ source rests inside a tungsten collimator. The opening angle is set to 3.21 horizontally (corresponding to an opening $0.84 \mathrm{~cm}$ wide) and 331 vertically. The vertical opening angle, in combination with measured source height and distance from the detector array, prevents the beam from shining directly on the ground. The source is modeled with an activity of $0.5 \mathrm{Ci}$. The geometry is shown below in Figure 7.1. The top is a side view of the model, with source and collimator at left and line detector on radiography truck at right. The gray fan represents the directly illuminated region. The bottom of the figure is a view of the radiography detector system looking down from above. The fan of incident radiation is perpendicular to the plane of the picture, with the source off screen at the bottom of the image.

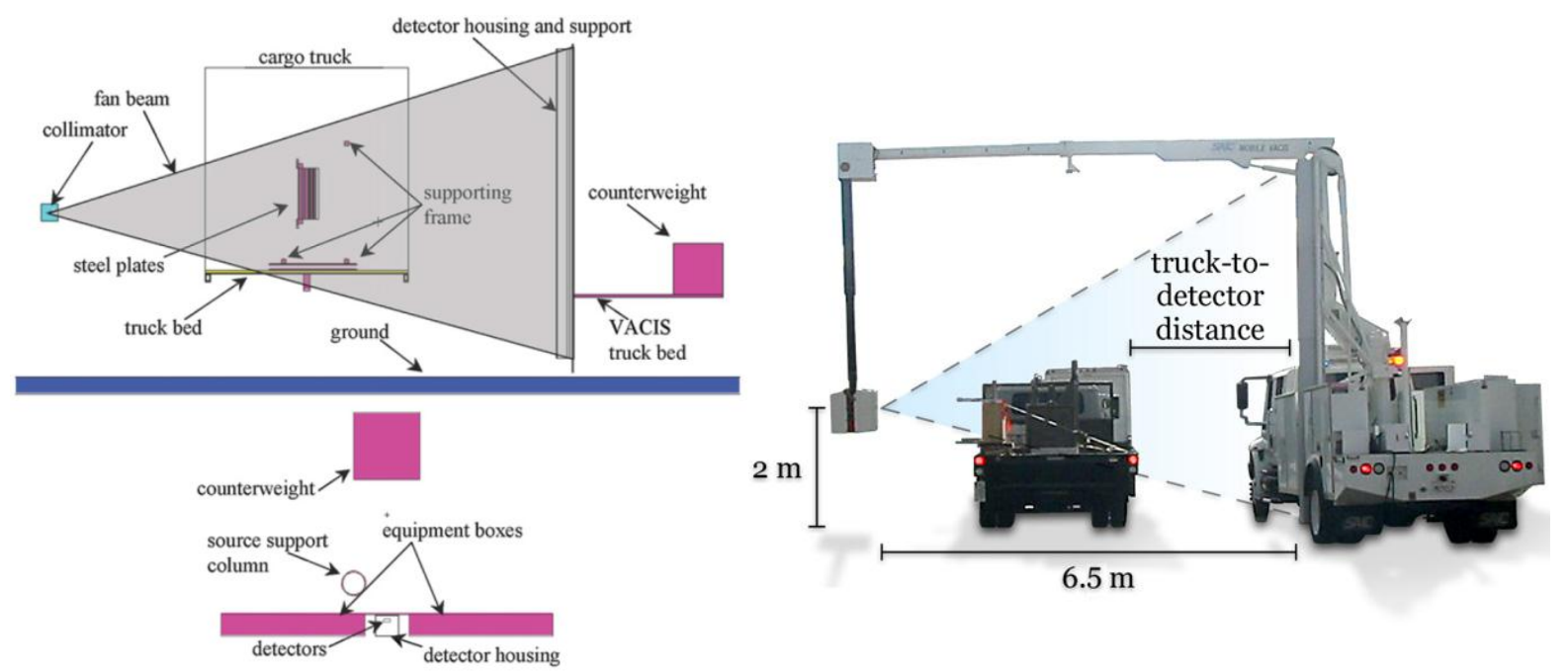

Figure 32. The geometry for the Radiography case. 


\subsection{Comparison of Results}

Figure 7.2 shows the MCNP5 run results of relative detector response as a function of height for a $17.8 \mathrm{~cm}(7-\mathrm{in})$ thick plate of steel. The runtime to simulate $3.4 \times 10^{7}$ histories is about 393 minutes. Note that the noise in the measured data is due to counting statistics, not any physical processes. The ADVANTG run yielded identical results to the analog MCNP run, but took about 639 minutes, or around $63 \%$ slower. The issues with the hybrid methodology is that point tallies, which are used for radiography cases essentially have infinite variance (poor performance when collisions are near the detector) and are themselves a form of variance reduction and one that is more suited to the problem than weight windows. The geometry itself is mostly air with a long mean free path and few thick obstacles.

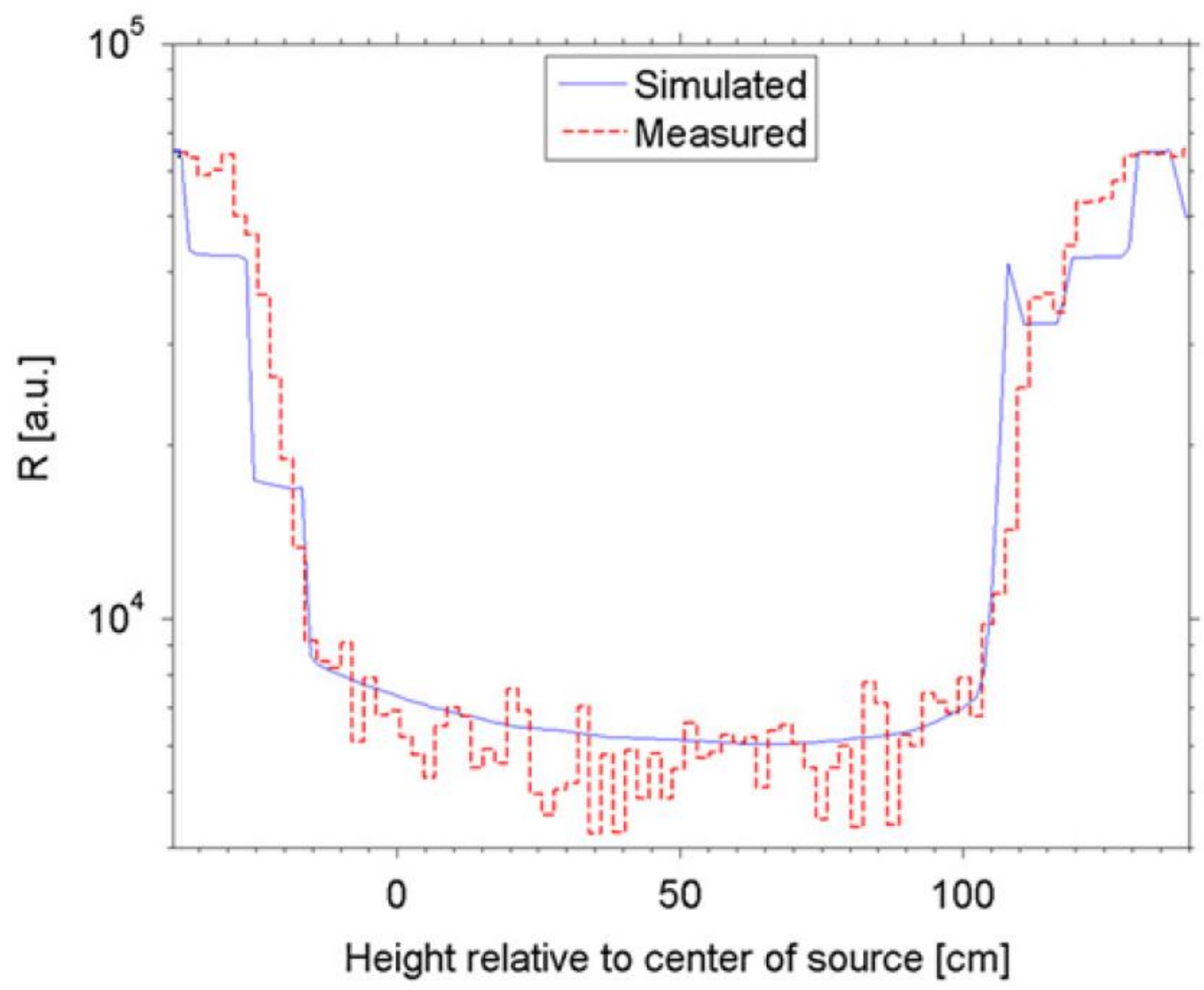

Figure 33. Measured and simulated response for the $17.8 \mathrm{~cm}$-thick case. 


\subsection{Polyethylene Coupled Neutron Gamma Case}

\subsection{Description}

Neutron-induced photon signatures are an important component of many radiation detection scenarios, but are computationally expensive. The purpose of the polyethylene coupled neutron gamma case is to define a basic well understood problem model with MCNP. The polyethylene coupled neutron photon problem uses a simple geometric configuration to demonstrate the simulation of a coupled neutron photon problem. A $8,000 \mathrm{~cm}^{3}$ cube of polyethylene is irradiated using a $40 \mathrm{mCi}{ }^{252} \mathrm{Cf}$ neutron source positioned $5 \mathrm{~cm}$ from one face of the polyethylene cube. $\mathrm{A}$ coaxial high-purity germanium (HPGe) spectrometer, with a relative efficiency of $11 \%$ (active crystal dimensions approximately $5 \mathrm{~cm}$ in diameter by $3 \mathrm{~cm}$ thick, full-width at half-maximum energy resolution of approximately $3 \mathrm{keV}$ at $1333 \mathrm{keV}$ ) is placed $5 \mathrm{~cm}$ away from the opposing face of the cube. Only the polyethylene cube with surrounding air is considered for neutron induced gamma production - zero gamma production in the detector is assumed. This is to allow comparisons with methods [Smith et al. 2009] which separate transport into a source region and a detector response region for the (n,y) problem [Burns 2009]. This case was done at PNNL, but funded by Gus Caffrey at INL. Figure 8.1 shows the problem geometry for this case.

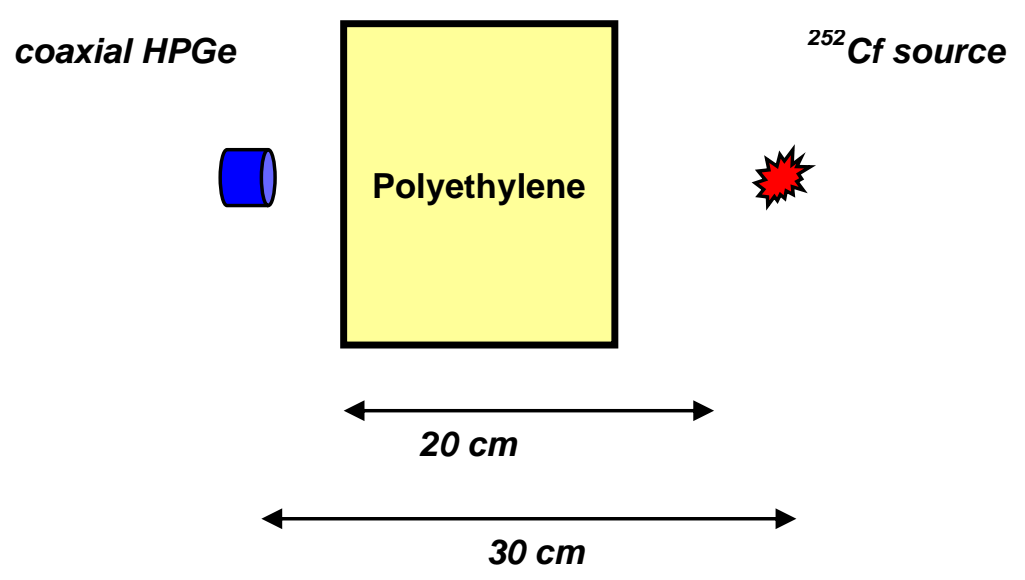

Figure 34. The geometry for the Polyethylene Coupled Neutron Gamma Case.

\subsection{Comparison of Results}

RADSAT was run with a global mesh size of 0.025 meters, Triangle Chebychev Legendre quadrature, S16P2 Galerkin scattering treatment and 79 neutron groups. Results for the pulseheight tally with HPGe spectrometer viewing the polyethylene sample are shown in Figure 8.2 assuming a 2 hour measurement time for a ${ }^{252} \mathrm{Cf}$ source with an activity of $40 \mathrm{mCi}$. Key observations from that comparison include the obscuring of the lower-energy, lower-yield 0.511 $\mathrm{MeV}$ annihilation line from the sample, but the enhancement of the double-escape peak at 1.20 $\mathrm{MeV}$ (due to relatively low stopping power, in the small HPGe spectrometer, for the $2.223 \mathrm{MeV}$ line produced from ${ }^{1} \mathrm{H}$ radiative capture). 
The ADVANTG results were essentially identical to the MCNP results and all of the peaks converged faster except for the $4.439 \mathrm{MeV}$ peak, which made the whole run slower. Part of the issue was that many histories were born outside of the weight windows. If one or two peaks were the only metrics of interest, ADVANTG could yield a speedup in the problem of around a factor of 20 , which would be substantial and take off about $95 \%$ of the run time, but still slower than the RADSAT run. Table 8.1 indicates the total count rate in the prominent peak regions for detector response.

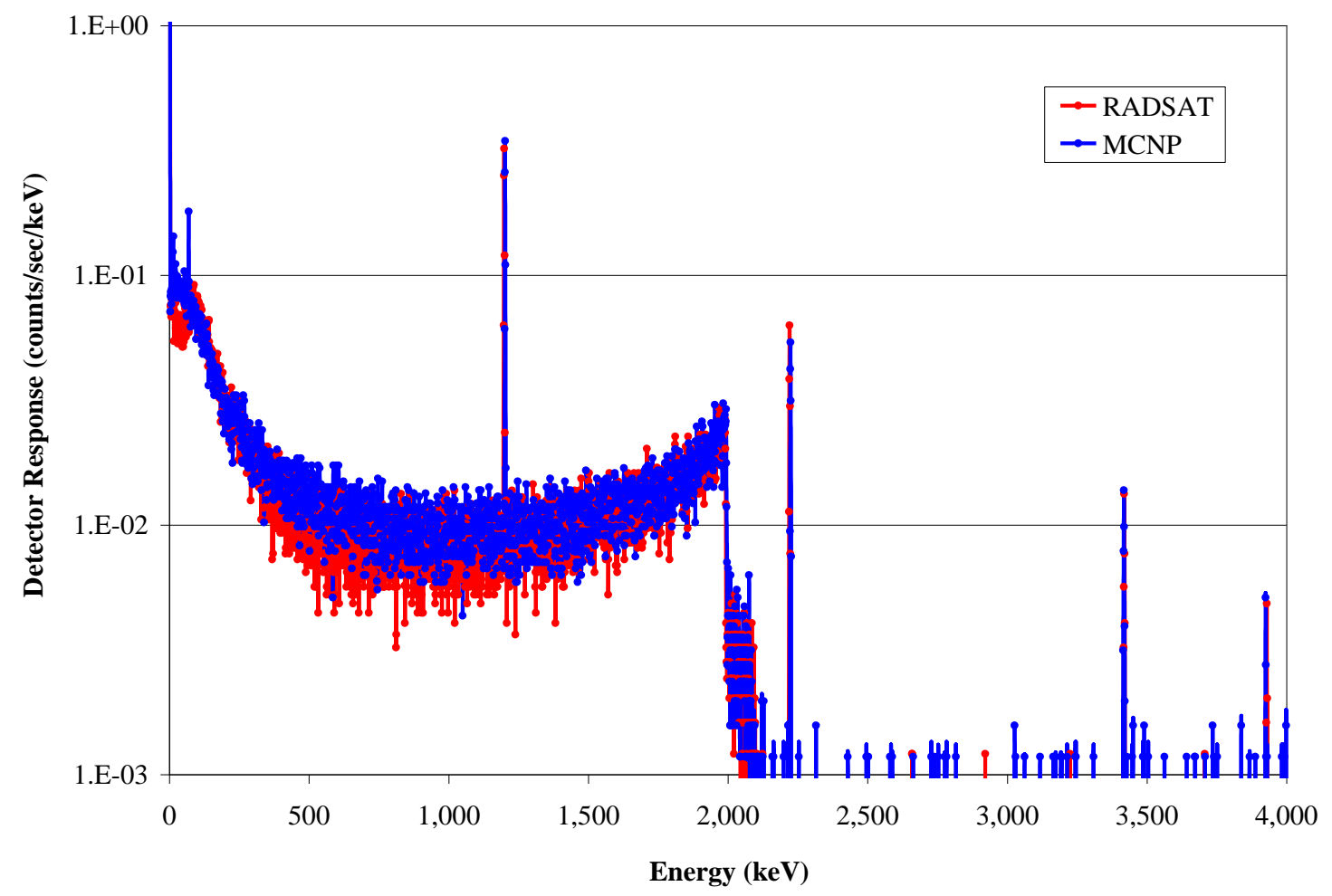

Figure 35. MCNP5 Results for Pulse-height Tally in HPGe Spectrometer

Table 8.1. Quantitative Peak Intensities for Polyethylene

\begin{tabular}{|c|c|c|c|c|c|c|c|}
\hline & \multicolumn{6}{|c|}{ Discrete Gamma-Ray Energy (MeV) } & Run Time \\
\hline Method & 1.2625 & 2.223 & 3.684 & 4.439 & 4.9465 & 6.25 & Hours \\
\hline $\begin{array}{c}\text { MCNP } \\
\left(y / \mathrm{sec}^{\left.-\mathrm{cm}^{2}\right)}\right.\end{array}$ & 2.88E-08 & 1.74E-05 & 2.99E-08 & $3.51 \mathrm{E}-07$ & 6.57E-08 & 2.54E-12 & 914 \\
\hline $\begin{array}{l}\text { ADVANTG } \\
\left(y / s^{\prime} c-c^{2}\right)\end{array}$ & 2.90E-08 & 1.75E-05 & 2.99E-08 & 3.52E-07 & $6.55 \mathrm{E}-08$ & $2.51 \mathrm{E}-12$ & $\begin{array}{l}936 \\
(46)\end{array}$ \\
\hline Diff (\%) & $0.82 \%$ & $0.36 \%$ & $0.15 \%$ & $0.41 \%$ & $0.23 \%$ & $1.07 \%$ & $\begin{array}{c}2.4 \% \\
(-95 \%)\end{array}$ \\
\hline $\begin{array}{c}\text { RADSAT } \\
\left(y / \text { sec-cm }^{2}\right)\end{array}$ & 2.88E-08 & 1.80E-05 & 3.09E-08 & 3.38E-07 & $6.70 \mathrm{E}-08$ & 3.47E-12 & 5 \\
\hline Diff (\%) & $0.05 \%$ & $3.44 \%$ & $3.23 \%$ & $3.82 \%$ & $1.91 \%$ & $36.6 \%$ & $-183,000 \%$ \\
\hline
\end{tabular}




\section{0 $\mathrm{UF}_{6}$ Cylinder Case}

\subsection{Description}

This case includes a model of $a \mathrm{UF}_{6}$ cylinder developed to determine the uranium enrichment from gamma-ray spectroscopy [Smith, et al. 2010]. It is an example of a multi-physics particle transport problem where various decay processes result in the detectable gamma emissions, as well as including a highly self-attenuating source volume. A more accurate prediction of enrichment is expected by modeling the contributions from each gamma emitting process, which are modeled a six separate and independent source terms to account for the main features of the measurement. In addition to the $185-\mathrm{keV}$ containing emission from ${ }^{235} \mathrm{U}$, the five additional processes include 1) gamma emission from ${ }^{238} \mathrm{U}$; 2) gamma emission from ${ }^{232} \mathrm{U} ; 3$ ) gammas produced in $(n, y)$ reactions from spontaneous fission neutron emission from $\left.{ }^{238} U ; 4\right)$ gammas produced in $(n, \gamma)$ reaction from neutrons resulting from ${ }^{19} \mathrm{~F}(\alpha, n)$ reactions induced by ${ }^{234} \mathrm{U}$ alpha decay; and 5) bremsstrahlung gammas produced from the beta decay of ${ }^{234 \mathrm{~m}} \mathrm{~Pa}$. The geometry is shown below in Figure 9.1. The detector system consists of three collimated 3"x3" Nal gamma detectors, shown below in Figure 9.2 with front and back polyethylene fences reflecting neutrons to iron around the detectors and the $30 \mathrm{~B}$ cylinder.
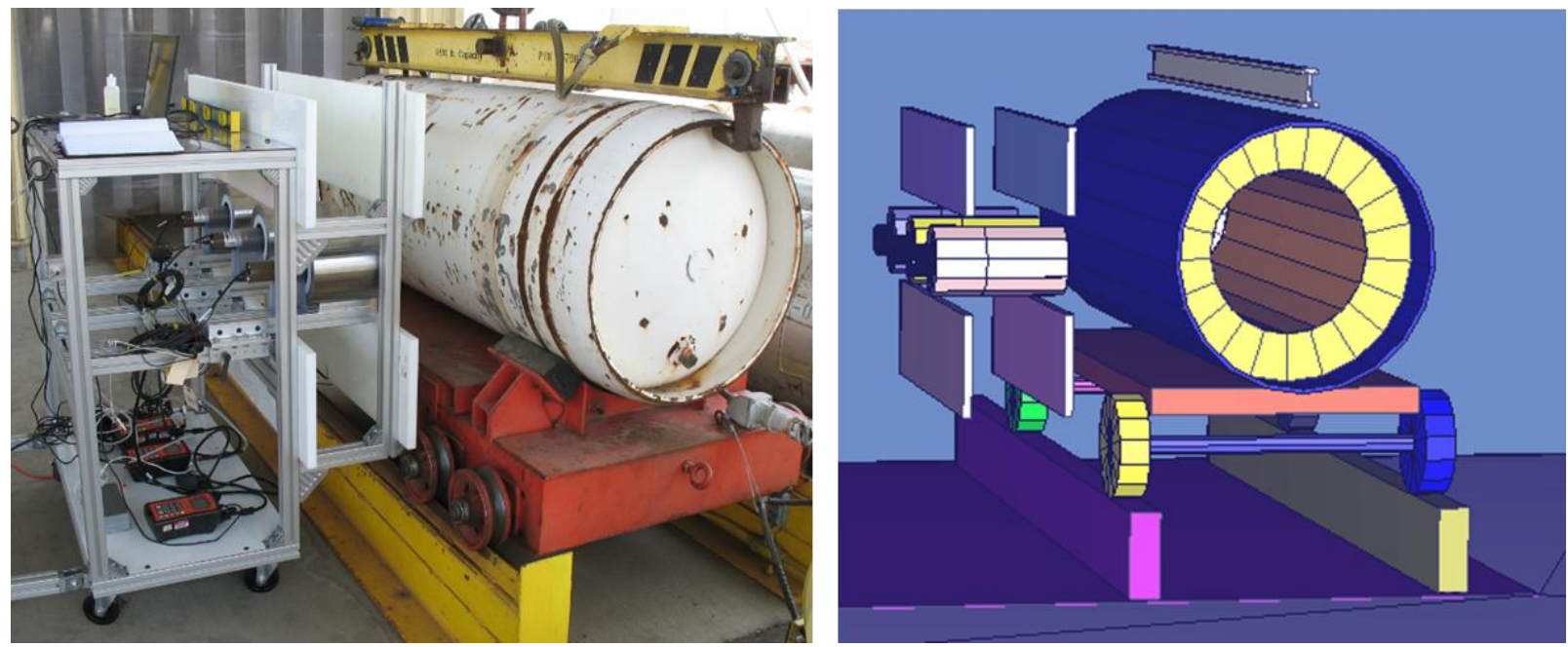

Figure 36. The geometry for the $\mathrm{UF}_{6}$ cylinder case (MCNP model geometry on the right).

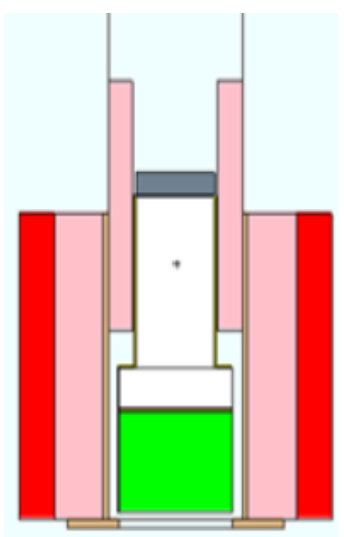

Figure 37. MCNP detector model geometry; (red) steel, (pink) poly and (green) Nal. 


\subsection{Comparison of Results}

Each of the individual sources mentioned above was run as an individual source term and calculation, and then the results are summed together in post-processing. Each of the MCNP cases were run with $10^{10}$ histories on a cluster of 128 processors. The runtimes are given below in Table 9.1. In addition to collimation and shielding, the iron serves as an $(n, y)$ converter to produce detectable $\sim 7.6-\mathrm{MeV}$ gammas. Therefore, the neutron source cases $\left[{ }^{238} \mathrm{U}\right.$ spontaneous fission and ${ }^{234} U$ decay induce $\left.(\alpha, n)\right]$ are run with both neutron and photon transport enabled. The spectra from the different sources and their sum are plotted below in Figure 9.3. The spectra are the summed response of three 3x3 Nal detectors for a UF6 30B cylinder with ${ }^{235} \mathrm{U}$ content of $4.0 \mathrm{wt} \%$. ADVANTG could not be used for this scenario because fission is not considered in fixed-source Denovo calculations and MCNP5 does not support an F8 tally with and coupled neutron gamma problem while using weight windows.

Table 9.1. MCNP Run Summary

\begin{tabular}{|l|c|c|c|}
\hline Sources & Transport & Histories & Run Time \\
\hline U235 & $\mathrm{V}$ & $10^{10}$ & $78 \mathrm{~m}$ \\
\hline U238 & $\mathrm{V}$ & $10^{10}$ & $78 \mathrm{~m}$ \\
\hline U232 & $\mathrm{V}$ & $10^{10}$ & $81 \mathrm{~m}$ \\
\hline U238sf & $\mathrm{n}, \mathrm{\gamma}$ & $10^{10}$ & $516 \mathrm{~m}$ \\
\hline U234a & $\mathrm{n}, \mathrm{\gamma}$ & $10^{10}$ & $496 \mathrm{~m}$ \\
\hline Pa234m & $\mathrm{Y}$ & $10^{10}$ & $78 \mathrm{~m}$ \\
\hline Total & $\mathrm{n}, \mathrm{\gamma}$ & $6 \times 10^{10}$ & $1,327 \mathrm{~m}(22 \mathrm{~h} \mathrm{7} \mathrm{min})$ \\
\hline
\end{tabular}

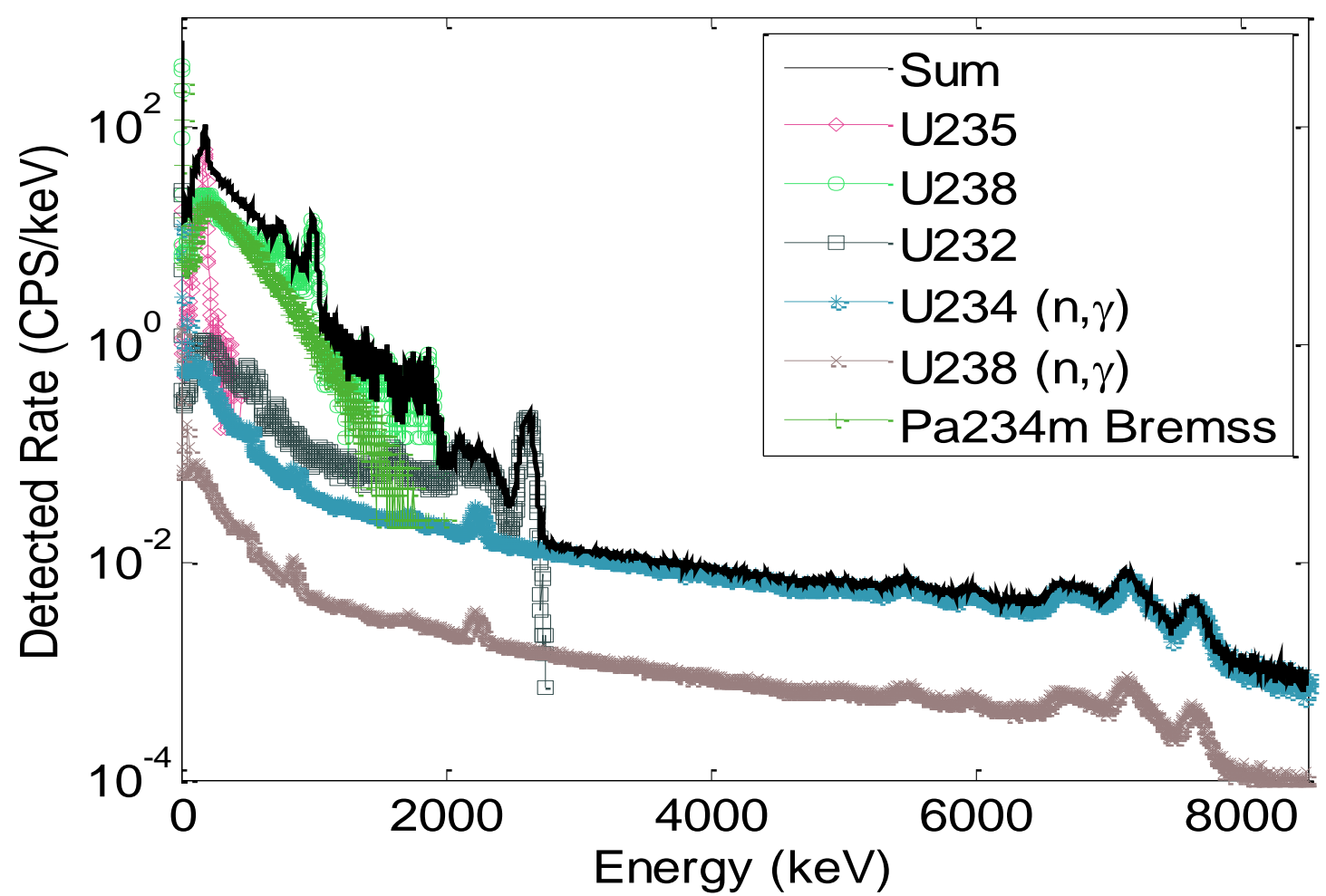

Figure 38. MCNP calculated detector response results. 
The percent difference between the measurement and MCNP5 result as a function of energy is also plotting in Figure 9.4. Because the sources in this case are varied and have many features, instead of peak or total counts, a residuals plot is analyzed to look at the agreement of the calculation over all energy groups. The large differences in the region between 1,100 and $2,000 \mathrm{keV}$ are from large uncertainty in the MCNP5 results for the ${ }^{238} \mathrm{U}$ vector, which has few emissions in this region. Similarly, the larger differences among the bins in the 3,000-8,500 keV range are to low counts in the measurement. However if we compare the total count rate in this range, which is used as the nontraditional enrichment metric, the results are quite similar (33.45 cps for MCNP5 and $32.25 \mathrm{cps}$ for the measurement). In regions where both MCNP5 and the measurement have low uncertainty, the percent difference in count rate is below $30 \%$.

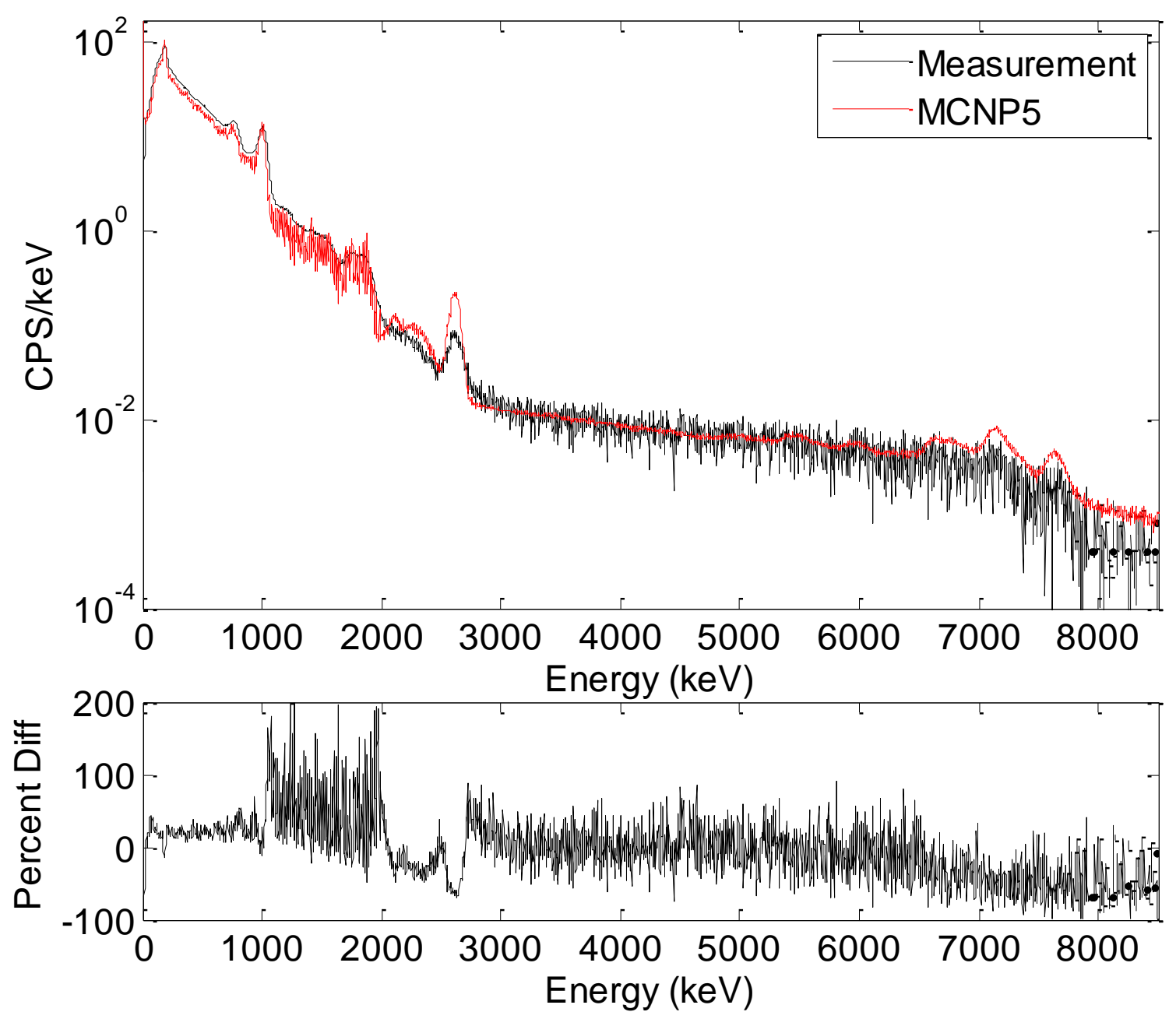

Figure 39. MCNP modeled versus measured data for a 4\% UF6 30B cylinder, with residuals plot on the bottom. 


\section{References}

Bell, G., S. Glasstone, “Nuclear Reactor Theory”, Van Nostrand, NY, 1970.

Burns, KA, Coupled Multi-Group Neutron Photon Transport for the Simulation of HighResolution Gamma-Ray Spectroscopy Application, Ph.D. Dissertation, Georgia Institute of Technology, 2009.

Burns K.A., L.E. Smith, C.J. Gesh, M.W. Shaver, "A Coupled Deterministic-Monte Carlo Approach for the Simulation of Prompt Gamma Neutron Activation Analysis," 2009 International Conference on Mathematics, Computational Methods and Reactor Physics, Saratoga Springs, New York, 2009.

Caffrey A.J., "Recent Test Results for the INL Spent-Fuel Cask Gamma-Ray Scanner," Presented at the IAEA US SP Annual Meeting Presented on May 19, 2009.

Casella A.M., C.J. Gesh, L.E Smith, "Use of Detector Response Functions and Deterministic Flux Calculations in Count Rate Predictions," Transactions of the American Nuclear Society, 100, 2009.

Duderstadt, J.J., L.J. Hamilton, “Nuclear Reactor Analysis”, John Wiley and Sons, Inc., NY, 1976.

Evans T.M., A.S. Stafford, K.T. Clarno, "Denovo - A New Three-Dimensional Parallel Discrete Ordinates Code in SCALE"Nuclear Technology, June 2010.

Ganapol, B.D. "Analytical Benchmarks for Nuclear Engineering Applications: Case Studies in Neutron Transport Theory". Department of Aerospace and Mechanical Engineering University of Arizona Nuclear Energy Agency Organisation for Economic Co-Operation and Development (OECD). NEA No. 6292, 2008.

Kernan WJ, EK Mace, ER Siciliano, KE Conlin, EL Flumerfelt, RT Kouzes, and ML Woodring. "Modeling and Measurements for Mitigating Interface from Skyshine." In IEEE Nuclear Science Symposium Conference Record (NSS/MIC 2009), pp. 976 - 980, 2009.

Lamarsh, J.R., "Introduction to Nuclear Engineering", Addison-Wesley, NY, 1983.

Lewis E. E. and W. F. Miller, "Computational Methods of Neutron Transport", John Wiley and Sons, New York, 1984.

McDonald B.S., D.V. Jordan, C.T. Orton, E.K. Mace, R.S. Wittman, and L.E. Smith, "Model and Algorithm Evaluation for the Hybrid UF6 Container Inspection System", Institute of Nuclear Materials Management Annual Meeting, 2011.

McGhee J, Wareing TA, Barnett DA. "Attila User's Manual”, Gig Harbor, WA, Transpire, Inc., 2007

Miller EA, Joseph A Caggiano, Robert C Runkle, Timothy A White, Aaron M Bevill, "Scatter in Cargo Radiography", Appl Radiat Isot Mar, 69 (3), 594-603, 2011. 
Mosher S.W., T.M. Evans, T.M. Miller, and J.C. Wagner, "Efficient Transport Simulations of Difficult Detection Problems Using ADVANTG," 2009 IEEE Nuclear Science Symposium and Medical Imaging Conference, Orlando, Florida, October, 2009.

Parringron, J. R. et al. "Nuclides and Isotopes Fifteenth Edition: Chart of the Nuclides". San Jose, CA: Lockheed Martin and General Electric Nuclear Energy, 1996.

Sandness GA, JE Schweppe, WK Hensley, MM Conrady, JD Borgardt, and AL Mitchell. "Accurate Modeling of the Gamma-Ray Background for Homeland Security Applications", PNNL-20411, Pacific Northwest National Laboratory, Richland, WA, 2011.

Shaver MW. "Radiation Detection Scenario Analysis Toolbox (RADSAT) Test Case Implementation Final Report”. PNNL-19795, Pacific Northwest National Laboratory, Richland, WA, 2010.

Shaver M. W., L. E. Smith, R. T. Pagh, E. A. Miller, R. S. Wittman, "The Coupling of a Deterministic Transport Field Solution to a Monte Carlo Boundary Condition for the Simulation of Large Gamma-Ray Spectrometers," Nuclear Technology, 168, 2009.

Smith H.P. and J.C. Wagner, "A Case Study in Manual and Automated Monte Carlo Variance Reduction with a Deep Penetration Reactor Shielding Problem," Nucl. Sci. Eng., 149, 23-37, 2005.

Smith L. E., J.E. Ellis, C.J. Gesh, R.J. McConn, G.H. Meriwether, E. Miller, R.T. Pagh, A.B. Valsan, T. Wareing, "Deterministic Transport Methods for the Simulation of Gamma-Ray Spectroscopy Scenarios," IEEE Transactions on Nuclear Science, 55, 2598-2606, 2008.

Smith, L.Eric, Christopher J. Gesh, Richard T. Pagh, Erin A. Miller, Mark W. Shaver, Eric D. Ashbaker, Michael T. Batdorf, J. Edward Ellis, William R. Kaye, Ronald J. McConn, George H. Meriwether, Jennifer J. Ressler, Andrei B. Valsan, and Todd A. Wareing, "Coupling Deterministic and Monte Carlo Transport Methods for the Simulation of Gamma-Ray Spectroscopy Scenarios", IEEE Transactions on Nuclear Science, Vol. 55, No. 5, 2598, 2008.

Smith, L. Eric, Emily K. Mace, Alex C. Misner, and Mark W. Shaver, "Signatures and Methods for the Automated Nondestructive Assay of UF6 Cylinders at Uranium Enrichment Plants", IEEE Transactions on Nuclear Science, Vol. 57, No. 4, 2247, 2010.

Stewart J.E., "Ch. 14: Principles of Total Neutron Counting, in Passive Nondestructive Assay of Nuclear Materials” Los Alamos National Laboratory, LA-UR-90-732, NUREG/CR-5550, 1991.

Wagner J. C. and A. Haghighat, "Automatic Variance Reduction of Monte Carlo Shielding Calculations Using the Discrete Ordinates Adjoint Function," Nucl. Sci. Eng., 128, 186-208, 1998.

Wagner J. C., "An Automated Deterministic Variance Reduction Generator for Monte Carlo Shielding Applications," Proceedings of the American Nuclear Society 12th Biennial RPSD Topical Meeting, Santa Fe, New Mexico, 2002. 
Wagner J. C., E. D. Blakeman, and D. E. Peplow, "Forward-Weighted CADIS Method for Variance Reduction of Monte Carlo Calculations of Distributions and Multiple Localized Quantities," Intl. Conf. Math. Comp. Meth. Reactor Phys. (M\&C 2009), Saratoga Springs, New York, 2009.

Waters, L.S., Editor, “MCNPX User's Manual”, Version 2.3.0, LA-UR-02-2607, Los Alamos National Laboratory, April 2002.

X-5 Monte Carlo Team, "MCNP - A General Purpose Monte Carlo N-Particle Transport Code, Version 5", LA-UR-03-1987, Los Alamos National Laboratory, April 2003. 


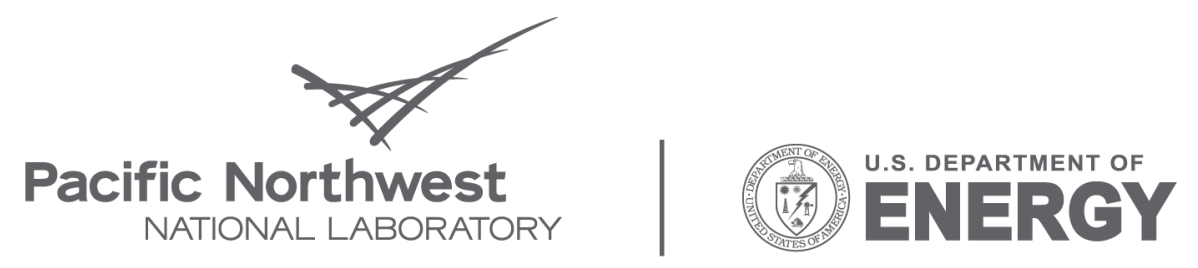

Proudly Operated by Battelle Since 1965

902 Battelle Boulevard

P.O. Box 999

Richland, WA 99352

1-888-375-PNNL (7665)

www.pnl.gov 